\title{
PERIODICITY IN DISTRIBUTION. I. DISCRETE SYSTEMS
}

\section{A. YA. DOROGOVTSEV}

Received 5 January 2001 and in revised form 1 October 2001

\begin{abstract}
We consider the existence of periodic in distribution solutions to the difference equations in a Banach space. A random process is called periodic in distribution if all its finitedimensional distributions are periodic with respect to shift of time with one period. Only averaged characteristics of a periodic process are periodic functions. The notion of the periodic in distribution process gave adequate description for many dynamic stochastic models in applications, in which dynamics of a system is obviously nonstationary. For example, the processes describing seasonal fluctuations, rotation under impact of daily changes, and so forth belong to this type. By now, a considerable number of mathematical papers has been devoted to periodic and almost periodic in distribution stochastic processes. We give a survey of the theory for certain classes of the linear difference equations in a Banach space. A feature of our treatment is the analysis of the solutions on the whole of axis. Such an analysis gives simple answers to the questions about solution stability of the Cauchy problem on $+\infty$, solution stability of analogous problem on $-\infty$, or of existence solution for boundary value problem and other questions about global behaviour of solutions. Examples are considered, and references to applications are given in remarks to appropriate theorems.
\end{abstract}

2000 Mathematics Subject Classification: 34-02, 34F05, 34K50, 60-02, 60H10, 37L55, 93E03.

1. Bounded and periodic solutions of difference equations. In this section, we construct an explicit representation of bounded or periodic solutions for abstract deterministic linear difference equation with a constant or periodic operator coefficient and bounded or periodic input. Then, general linear difference equations are studied too. The existence of bounded and periodic solutions is present for some difference equations with Lipschitz type nonlinearity and for the equation of Riccati type. Stability of solutions under bounded perturbation of operator coefficients is considered.

1.1. Notations. Let $(B,\|\cdot\|)$ be a complex Banach space with a zero element $\overline{0}$ and $\mathscr{L}(B)$ be a Banach algebra of all continuous linear mappings $A: B \rightarrow B$ with a unity element $I$ and zero element $\Theta$.

For an operator $A$ from $\mathscr{L}(B)$, let $\sigma(A)$ and $\rho(A)$ be the spectrum and resolvent set of $A$, respectively.

Suppose that $\sigma(A)$ is the union of two spectral sets $\sigma_{-}$and $\sigma_{+}$. If $\Gamma$ is a simple cycle which lies in $\mathbb{C} \backslash \sigma(A)$ and envelops $\sigma_{-}$, then the operators

$$
P_{-}:=-\frac{1}{2 \pi i} \oint_{\Gamma}(A-\lambda I)^{-1} d \lambda, \quad P_{+}:=I-P_{-}
$$


are well defined and belong to $\mathscr{L}(B)$ (see, e.g., [23] or [16]). Moreover, $P_{-}^{2}=P_{-}, P_{+}^{2}=P_{+}$, and $P_{-} P_{+}=P_{+} P_{-}=\Theta$. The operators $P_{-}$and $P_{+}$are called the spectral projectors or Riesz spectral projectors.

DEFINITION 1.1. The sequence $\{y(n): n \in \mathbb{Z}\}$ with $y(n) \in B, n \in \mathbb{Z}$ is bounded if

$$
\|y\|_{\infty}:=\sup _{n \in \mathbb{Z}}\|y(n)\|<+\infty .
$$

Definition 1.2. For fixed $p \in \mathbb{N}$, the sequence $\{y(n): n \in \mathbb{Z}\}$ with $y(n) \in B$, $n \in \mathbb{Z}$, is $p$-periodic if

$$
y(n+p)=y(n), \quad n \in \mathbb{Z} .
$$

Define

$$
S:=\{z \in \mathbb{C}:|z|=1\} .
$$

1.2. Simple linear equation. First, we give basic theorem about the difference equation with one operator coefficient. The many applications lead to the equation of such kind. The following result has been proved in [9]. Let $A \in \mathscr{L}(B)$ be a fixed operator.

THEOREM 1.3. The following statements are equivalent:

(a) the equation

$$
x(n+1)=A x(n)+y(n), \quad n \in \mathbb{Z}
$$

has a unique bounded solution $\{x(n): n \in \mathbb{Z}\}$ for every bounded sequence $\{y(n): n \in \mathbb{Z}\}$;

(b) for spectrum $\sigma(A)$, we have

$$
\sigma(A) \cap S=\varnothing .
$$

Proof. (a) implies (b). Let $\lambda_{0} \in S$ and $z \in B$. Let $\{x(n): n \in \mathbb{Z}\}$ be a unique bounded solution of (1.5) that corresponds to the bounded sequence $\left\{-\lambda_{0}^{n} z: n \in \mathbb{Z}\right\}$. Put

$$
u(n):=x(n) \lambda_{0}^{-n}, \quad n \in \mathbb{Z} .
$$

From formula (1.5), it is clear that the equation

$$
\lambda_{0} u(n+1)=A u(n)-z, \quad n \in \mathbb{Z}
$$

has a unique bounded solution $\{u(n): n \in \mathbb{Z}\}$ for every $z \in B$. Consequently,

$$
\lambda_{0}(u(n+1)-u(n))=A(u(n)-u(n-1)), \quad n \in \mathbb{Z} .
$$

Thus $u(n)=u(0)=: u$ for every $n \in \mathbb{Z}$. Therefore, given any element $z \in B$, there is a unique element $u \in B$ such that

$$
\left(A-\lambda_{0} I\right) u=z
$$

Thus, by the Banach theorem, the operator $A-\lambda_{0} I$ is invertible. 
(b) implies (a). Let

$$
\sigma_{-}:=\sigma(A) \cap\{z \in \mathbb{C}:|z|<1\}, \quad \sigma_{+}:=\sigma(A) \backslash \sigma_{-},
$$

and $P_{-}, P_{+}$are the corresponding spectral projectors. It is easy to show that

$$
L:=\sum_{j=0}^{\infty}\left\|\left(A P_{-}\right)^{j}\right\|+\sum_{j=-\infty}^{-1}\left\|\left(A P_{+}\right)^{j}\right\|<+\infty,
$$

where $\left(A P_{-}\right)^{0}:=P_{-}$(see, e.g., [16, 23]). If $\{y(n): n \in \mathbb{Z}\}$ is a bounded sequence in $B$, then the sequence

$$
x(n):=\sum_{j \in \mathbb{Z}} G(j) y(n-1-j), \quad n \in \mathbb{Z},
$$

where

$$
G(j):= \begin{cases}\left(A P_{-}\right)^{j}, & j \geq 0 ; \\ -\left(A P_{+}\right)^{j}, & j \leq-1, j \in \mathbb{Z}\end{cases}
$$

is also bounded. Moreover, for every $n \in \mathbb{Z}$, we have

$$
\begin{aligned}
A x(n) & =\left(A P_{-}+A P_{+}\right) x(n) \\
& =\sum_{j=0}^{\infty}\left(A P_{-}\right)^{j+1} y(n-1-j)-\sum_{j=-\infty}^{-1}\left(A P_{+}\right)^{j+1} y(n-1-j) \\
& =x(n+1)-P_{-} y(n)-P_{+} y(n)=x(n+1)-y(n) .
\end{aligned}
$$

Now, we prove that the solution $\{x(n): n \in \mathbb{Z}\}$ for (1.5) is unique. Let $\{u(n): n \in \mathbb{Z}\}$ be a solution of (1.5) which corresponds to $\{y(n): n \in \mathbb{Z}\}$. Then, the sequence

$$
\{v(n):=x(n)-u(n): n \in \mathbb{Z}\}
$$

satisfies the equation

$$
v(n+1)=A v(n), \quad n \in \mathbb{Z},
$$

which is equivalent to the system

$$
v_{-}(n+1)=\left(A P_{-}\right) v_{-}(n), \quad v_{+}(n+1)=\left(A P_{+}\right) v_{+}(n) ; \quad n \in \mathbb{Z}
$$

with

$$
v_{-}(n):=P_{-} v(n), \quad v_{+}(n):=P_{+} v(n), \quad n \in \mathbb{Z} .
$$

From this we have, for $m \geq 1$,

$$
\begin{aligned}
& \left\|v_{-}(n)\right\|=\left\|\left(A P_{-}\right)^{m} v_{-}(n-m)\right\| \leq\left\|\left(A P_{-}\right)^{m}\right\| \sup _{k \in \mathbb{Z}}\left\|v_{-}(k)\right\|, \\
& \left\|v_{+}(n)\right\|=\left\|\left(A P_{+}\right)^{-m} v_{+}(n+m)\right\| \leq\left\|\left(A P_{+}\right)^{-m}\right\| \sup _{k \in \mathbb{Z}}\left\|v_{+}(k)\right\| .
\end{aligned}
$$

Therefore, $v_{-}(n)=\overline{0}=v_{+}(n), n \in \mathbb{Z}$. 
Let $\left\{A_{0}, A_{1}\right\} \subset \mathscr{L}(B)$ and $\{x(n)\}:=\{x(n): n \in \mathbb{Z}\}$.

COROLLARY 1.4. If $A_{0} A_{1}=A_{1} A_{0}$ holds, then the following statements are equivalent:

(a) the equation

$$
x(n+1)=A_{0} x(n)+A_{1} x(n-1)+y(n), \quad n \in \mathbb{Z}
$$

has a unique bounded solution $\{x(n)\}$ for every bounded sequence $\{y(n)\}$;

(b) for every $\lambda \in S$, the operator $\lambda^{2} I-\lambda A_{0}-A_{1}$ has a bounded inverse.

Proof. (a) implies (b). The argument used to establish the first part of Theorem 1.3 can be adapted easily to get this result.

(b) implies (a). It is a direct consequence of Theorem 1.3 for the equation

$$
\left(\begin{array}{c}
x(n+1) \\
x(n)
\end{array}\right)=\left(\begin{array}{cc}
A_{0} & A_{1} \\
I & \Theta
\end{array}\right) \cdot\left(\begin{array}{c}
x(n) \\
x(n-1)
\end{array}\right)+\left(\begin{array}{cc}
I & \Theta \\
\Theta & \Theta
\end{array}\right) \cdot\left(\begin{array}{c}
y(n) \\
y(n-1)
\end{array}\right), \quad n \in \mathbb{Z} .
$$

1.3. Periodic solutions. We give the condition under which (1.5), with a $p$-periodic sequence $\{y(n): n \in \mathbb{Z}\}$, has a unique $p$-periodic solution. Put

$$
S_{p}:=\left\{z \in \mathbb{C}: z^{p}=1\right\} .
$$

THEOREM 1.5. The following statements are equivalent:

(a) equation (1.5) has a unique $p$-periodic solution $\{x(n)\}$ for every $p$-periodic input $\{y(n)\}$;

(b) for spectrum $\sigma(A)$, we have

$$
\sigma(A) \cap S_{p}=\varnothing .
$$

Proof. It is immediate. A complete proof of Theorem 1.5 can be found in [9, Chapter 1 , Section 1.1].

1.4. General linear equation with one operator coefficient. We now consider a generalization of (1.5). To this generalization lead also some applications (see, e.g., [26, 32, 35]). We need the following condition.

Condition 1.6. Let $\omega$ be a complex-valued function which is analytic in some neighbourhood of circle $S$.

Under Condition 1.6, the function $\omega$ may be written as the Laurent series:

$$
\omega(z)=\sum_{n \in \mathbb{Z}} a_{n} z^{n}, \quad n \in \mathbb{Z},
$$

where

$$
\begin{aligned}
a_{n} & =\frac{1}{2 \pi i} \oint_{S} \omega(z) z^{-n-1} d z, \quad n \in \mathbb{Z} ; \\
r & =\limsup _{n \rightarrow-\infty}\left|a_{n}\right|^{1 /|n|}, \quad R=\left(\limsup _{n \rightarrow+\infty}\left|a_{n}\right|^{1 / n}\right)^{-1} .
\end{aligned}
$$

By Condition 1.6, we have $r<1<R$. 
Let $A$ be a closed linear operator with dense domain $D(A)$ in $B$. We consider the equation

$$
\sum_{k \in \mathbb{Z}} a_{k} x(n+k)=A x(n)+y(n), \quad n \in \mathbb{Z},
$$

where $\{y(n)\}$ is a bounded sequence in $B$.

DEFINITION 1.7. Sequence $\{x(n)\}$ is called a solution of (1.27), if

(i) for all $n \in \mathbb{Z}, x(n) \in D(A)$;

(ii) sequence $\{x(n)\}$ satisfies (1.27).

Let $\mathcal{M}$ be the class of all sequences $\{c(n)\} \subset \mathscr{L}(B)$ satisfying the conditions:

(i) for all $n \in \mathbb{Z}, c(n): B \rightarrow D(A)$;

(ii) for all $n \in \mathbb{Z}, A c(n) \in \mathscr{L}(B)$; $A c(n)=c(n) A$ on $D(A)$;

(iii) $\limsup _{n \rightarrow \infty}\|c(n)\|^{1 / n}<1, \limsup _{n \rightarrow-\infty}\|c(n)\|^{1 /|n|}<1 ; \limsup _{n \rightarrow \infty}\|A c(n)\|^{1 / n}$ $<1, \limsup _{n \rightarrow-\infty}\|A c(n)\|^{1 /|n|}<1$.

THEOREM 1.8. If (1.27) has a unique bounded solution $\{x(n)\}$ for every bounded sequence $\{y(n)\}$, then

$$
\sigma(A) \cap \omega(S)=\varnothing .
$$

If an operator A satisfies condition (1.28), then (1.27) has a unique bounded solution $\{x(n)\}$ for every bounded sequence $\{y(n)\}$; and there exists a sequence $\{c(n)\} \in M$ such that,

$$
x(n)=\sum_{k \in \mathbb{Z}} c(k-n) y(k), \quad n \in \mathbb{Z} .
$$

Proof. (1) Suppose that (1.27) has a unique bounded solution $\{x(n)\}$ for every bounded input $\{y(n)\}$. Let $v \in B, \lambda_{0} \in S$. For a unique bounded solution $\{x(n)\}$ of (1.27) with

$$
\left\{y(n)=-\lambda_{0}^{n} v: n \in \mathbb{Z}\right\},
$$

we have

$$
\sum_{k \in \mathbb{Z}} a_{k} \lambda_{0}^{k} u(n+k)=A u(n)-v
$$

where $u(n):=x(n) \lambda_{0}^{-n} \in D(A), n \in \mathbb{Z}$. The bounded solution $\{u(n)\}$ of (1.31) is also unique. Therefore,

$$
\sum_{k \in \mathbb{Z}} a_{k} \lambda_{0}^{k}[u(n+k)-u(n-1+k)]=A[u(n)-u(n-1)], \quad n \in \mathbb{Z} ;
$$

and by uniqueness of solution for (1.31), we conclude that

$$
\exists u \in D(A): u(n)=u(0)=u, \quad n \in \mathbb{Z} .
$$

Thus

$$
\forall v \in B, \quad \exists ! u \in D(A):\left(A-\omega\left(\lambda_{0}\right) I\right) u=v ;
$$

and by the closed graph theorem, it follows that operator $\left(A-\omega\left(\lambda_{0}\right) I\right)^{-1}$ is continuous. 
(2) From assumption (1.28) and the closedness of the set $\sigma(A)$, it follows that there exists an annulus

$$
K:=\left\{z \in \mathbb{C}: t_{0}<|z|<t_{1}\right\},
$$

with some $r<t_{0}<1<t_{1}<R$ such that

$$
\sigma(A) \cap \omega(K)=\varnothing .
$$

For the operator-valued function

$$
\Phi(z):=(\omega(z) I-A)^{-1}, \quad z \in K,
$$

we have, for every $z \in K$,

$$
\Phi(z) \in \mathscr{L}(B), \quad \Phi(z): B \longrightarrow D(A)
$$

and the Laurent series

$$
\Phi(z)=\sum_{n \in \mathbb{Z}} c(n) z^{n}, \quad z \in K
$$

where

$$
c(n)=\frac{1}{2 \pi i} \oint_{S} \Phi(z) z^{-n-1} d z, \quad n \in \mathbb{Z},
$$

see [23]. From the definition of the function $\Phi$, we have, for every $z \in K$ on $B$

$$
(\omega(z) I-A) \Phi(z)=I,
$$

or

$$
A \Phi(z)=\omega(z) \Phi(z)-I .
$$

Therefore, the function $A \Phi$ is analytic for $z \in K$ and, by closedness of the operator $A$, we deduce that

$$
A c(n)=\frac{1}{2 \pi i} \oint_{S} A \Phi(z) z^{-n-1} d z, \quad n \in \mathbb{Z},
$$

From (1.42), it follows that

$$
\begin{aligned}
\operatorname{Ac}(n) & =\frac{1}{2 \pi i} \oint_{S}(\omega(\lambda) \Phi(\lambda)-I) \lambda^{-n-1} d \lambda \\
& =\frac{1}{2 \pi i} \oint_{S} \sum_{k \in \mathbb{Z}} \sum_{m \in \mathbb{Z}} a_{k} c(m) \lambda^{k+m-n-1} d \lambda-I \delta_{n, 0} \\
& =\sum_{k \in \mathbb{Z}} a_{k} c(n-k)-I \delta_{n, 0}, \quad n \in \mathbb{Z} .
\end{aligned}
$$

Thus, $\{c(n)\} \in \mathcal{M}$, condition (ii) of the definition of class $\mathcal{M}$ is a consequence of the equality

$$
A \Phi(z)=\omega(z) \Phi(z)-I=\Phi(z) A, \quad z \in K
$$

on $D(A)$. 
For a bounded sequence $\{y(n)\}$, set

$$
x(n):=\sum_{k \in \mathbb{Z}} c(k-n) y(k), \quad n \in \mathbb{Z} .
$$

It is clear that

$$
\|x\|_{\infty} \leq \sum_{k \in \mathbb{Z}}\|c(k)\| \cdot\|y\|_{\infty}
$$

The closedness of $A$ implies that $x(n) \in D(A)$ for $n \in \mathbb{Z}$, and

$$
A x(n)=\sum_{k \in \mathbb{Z}} A c(k-n) y(k), \quad n \in \mathbb{Z} .
$$

From (1.48) and (1.44), it follows that

$$
\begin{aligned}
\sum_{k \in \mathbb{Z}} a_{k} x(n+k)-A x(n) & =\sum_{k \in \mathbb{Z}} a_{k} \sum_{m \in \mathbb{Z}} c(m-n-k) y(m)-\sum_{m \in \mathbb{Z}} A c(m-n) y(m) \\
& =\sum_{m \in \mathbb{Z}}\left(\sum_{k \in \mathbb{Z}} a_{k} c(m-n-k)-A c(m-n)\right) y(m) \\
& =\sum_{m \in \mathbb{Z}} I \delta_{m-n, 0} y(m)=y(n), \quad n \in \mathbb{Z} .
\end{aligned}
$$

Thus $\{x(n)\}$ is a bounded solution of (1.27).

To conclude the proof, we have to show that solution $\{x(n)\}$ is unique. Let $\{u(n)\}$ be a bounded solution of (1.27). Then the sequence $\{v(n):=x(n)-u(n)\}$ is a bounded solution of the equation

$$
\sum_{k \in \mathbb{Z}} a_{k} v(n+k)=A v(n), \quad n \in \mathbb{Z} .
$$

Let $\{w(n)\}$ be a bounded solution of (1.27) with $y(n)=v(n), n \in \mathbb{Z}$, such that

$$
w(n)=\sum_{k \in \mathbb{Z}} c(k-n) v(k), \quad n \in \mathbb{Z}
$$

where $\{c(n)\} \in \mathcal{M}$. Using (1.50), we obtain

$$
\begin{aligned}
v(n) & =\sum_{k \in \mathbb{Z}} a_{k} w(n+k)-A w(n) \\
& =\sum_{k \in \mathbb{Z}} a_{k} \sum_{m \in \mathbb{Z}} c(m-n-k) v(m)-A \sum_{m \in \mathbb{Z}} c(m-n) v(m) \\
& =\sum_{k \in \mathbb{Z}} a_{k} \sum_{m \in \mathbb{Z}} c(m) v(n+k+m)-A \sum_{m \in \mathbb{Z}} c(m) v(n+m) \\
& =\sum_{m \in \mathbb{Z}} c(m)\left(\sum_{k \in \mathbb{Z}} a_{k} v(n+k+m)-A v(n+m)\right)=\overline{0} .
\end{aligned}
$$


1.4.1. Examples. We want to illustrate by means of simple examples how Theorem 1.8 may be applied.

(a) It is easy to see that the existence of Theorem 1.3 is a simple consequence of Theorem 1.8.

(b) The equation

$$
x(n+1)-2 x(n)+x(n-1)=A x(n)+y(n), \quad n \in \mathbb{Z}
$$

has a unique bounded solution for every bounded input $\{y(n)\}$, if and only if

$$
\sigma(A) \cap[-4,0]=\varnothing .
$$

(c) The equation

$$
\frac{1}{2}(x(n+1)-x(n-1))=A x(n)+y(n), \quad n \in \mathbb{Z}
$$

has a unique bounded solution for every bounded input $\{y(n)\}$, if and only if

$$
\sigma(A) \cap\{\text { is } \mid s \in[-1,1]\}=\varnothing .
$$

(d) For (1.5), we have from Theorem 1.8 the generalization of Theorem 1.3 to the unbounded operator $A$.

REMARK 1.9. The particular cases of Theorem 1.8 can be improved in different ways [9].

Theorem 1.8 can be generalized in the following way.

Condition 1.10. Let $\{U(n): n \in \mathbb{Z}\} \subset \mathscr{L}(B)$ be a sequence of operators such that

$$
\limsup _{n \rightarrow-\infty}\|U(n)\|^{1 /|n|}<1, \quad \limsup _{n \rightarrow \infty}\|U(n)\|^{1 / n}<1 .
$$

Define

$$
\Omega(z):=\sum_{n \in \mathbb{Z}} U(n) z^{n}
$$

The operator-valued function $\Omega$ is defined by Condition 1.10 in some neighbourhood of the circle $S$.

THEOREM 1.11. The following statements are equivalent:

(i) the equation

$$
\sum_{k \in \mathbb{Z}} U(k) x(n+k)=A x(n)+y(n), \quad n \in \mathbb{Z}
$$

has a unique bounded solution $\{x(n)\}$ for every bounded sequence $\{y(n)\}$;

(ii) for every $z \in S$, the operator

$$
A-\Omega(z)
$$

has a continuous inverse.

Proof. The argument used to establish Theorem 1.8 can be adapted to get this result. 
1.5. Equation with periodic coefficients. Let $p \in \mathbb{N}$ and $\{A(n): n \in \mathbb{Z}\} \subset \mathscr{L}(B)$ be a sequence of bounded operators such that

$$
A(n+p)=A(n), \quad n \in \mathbb{Z} .
$$

Now, we first consider the equation

$$
x(n+1)=A(n) x(n)+y(n), \quad n \in \mathbb{Z},
$$

where $\{y(n)\}$ is a bounded sequence in $B$. It is a simple situation in which the previous results can be applied. Put

$$
D:=A(p-1) A(p-2) \cdots A(1) A(0)
$$

THEOREM 1.12 (see [14]). The following statements are equivalent:

(i) equation (1.62) has a unique bounded solution $\{x(n)\}$ for every bounded sequence $\{y(n)\}$;

(ii) for spectrum $\sigma(D)$, we have

$$
\sigma(D) \cap S=\varnothing
$$

Proof. (i) implies (ii). Let $\{x(n)\}$ be a unique solution of (1.62) for a bounded sequence $\{y(n)\}$. Iterating (1.62), we find that

$$
x((n+1) p)=D x(n p)+z(n)
$$

where

$$
z(n):=\sum_{j=0}^{p-2} A(p-1) \cdots A(j+1) y(n p+j)+y(n p+p-1) .
$$

The following statements hold:

(1) sequence $\{z(n)\}$ is bounded;

(2) for every bounded sequence $\{z(n)\}$ there exists a bounded sequence $\{y(n)\}$ such that equality (1.66) holds;

(3) the uniqueness of the solution (1.62) is equivalent to that of solution of (1.65). Therefore, if (1.62) has a unique bounded solution $\{x(n)\}$ for every bounded sequence $\{y(n)\}$, then the equation

$$
u(n+1)=D u(n)+z(n), \quad n \in \mathbb{Z}
$$

has a unique bounded solution $\{u(n)\}$ for every bounded sequence $\{z(n)\}$. By use of Theorem 1.3, we have (1.64).

(ii) implies (i). Given a bounded sequence $\{y(n)\}$, we define the bounded sequence $\{z(n)\}$ by (1.66). For sequence $\{z(n)\}$, by Theorem 1.3, equation (1.67) has a unique 
bounded solution $\{u(n)\}$. Sequence $\{x(n)\}$, defined by

$$
\begin{aligned}
x(n p):= & u(n) ; \\
x(n p+1):= & A(0) u(n)+y(n p) ; \\
x(n p+k):= & A(k-1) \cdots A(0) u(n) \\
& +\sum_{j=0}^{k-2} A(k-1) \cdots A(j+1) y(n p+j)+y(n p+k-1), \quad 2 \leq k \leq p-1, n \in \mathbb{Z},
\end{aligned}
$$

is bounded. This sequence $\{x(n)\}$ is a solution of (1.62) since

$$
\begin{aligned}
A(n p) x(n p) & =A(0) x(n p) \\
& =x(n p+1)-y(n p) ; \\
A(n p+1) x(n p+1) & =A(1) x(n p+1) \\
& =x(n p+2)-y(n p+1) ; \\
A(n p+k) x(n p+k) & =A(k) x(n p+k) \\
& =x(n p+k+1)-y(n p+k), \quad 1 \leq k \leq p-1 .
\end{aligned}
$$

The uniqueness is immediate.

REMARK 1.13. Condition (1.64) is equivalent, for every $k \in\{1,2, \ldots, p-1\}$, to the following equality:

$$
\sigma(A(k-1) \cdots A(0) A(p-1) A(p-2) \cdots A(k)) \cap S=\varnothing .
$$

This follows from the relation

$$
\sigma(A C) \backslash\{0\}=\sigma(C A) \backslash\{0\}
$$

which holds for every $\{A, C\} \subset \mathscr{L}(B)$. Indeed, if $z \neq 0$ and operator $A C-z I$ has an inverse $E$, then operator $z^{-1}(C E A-I)$ is inverse to $C A-z I$.

Let a function $\omega$ satisfy Condition 1.6 and $\left\{a_{k}: k \in \mathbb{Z}\right\}$ be its Laurent series coefficients. We now consider the equation

$$
\sum_{k \in \mathbb{Z}} a_{k} x(n+k)=A(n) x(n)+y(n), \quad n \in \mathbb{Z}
$$

for a bounded sequence $\{y(n)\}$ in $B$. Equation (1.72) can be rewritten as an equation from Theorem 1.12 by the following way. Let $B^{p}$ be a Banach space of columnvectors $\vec{u}$ with coordinates $u(1), u(2), \ldots, u(p)$ from $B$ and with norm

$$
\|\vec{u}\|:=\max _{1 \leq k \leq p}\|u(k)\| .
$$

Denote by

$$
\begin{aligned}
& \vec{x}(n):=(x(n p), x(n p+1), \ldots, x(n p+p-1))^{\prime}, \\
& \vec{y}(n):=(y(n p), y(n p+1), \ldots, y(n p+p-1))^{\prime},
\end{aligned}
$$


where "'” means the transition to column-vector. Let

$$
\begin{aligned}
\mathbb{A} & :=\left(\begin{array}{ccccc}
A(0) & \Theta & \Theta & \cdots & \Theta \\
\Theta & A(1) & \Theta & \cdots & \Theta \\
\Theta & \Theta & A(2) & \cdots & \Theta \\
\vdots & \vdots & \vdots & \ddots & \vdots \\
\Theta & \Theta & \Theta & \cdots & A(p-1)
\end{array}\right), \\
U(j):= & \left(\begin{array}{ccccc}
I a_{j p} & I a_{j p+1} & I a_{j p+2} & \cdots & I a_{j p+p-1} \\
I a_{j p-1} & I a_{j p} & I a_{j p+1} & \cdots & I a_{j p+p-2} \\
I a_{j p-2} & I a_{j p-1} & I a_{j p} & \cdots & I a_{j p+p-3} \\
\vdots & \vdots & \vdots & \ddots & \vdots \\
I a_{j p-p+1} & I a_{j p-p+2} & I a_{j p-p+3} & \cdots & I a_{j p}
\end{array}\right)
\end{aligned}
$$

for $j \in \mathbb{Z}$. Then $\{\mathbb{A}, U(j)\} \subset \mathscr{L}\left(B^{p}\right)$. It is a direct matter to verify that the problem of existence of a bounded solution for (1.72) in $B$ is equivalent to the problem of existence of a bounded solution for the equation

$$
\sum_{j \in \mathbb{Z}} U(j) \vec{x}(n+j)=\mathbb{A} \vec{x}(n)+\vec{y}(n), \quad n \in \mathbb{Z}
$$

in $B^{p}$

LEMMA 1.14. If Condition 1.6 for function $\omega$ holds, then

$$
\limsup _{n \rightarrow-\infty}\|U(n)\|^{1 /|n|}<1, \quad \limsup _{n \rightarrow \infty}\|U(n)\|^{1 / n}<1 .
$$

Proof. This result can be established by direct calculation.

Put

$$
\Phi_{j}(z):=I \sum_{n \in \mathbb{Z}} a_{n p+j} z^{n}, \quad 0 \leq j \leq p-1 .
$$

LEMMA 1.15. Given $z$ of some neighbourhood of $S$, the following equalities hold

$$
\Omega(z)=\sum_{n \in \mathbb{Z}} U(n) z^{n}=\left(\begin{array}{ccccc}
\Phi_{0}(z) & \Phi_{1}(z) & \Phi_{2}(z) & \cdots & \Phi_{p-1}(z) \\
z \Phi_{p-1}(z) & \Phi_{0}(z) & \Phi_{1}(z) & \cdots & \Phi_{p-2}(z) \\
z \Phi_{p-2}(z) & z \Phi_{p-1}(z) & \Phi_{0}(z) & \cdots & \Phi_{p-3}(z) \\
\vdots & \vdots & \vdots & \ddots & \vdots \\
z \Phi_{1}(z) & z \Phi_{2}(z) & z \Phi_{3}(z) & \cdots & \Phi_{0}(z)
\end{array}\right) .
$$

Proof. This is immediate.

LEMMA 1.16. Equation (1.72) has a unique bounded solution for every bounded sequence $\{y(n)\}$ if and only if the operator

$$
\mathbb{A}-\Omega(z)
$$

has a continuous inverse for every $z \in S$. 
Proof. This is a direct consequence of Theorem 1.12 and Lemmas 1.14 and 1.15.

Lemma 1.16 is not the final result for (1.72), since the analysis of spectrum of operator $\mathbb{A}-\Omega(z)$ is not simple even for particular cases.

EXERCISE. Characterize the bounded operators $A, C$ such that the equation

$$
x(n+1)=(A+C \sin n) x(n)+y(n), \quad n \in \mathbb{Z}
$$

has a unique bounded solution $\{x(n): n \in \mathbb{Z}\}$ for every bounded sequence $\{y(n)$ : $n \in \mathbb{Z}\}$.

1.6. Nonlinear equation. We now consider an example of nonlinear difference equation which satisfied the Lipschitz condition nonlinearity. This equation is connected with (1.27).

THEOREM 1.17. Let $A$ be a closed linear operator with dense domain $D(A)$ in $B$ and a function $\omega$ satisfying Condition 1.6 with Laurent series coefficients $\left\{a_{k}\right\}$. Let the sequence $\{c(n)\} \in \mathcal{M}$ corresponds to the operator $A$ and the function $\omega$ in Theorem 1.8. If $f: B \rightarrow B$ such that

$$
\|f(u)-f(v)\| \leq L\|u-v\|, \quad\{u, v\} \subset B
$$

with $L>0$, and

$$
L \max \left(\sum_{n \in \mathbb{Z}}\|c(n)\|, \sum_{n \in \mathbb{Z}}\|A c(n)\|\right)<1, \quad \sigma(A) \cap \omega(S)=\varnothing,
$$

then the equation

$$
\sum_{k \in \mathbb{Z}} a_{k} x(n+k)=A x(n)+f(x(n))+y(n), \quad n \in \mathbb{Z}
$$

has a unique bounded solution for every bounded sequence $\{y(n)\}$.

Proof. Let $\{y(n)\}$ be a bounded sequence in $B$. Consider the following successive approximations:

$$
x_{0}(n)=\overline{0}, \quad n \in \mathbb{Z}
$$

and for $m \geq 0$

$$
\sum_{k \in \mathbb{Z}} a_{k} x_{m+1}(n+k)=A x_{m+1}(n)+f\left(x_{m}(n)\right)+y(n), \quad n \in \mathbb{Z} .
$$

By Theorem 1.8, for every $m \geq 0,(1.86)$ has a unique bounded solution $\left\{x_{m}(n)\right\} \subset$ $D(A)$ and

$$
x_{m+1}(n)=\sum_{k \in \mathbb{Z}} c(k-n)\left(f\left(x_{m}(k)\right)+y(n)\right), \quad n \in \mathbb{Z}, m \geq 0 .
$$


By induction, it is easy to see that, for $m \geq 1$,

$$
\left\|x_{m+1}(n)-x_{m}(n)\right\| \leq L q_{1}\left\|x_{m}-x_{m-1}\right\|_{\infty} \leq\left(L q_{1}\right)^{m}\left(\|f(\overline{0})\|+\|y\|_{\infty}\right),
$$

where

$$
q_{1}:=\sum_{k \in \mathbb{Z}}\|c(k)\|
$$

Therefore, for every $n \in \mathbb{Z}$, there is an element $x(n) \in B$ such that

$$
x_{m}(n) \longrightarrow x(n), \quad n \rightarrow \infty
$$

in norm topology. By the closedness of $A$, we have

$$
A x_{m+1}(n)=\sum_{k \in \mathbb{Z}} A c(k-n)\left(f\left(x_{m}(k)\right)+y(k)\right), \quad n \in \mathbb{Z}, m \geq 1 .
$$

This implies the following:

$$
\left\|A x_{m+1}(n)-A x_{m}(n)\right\| \leq\left(L q_{2}\right)^{m}\left(\|f(\overline{0})\|+\|y\|_{\infty}\right), \quad m \geq 1
$$

for every $n \in \mathbb{Z}$ and

$$
q_{2}:=\sum_{k \in \mathbb{Z}}\|A c(k)\|
$$

Hence, for every $n \in \mathbb{Z}$, there is an element $u(n) \in B$ such that

$$
A x_{m}(n) \longrightarrow u(n), \quad m \longrightarrow \infty
$$

Then the closedness of $A$ implies that

$$
\forall n \in \mathbb{Z}: x(n) \in D(A), \quad A x(n)=u(n) .
$$

It is clear that the sequence $\{x(n)\}$ is bounded. We can apply (1.87) and (1.91) for $m \rightarrow \infty$ to obtain

$$
\begin{gathered}
x(n)=\sum_{k \in \mathbb{Z}} c(k-n)(f(x(k))+y(k)), \quad n \in \mathbb{Z} ; \\
A x(n)=\sum_{k \in \mathbb{Z}} A c(k-n)(f(x(k))+y(k)), \quad n \in \mathbb{Z} .
\end{gathered}
$$

By (1.44), we have (1.86).

\subsection{Nonlinear operator equation}

DEFINITION 1.18. A sequence $Y:=\{Y(n): n \in \mathbb{Z}\} \subset \mathscr{L}(B)$ is bounded if

$$
\|Y\|_{\infty}:=\sup _{n \in \mathbb{Z}}\|Y(n)\|<+\infty .
$$


Let $A$ be a densely defined operator in $B$ with domain $D(A)$. In this section, we consider one example of the essentially nonlinear difference equation

$$
A X(n)=X(n)(X(n+1)-X(n-1))+Y(n), \quad n \in \mathbb{Z}
$$

for an operator sequence $\{X(n)\}$ and the given bounded sequence of operators $\{Y(n)\}$.

DEFINITION 1.19. A sequence $X:=\{X(n)\}$ of operators is called a solution of (1.98), if

(i) for all $n \in \mathbb{Z}, X(n): B \rightarrow D(A), A X(n) \in \mathscr{L}(B)$;

(ii) equation (1.98) holds.

THEOREM 1.20 (see [19]). Let $A$ be a densely defined operator in $B$ with domain $D(A)$. If there is an operator $W \in \mathscr{L}(B)$ such that

(i) $W: B \rightarrow D(A), A W=I$ on $B$;

(ii) for a sequence $Y$, we have the inequality

$$
8\|W\|^{2}\|Y\|_{\infty} \leq 1 .
$$

Then (1.98) has a bounded solution.

Proof. Let $Y$ be a bounded sequence which satisfies (1.99). We consider a sequence $\left\{X_{m}: m \geq 0\right\}$, for $n \in \mathbb{Z}$, given by

$$
\begin{aligned}
X_{0}(n) & :=W Y(n), \\
X_{m}(n) & :=\sum_{k=0}^{m-1} W X_{m-k-1}(n)\left(X_{k}(n+1)-X_{k}(n-1)\right), \quad m \geq 1 .
\end{aligned}
$$

It can be easily checked that

$$
\begin{aligned}
&\left\|X_{m}\right\|_{\infty} \leq 2\|W\| \sum_{k=0}^{m-1}\left\|X_{m-k-1}\right\|_{\infty}\left\|X_{k}\right\|_{\infty}, \quad m \geq 1 ; \\
&\left\|X_{0}\right\|_{\infty} \leq\|W\| \cdot\|Y\|_{\infty} .
\end{aligned}
$$

By induction and by the following identity for $n \geq 1$

$$
\sum_{k=0}^{n} C_{2 n-2 k}^{n-k} C_{2 k}^{k} \frac{1}{k+1}=\frac{1}{2} C_{2(n+1)}^{n+1},
$$

(see, e.g., [37, Chapter 3, Problems, 11.(a)]) we have

$$
\left\|X_{m}\right\|_{\infty} \leq \frac{1}{m+1} C_{2 m}^{m} 2^{m}\|W\|^{2 m+1}\|Y\|_{\infty}^{m+1}, \quad m \geq 0 .
$$

For $n \geq 2$, the number

$$
\frac{1}{n} C_{2 n-2}^{n-1}
$$

is called the $n$th Catalan number. Then, by (1.99) and (1.103), the series

$$
X(n):=\sum_{k=0}^{\infty} X_{m}(n)
$$


converges in the operator norm for every $n \in \mathbb{Z}$, since

$$
C_{2 m}^{m} \asymp \frac{2^{2 m}}{\sqrt{\pi m}}, \quad m \rightarrow \infty .
$$

By definition, for every $m \geq 0, n \in \mathbb{Z}, N \geq 1$, and $z \in B$,

$$
\begin{gathered}
X_{m}(n) z \in D(A), \quad \sum_{k=0}^{N} X_{m}(n) z \in D(A) \\
A\left(\sum_{m=0}^{N} X_{m}(n) z\right)=Y(n) z+\sum_{m=1}^{N} \sum_{k=0}^{m-1} X_{m-k-1}(n)\left(X_{k}(n+1)-X_{k}(n-1)\right) z .
\end{gathered}
$$

Relation (1.103) implies also the convergence of the series

$$
Y(n) z+\sum_{m=1}^{\infty}\left(\sum_{k=0}^{m-1} X_{m-k-1}(n)\left(X_{k}(n+1)-X_{k}(n-1)\right)\right) z
$$

in norm $B, n \in \mathbb{Z}$. Thus by the closedness of $A$, for every $n \in \mathbb{Z}$,

$$
\begin{aligned}
& X(n) z \in D(A) \\
& A X(n)=Y(n) z+\sum_{m=1}^{\infty}\left(\sum_{k=0}^{m-1} X_{m-k-1}(n)\left(X_{k}(n+1)-X_{k}(n-1)\right)\right) z .
\end{aligned}
$$

Hence

$$
A X(n)=X(n)(X(n+1)-X(n-1))+Y(n), \quad n \in \mathbb{Z} .
$$

REMARKS. (3) The proof of Theorem 1.20 contains the successive approximations for the solution of (1.98).

(4) If $\|Y\|_{\infty}>0$, then the sequence $\{X(n)=\Theta: n \in \mathbb{Z}\}$ is not a solution of (1.98).

\subsection{Stability of solutions under perturbation of operator coefficients}

THEOREM 1.21 (see [11, 12]). Let the operators

$$
\left\{A ; A_{m}(n), n \in \mathbb{Z}, m \geq 1\right\} \subset \mathscr{L}(B)
$$

satisfy the following conditions:

(i) $\sigma(A) \cap S=\varnothing$;

(ii) $\delta_{m}:=\sup \left\{\left\|A_{m}(n)-A\right\| \mid n \in \mathbb{Z}\right\} \rightarrow 0, m \rightarrow \infty$.

Let $\{y(n)\}$ be a bounded sequence in $B$.

Then the equation

$$
x(n+1)=A x(n)+y(n), \quad n \in \mathbb{Z},
$$

and, for every $m$ greater than some $m_{0} \in \mathbb{N}$, the equation

$$
x_{m}(n+1)=A_{m}(n) x_{m}(n)+y(n), \quad n \in \mathbb{Z}
$$

have bounded solutions $\{x(n)\}$ and $\left\{x_{m}(n)\right\}$, respectively, and

$$
\left\|x-x_{m}\right\|_{\infty} \rightarrow 0, \quad m \rightarrow \infty .
$$


Proof. Equation (1.112) has a unique bounded solution by Theorem 1.3. Let $m_{0} \in$ $\mathbb{N}$ such that $L \delta_{m}<1$ for $m \geq m_{0}$, see the proof of Theorem 1.3. To prove the existence of the solution of (1.113) for $m \geq m_{0}$, we construct by Theorem 1.3 the following successive approximations:

$$
\begin{aligned}
x_{m}^{0}(n) & :=\overline{0}, \quad n \in \mathbb{Z} ; \\
x_{m}^{j+1}(n+1) & =A x_{m}^{j+1}(n)+\left(A_{m}(n)-A\right) x_{m}^{j}(n)+y(n), \quad n \in \mathbb{Z}, j \geq 0 .
\end{aligned}
$$

It is easy to verify that

$$
\Delta_{m}^{j} \leq L \delta_{m} \Delta_{m}^{j-1}, \quad j \geq 1
$$

where $\Delta_{m}^{j}:=\left\|x_{m}^{j+1}-x_{m}^{j}\right\|_{\infty}, j \geq 1$. Therefore, for every $n \in \mathbb{Z}$, there is an element $x_{m}(n) \in B$ such that in $B$-norm,

$$
x_{m}^{j}(n) \longrightarrow x_{m}(n), \quad j \longrightarrow \infty ; \quad\left\|x_{m}\right\|_{\infty}<+\infty .
$$

Taking the limit in $j$ in both sides of equality (1.115), we conclude that $x_{m}$ is a solution of (1.113). By (1.112) and (1.113), it follows that

$$
\begin{aligned}
& \left\|x-x_{m}\right\|_{\infty} \leq L \delta_{m}\left\|x_{m}\right\|, \\
& \left\|x-x_{m}\right\|_{\infty} \leq \frac{L \delta_{m}}{1-L \delta_{m}}\|y\|_{\infty}, \quad m \geq 1 .
\end{aligned}
$$

THEOREM 1.22 (see [12]). Let operators from $\mathscr{L}(B)$

$$
A(n), \quad A_{m}(n), \quad n \in \mathbb{Z}, m \geq 1,
$$

satisfy the following conditions for fixed $p \in \mathbb{N}$ :

(i) for all $n \in \mathbb{Z}, A(n+p)=A(n)$;

(ii) $\sigma(A(p-1) A(p-2) \cdots A(1) A(0)) \cap S=\varnothing$;

(iii) $\sup \left(\left\|A_{m}(n)-A(n)\right\| \mid n \in \mathbb{Z}\right) \rightarrow 0, m \rightarrow \infty$.

Let $\{y(n)\}$ be a p-periodic sequence in $B$. Then the equation

$$
x(n+1)=A(n) x(n)+y(n), \quad n \in \mathbb{Z}
$$

and, for every $m$ greater than some $m_{0} \in \mathbb{N}$, the equation

$$
x_{m}(n+1)=A_{m}(n) x_{m}(n)+y(n), \quad n \in \mathbb{Z}
$$

have a unique p-periodic solution $\{x(n)\}$ and a unique bounded solution $\left\{x_{m}(n)\right\}$, respectively; and

$$
\left\|x-x_{m}\right\|_{\infty} \rightarrow 0, \quad m \rightarrow \infty
$$

Proof. By Theorem 1.12, the proof is analogous to that of Theorem 1.21 and is omitted. Some other results about stability of bounded solutions can be found in [10]. 
1.9. Boundary value problem for difference equation. We return to (1.5) and consider the following boundary value problem. Let the integer numbers $\left\{n_{1}, n_{2}\right\} \subset \mathbb{Z}$, $n_{1}<n_{2}$ be given. The boundary value problem to (1.5) is the following. Let $a_{1}, a_{2}$, and $\left\{y\left(n_{1}\right), y\left(n_{1}+1\right), \ldots, y\left(n_{2}-1\right)\right\}$ be arbitrary elements from $B$. It is possible to find elements $\left\{x\left(n_{1}\right), x\left(n_{1}+1\right), \ldots, x\left(n_{2}\right)\right\} \subset B$ such that

$$
\begin{aligned}
x(n+1) & =A x(n)+y(n), \quad n_{1} \leq n \leq n_{2}-1 ; \\
x\left(n_{1}\right) & =a_{1}, \quad x\left(n_{2}\right)=a_{2} .
\end{aligned}
$$

However, it is easy to see that this problem does not have a solution in general. We consider the correct boundary value problem to (1.5) with an operator $A$ satisfying condition (1.6). Let $B_{-}=P_{-} B, B_{+}=P_{+} B$, both $B_{-}$and $B_{+}$are invariant under $A$.

THEOREM 1.23. Let $A$ be an operator satisfying condition (1.6). For arbitrary $a_{1} \in$ $B_{-}, a_{2} \in B_{+}$, and $\left\{y\left(n_{1}\right), y\left(n_{1}+1\right), \ldots, y\left(n_{2}-1\right)\right\} \subset B$, the following problem:

$$
\begin{aligned}
& x(n+1)=A x(n)+y(n), \quad n_{1} \leq n \leq n_{2}-1 ; \\
& P_{-} x\left(n_{1}\right)=a_{1}, \quad P_{+} x\left(n_{2}\right)=a_{2}
\end{aligned}
$$

has a unique solution $\left\{x\left(n_{1}\right), x\left(n_{1}+1\right), \ldots, x\left(n_{2}\right)\right\}$.

Proof. Ignoring a trivial case, we suppose that $\sigma_{-} \neq \varnothing$ and $\sigma_{+} \neq \varnothing$. Let $A_{-}:=A P_{-}$, $A_{+}:=A P_{+}$, and $x_{-}:=P_{-} x, x_{+}:=P_{+} x$ for $x \in B$. If $\left\{x\left(n_{1}\right), x\left(n_{1}+1\right), \ldots, x\left(n_{2}\right)\right\}$ is a solution of (1.124) in $B$, then $\left\{x_{-}\left(n_{1}\right), x_{-}\left(n_{1}+1\right), \ldots, x_{-}\left(n_{2}\right)\right\}$ is a solution of the problem

$$
\begin{aligned}
x_{-}(n+1) & =A_{-} x_{-}(n)+y_{-}(n), \quad n_{1} \leq n \leq n_{2}-1 ; \\
x_{-}\left(n_{1}\right) & =a_{1},
\end{aligned}
$$

in $B_{-}$; and $\left\{x_{+}\left(n_{1}\right), x_{+}\left(n_{1}+1\right), \ldots, x_{+}\left(n_{2}\right)\right\}$ is a solution of the problem

$$
\begin{aligned}
x_{+}(n+1) & =A_{+} x_{+}(n)+y_{+}(n), \quad n_{1} \leq n \leq n_{2}-1 ; \\
x_{+}\left(n_{2}\right) & =a_{2},
\end{aligned}
$$

in $B_{+}$. Rewrite (1.126) as

$$
\begin{aligned}
x_{+}(n) & =A_{+}^{-1} x_{+}(n+1)-A_{+}^{-1} y_{+}(n), \quad n_{1} \leq n \leq n_{2}-1 ; \\
x_{+}\left(n_{2}\right) & =a_{2} .
\end{aligned}
$$

Problem (1.125) is an initial-value problem and problem (1.127) is a reverse initialvalue problem. It is trivial that

$$
\begin{aligned}
& x_{-}(n)=\sum_{j=0}^{n-n_{1}-1} A_{-}^{j} y_{-}(n-j-1)+A_{-}^{n-n_{1}} a_{1}, \quad n>n_{1}, \\
& x_{+}(n)=-\sum_{j=n-n_{2}}^{-1} A_{+}^{j} y_{+}(n-j-1), \quad n<n_{2} .
\end{aligned}
$$

It is easy to see that $\left\{x(n):=x_{-}(n)+x_{+}(n), n_{1} \leq n_{2}\right\}$ is a unique solution to (1.124). 
Write $x\left(n ; n_{1}, n_{2}, a_{1}, a_{2}\right):=x(n)$ for a solution of problem (1.124) with $n_{1} \leq n \leq n_{2}$.

THEOREM 1.24. Let $A$ be an operator satisfying condition (1.6) and let $\{y(n)\}$ be a bounded sequence in $B$. For any $n \in \mathbb{Z}$ and $c>0$,

$$
\lim _{n_{1} \rightarrow-\infty, n_{2} \rightarrow+\infty} \sup _{\left\|a_{1}\right\| \leq c,\left\|a_{2}\right\| \leq c}\left\|x\left(n ; n_{1}, n_{2}, a_{1}, a_{2}\right)-z(n)\right\|=0,
$$

where $z$ is a unique bounded solution to (1.5).

PROoF. This follows from the proofs of Theorems 1.3 and 1.23.

2. Periodic random sequences in Banach spaces. Strictly periodic in distribution random sequences, second-order periodic random sequences and their connection between themselves and with stationary sequences are the topics of this section. We discuss the basic properties of the periodic in distribution random sequences in a Banach space. The second-order periodic and weakly second-order periodic processes in a Hilbert space are treated. The results of Sections 1 and 2 are the basis to study the stochastic difference equations with periodic disturbances or periodic structure. The definition of the second-order periodic $\mathbb{C}$-valued process has been introduced by Gladyshev [18], where spectral properties of second-order periodic process have also been considered. For Hilbert-valued process, it is natural to consider two definitions for the second-order periodicity which are identical in finite-dimensional space. For more details see [9]. Periodicity often arises in economic and geophysical time series, see the article by Troutman [40], where sufficient conditions for existence of the real periodic solution to difference equations are given. In Jiri [24, 25], the methods for the statistical analysis of the periodic autoregressive processes in finite-dimensional space are given. Periodic in distribution and the second-order periodic random processes have been considered by Morozan [33]. The statistical problems including the estimation of correlation function for the second-order periodic processes have been investigated by Pagano [36]. Almost periodic in distribution processes with discrete time have also been considered [21, 33]. Some methods of analysis of stationary random sequences in a Banach space can be found in [3, 31, 34].

2.1. Periodic in distribution random sequences. Let $(B,\|\cdot\|)$ be a complex separable Banach space with the zero element $\overline{0}$ and let $B^{*}$ be its dual space. Let $\mathscr{B}(B)$ be the Borel $\sigma$-algebra on $B$. We refer to [27] for the basic $B$-valued random variables or random elements theory. All random elements which arise in the next sections are considered on a complete probability space $(\Omega, \mathscr{F}, \mathbf{P})$. Further, all equalities, inequalities, and so on, with the random elements or random variables mean those almost surely.

DEFINITION 2.1. The mapping

$$
x: \mathbb{Z} \times \Omega \rightarrow B
$$

is called a $B$-valued random process with discrete time or random sequence in $B$ if, for each $n \in \mathbb{Z}$, the mapping

$$
x(n):=x(n, \cdot): \Omega \rightarrow B
$$


is a $B$-valued random element. For each $\omega \in \Omega$, the sequence $\{x(n, \omega): n \in \mathbb{Z}\}$ is called trajectory of the random process:

$$
\{x(n): n \in \mathbb{Z}\} \quad \text { or } \quad\{x(n, \omega): n \in \mathbb{Z}\} .
$$

Let

$$
B^{\mathbb{Z}}:=\{\{u(n): n \in \mathbb{Z}\} \mid \forall n \in \mathbb{Z}: u(n) \in B\}
$$

be the set of all sequences with elements from $B$; and, for each $j \in \mathbb{Z}$, let

$$
\pi_{j}(\{u(n): n \in \mathbb{Z}\}):=u(j) .
$$

The $\sigma$-algebra in the space $B^{\mathbb{Z}}$ is defined as

$$
\mathscr{B}^{\mathbb{Z}}:=\sigma a\left(\bigcup_{j \in \mathbb{Z}} \pi_{j}^{-1}(\mathscr{B}(B))\right) .
$$

For every random process $\{x(n): n \in \mathbb{Z}\}$ there is a correspondent probability measure $\mu_{x}$ on $\mathscr{B}^{\mathbb{Z}}$, which is defined by the equality

$$
\mu_{x}(A):=\mathbf{P}[\omega \mid\{x(n, \omega): n \in \mathbb{Z}\} \in A], \quad A \in \mathscr{B}^{\mathbb{Z}} .
$$

By the well-known Kolmogorov theorem, see, for instance, [17], the measure $\mu_{x}$ is uniquely restored by collection of the finite-dimensional distributions

$$
\left\{\mu_{x}^{I}: I \subset \mathbb{Z}, I \text { is finite set }\right\},
$$

where for every given $I$, the measure $\mu_{x}^{I}$ is a unique extension on

$$
\sigma a\left(\bigcup_{j \in I} \pi_{j}^{-1}(\mathscr{B}(B))\right)
$$

of the function

$$
\mu_{x}^{I}\left(\prod_{j \in I} A_{j}\right):=\mathbf{P}\left[\bigcap_{j \in I}\left\{\omega \mid x(j, \omega) \in A_{j}\right\}\right], \quad A_{j} \in \mathscr{B}(B), j \in I .
$$

Define, for every $j \in \mathbb{Z}$, the map $\theta(j): B^{\mathbb{Z}} \rightarrow B^{\mathbb{Z}}$ as

$$
\theta(j)(\{x(n): n \in \mathbb{Z}\}):=\{x(n+j): n \in \mathbb{Z}\} .
$$

The map $\theta(j)$ is one-to-one and the maps $\theta(j)$ and $\theta(j)^{-1}$ are measurable. For a random process $\{x(n): n \in \mathbb{Z}\}$, let

$$
\mu_{\theta(j) x}
$$

be the measure which corresponds to the process

$$
\theta(j)\{x(n): n \in \mathbb{Z}\}:=\{x(n+j): n \in \mathbb{Z}\} .
$$


We have

$$
\mu_{\theta(j) x}=\mu_{x} \theta(j)^{-1} .
$$

Let a number $p \in \mathbb{N}$ be fixed.

DEFINITION 2.2. A $B$-valued random process $\{x(n): n \in \mathbb{Z}\}$ is called periodic of the period $p$ in distribution or $p$-periodic in distribution, if, for every

$$
n \in \mathbb{N}, \quad\left\{m_{1}, m_{2}, \ldots, m_{n}\right\} \subset \mathbb{Z}, \quad\left\{A_{1}, A_{2}, \ldots, A_{n}\right\} \subset \mathscr{B}(B),
$$

the function

$$
\mathbb{Z} \ni n \longmapsto \mathbf{P}\left[\bigcap_{j=1}^{n}\left\{\omega \mid x\left(m_{j}+n, \omega\right) \in A_{j}\right\}\right]
$$

is periodic on $\mathbb{Z}$ of period $p$.

REMARKS. (1) A periodic of period $p=1$ in distribution random processes is stationary.

(2) A stationary random process is periodic of period $p$ in distribution for every $p \in \mathbb{N}$.

DEFINITION 2.3. The $B$-valued random processes

$$
\left\{x_{j}(n): n \in \mathbb{Z}\right\}, \quad 1 \leq j \leq m,
$$

are called periodically with period $p$ connected or periodically connected, if the $B^{m}$ valued random process

$$
\left\{\left(x_{1}(n), x_{2}(n), \ldots, x_{m}(n)\right): n \in \mathbb{Z}\right\}
$$

is periodic of period $p$ in distribution.

We refer to [9, Chapter 2] for basic properties of periodic in distribution processes and list only several that we need later.

LEMmA 2.4. A process $\{x(n): n \in \mathbb{Z}\}$ is periodic in distribution of period $p$ if and only if

$$
\mu_{x} \theta(p)^{-1}=\mu_{x}
$$

Proof. By the Kolmogorov theorem, it is sufficient to prove equality (2.19) only for finite-dimensional distributions (2.8); and, therefore, by uniqueness of an extension of a measure, it suffices to prove (2.19) for functions (2.10). See Definition 2.2.

LEMMA 2.5. A $B$-valued process $\{x(n): n \in \mathbb{Z}\}$ is periodic of period $p$ in distribution if and only if the $B^{p}$-valued process

$$
\{\vec{x}(n):=(x(n p), x(n p+1), \ldots, x(n p+p-1)): n \in \mathbb{Z}\}
$$

is stationary.

Proof. We first show that if $\{x(n): n \in \mathbb{Z}\}$ is $p$-periodic in distribution, then process (2.20) is stationary. Let

$$
n \in \mathbb{N}, \quad\left\{m_{1}, \ldots, m_{n}\right\} \subset \mathbb{Z}, \quad A_{1}, \ldots, A_{n},
$$


where

$$
A_{j}=A_{j 0} \times \cdots \times A_{j(p-1)}, \quad A_{j k} \in \mathscr{B}(B), 1 \leq j \leq n, 0 \leq k \leq p-1
$$

is given. By uniqueness of an extension of measure from semi-algebra to the $\sigma$-algebra generated by semi-algebra, (see, e.g., [22] and Definition 2.2) we have

$$
\begin{aligned}
\mathbf{P}\left[\bigcap_{j=1}^{n}\left\{\omega \mid \vec{x}\left(m_{j}+1\right) \in A_{j}\right\}\right] & =\mathbf{P}\left[\bigcap_{j=1}^{n} \bigcap_{k=0}^{p-1}\left\{\omega \mid x\left(\left(m_{j}+1\right) p+k\right) \in A_{j k}\right\}\right] \\
& =\mathbf{P}\left[\bigcap_{j=1}^{n} \bigcap_{k=0}^{p-1}\left\{\omega \mid x\left(m_{j} p+k\right) \in A_{j k}\right\}\right] \\
& =\mathbf{P}\left[\bigcap_{j=1}^{n}\left\{\omega \mid \vec{x}\left(m_{j}\right) \in A_{j}\right\}\right] .
\end{aligned}
$$

Now, assume that (2.20) is stationary. Given any

$$
n \in \mathbb{N}, \quad\left\{m_{1}, \ldots, m_{n}\right\} \subset \mathbb{Z}, \quad\left\{A_{1}, \ldots, A_{n}\right\} \subset \mathscr{B}(B),
$$

put $m_{j}=\left(p_{j}+1\right) p+k_{j}$, where $\left\{p_{1}, \ldots, p_{n}\right\} \subset \mathbb{Z}$ and $0 \leq k_{j} \leq p-1,1 \leq j \leq n$. For the sets

$$
C_{j l}:= \begin{cases}A_{j}, & l=k_{j} ; \\ B, & l \neq k_{j} ;\end{cases}
$$

where $1 \leq j \leq n, 0 \leq l \leq p-1$, and

$$
C_{j}:=C_{j 0} \times \cdots \times C_{j(p-1)}, \quad 0 \leq j \leq n,
$$

from Definition 2.2 for (2.20), we have

$$
\begin{aligned}
\mathbf{P}\left[\bigcap_{j=1}^{n}\left\{\omega \mid x\left(m_{j}+p\right) \in A_{j}\right\}\right] & =\mathbf{P}\left[\bigcap_{j=1}\left\{\omega \mid x\left(\left(p_{j}+1\right) p+k_{j}\right) \in A_{j}\right\}\right] \\
& =\mathbf{P}\left[\bigcap_{j=1}^{n}\left\{\omega \mid \vec{x}\left(p_{j}+1\right) \in C_{j}\right\}\right] \\
& =\mathbf{P}\left[\bigcap_{j=1}\left\{\omega \mid \vec{x}\left(p_{j}\right) \in C_{j}\right\}\right] \\
& =\mathbf{P}\left[\bigcap_{j=1}^{n}\left\{\omega \mid x\left(m_{j}\right) \in A_{J}\right\}\right] .
\end{aligned}
$$

THEOREM 2.6. Let $\{x(n): n \in \mathbb{Z}\}$ be a p-periodic in distribution random process in $B, B_{1}$ a complex separable Banach space, and $m \in \mathbb{N}$. Suppose that a map

$$
g: \mathbb{Z} \times B^{m} \longrightarrow B_{1}
$$


satisfies the conditions

$$
\forall n \in \mathbb{Z} \quad \forall \vec{x} \in B^{m}: g(n+p, \vec{x})=g(n, \vec{x}) ; \quad g(n, \cdot) \in C\left(B^{m}, B_{1}\right) .
$$

Then, for every $n_{1}, \ldots, n_{m}$ from $\mathbb{Z}$, the $B_{1}$-valued random process

$$
\left\{g\left(n, x\left(n+n_{1}\right), x\left(n+n_{2}\right), \ldots, x\left(n+n_{m}\right)\right): n \in \mathbb{Z}\right\}
$$

is p-periodic in distribution.

Proof. Let

$$
k \in \mathbb{N}, \quad\left\{t_{1}, \ldots, t_{k}\right\} \subset \mathbb{Z}, \quad\left\{A_{1}, \ldots, A_{k}\right\} \subset \mathscr{B}\left(B_{1}\right)
$$

be given. We have, clearly,

$$
C_{j}:=\left\{\vec{x} \in B^{m} \mid g\left(t_{j}+p, \vec{x}\right) \in A_{j}\right\}=\left\{\vec{x} \in B^{m} \mid g\left(t_{j}, \vec{x}\right) \in A_{j}\right\}, \quad 1 \leq j \leq k .
$$

Define for $j, 1 \leq j \leq k$,

$$
\vec{x}\left(t_{j}\right):=\left(x\left(t_{j}+n_{1}\right), x\left(t_{j}+n_{2}\right), \ldots, x\left(t_{j}+n_{m}\right)\right) .
$$

To prove the theorem, we use Definition 2.2 to show that

$$
\mathbf{P}\left[\bigcap_{j=1}^{k}\left\{\omega \mid \vec{x}\left(t_{j}+p\right) \in C_{j}\right\}\right]=\mathbf{P}\left[\bigcap_{j=1}^{k}\left\{\omega \mid \vec{x}\left(t_{j}\right) \in C_{j}\right\}\right] .
$$

By periodicity of the process $\{x(n): n \in \mathbb{Z}\}$, equality (2.34) holds for the sets

$$
C_{j}=C_{j 1} \times \cdots \times C_{j m}, \quad C_{j l} \in \mathscr{B}(B), 1 \leq j \leq k, 1 \leq l \leq m .
$$

Therefore, (2.34) holds by the uniqueness of extension of measure.

COROLLARY 2.7. Let $\{x(n): n \in \mathbb{Z}\}$ be a p-periodic in distribution random process in $B$.

(i) The process

$$
\{\|x(n)\|: n \in \mathbb{Z}\}
$$

is $p$-periodic in distribution in $\mathbb{R}$.

(ii) For any $g \in B^{*}$, the process

$$
\{\langle g, x(n)\rangle: n \in \mathbb{Z}\}
$$

is $p$-periodic in distribution in $\mathbb{C}$.

(iii) If a function $a: \mathbb{Z} \rightarrow B$ is $p$-periodic, then the process

$$
\{a(n)+x(n): n \in \mathbb{Z}\}
$$

is p-periodic in distribution in $B$. 
(iv) If a function $A: \mathbb{Z} \rightarrow \mathscr{L}\left(B, B_{1}\right)$ is $p$-periodic, then the process

$$
\{A(n) x(n): n \in \mathbb{Z}\}
$$

is $p$-periodic in distribution in $B_{1}$.

(v) If the processes $\left\{x_{j}(n): n \in \mathbb{Z}\right\}, j=1,2$ are periodically connected with period $p$, then the process

$$
\left\{x_{1}(n)+x_{2}(n): n \in \mathbb{Z}\right\}
$$

is $p$-periodic in distribution in $B$.

TheOREM 2.8. Let $m \in \mathbb{N}$ and let $\{x(n): n \in \mathbb{Z}\}$ be $p$-periodic in distribution in $B$. Suppose that $F \in C\left(B^{m}, \mathbb{C}\right)$ and numbers $\left\{n_{1}, \ldots, n_{m}\right\} \subset \mathbb{Z}$ such that

$$
\mathrm{E}\left|F\left(x\left(n_{1}\right), \ldots, x\left(n_{m}\right)\right)\right|<+\infty .
$$

Then

$$
\forall j \in \mathbb{Z}: \mathbf{E} F\left(x\left(n_{1}+j p\right), \ldots, x\left(n_{m}+j p\right)\right)=\mathbf{E} F\left(x\left(n_{1}\right), \ldots, x\left(n_{m}\right)\right) .
$$

PRoof. We establish the result for $j=1$. For $\left\{n_{1}, \ldots, n_{m}\right\} \subset \mathbb{Z}, m \in \mathbb{N}$, let $\mu\left(n_{1}, \ldots\right.$, $\left.n_{m} ; \cdot\right)$ be the distribution on $\mathscr{B}\left(B^{m}\right)$ of the random element $\left(x\left(n_{1}\right), \ldots, x\left(n_{m}\right)\right)$. Note that

$$
\mu\left(n_{1}, \ldots, n_{m} ; A_{1} \times \cdots \times A_{m}\right)=\mathbf{P}\left[\bigcap_{k=1}^{m}\left\{\omega: x\left(n_{k}\right) \in A_{k}\right\}\right],
$$

for every measurable parallelepiped:

$$
A_{1} \times A_{2} \times \cdots \times A_{m}, \quad\left\{A_{1}, \ldots, A_{m}\right\} \subset \mathscr{B}(B) .
$$

Then, according to Definition 2.2, we have

$$
\mu\left(n_{1}, \ldots, n_{m} ; A_{1} \times \cdots \times A_{m}\right)=\mu\left(n_{1}+p, \ldots, n_{m}+p ; A_{1} \times \cdots \times A_{m}\right) .
$$

Therefore, the measures $\mu\left(n_{1}, \ldots, n_{m} ; \cdot\right)$ and $\mu\left(n_{1}+p, \ldots, n_{m}+p\right)$ coincide, since the $\sigma$-algebra $\mathscr{B}\left(B^{m}\right)$ is generated by measurable parallelepipeds. Then

$$
\begin{aligned}
\mathbf{E} F\left(x\left(n_{1}\right), \ldots, x\left(n_{m}\right)\right) & =\int_{B^{m}} F\left(u_{1}, \ldots, u_{m}\right) d \mu\left(n_{1}, \ldots, n_{m} ; \cdot\right) \\
& =\int_{B^{m}} F\left(u_{1}, \ldots, u_{m}\right) d \mu\left(n_{1}+p, \ldots, n_{m}+p ; \cdot\right) \\
& =\mathbf{E} F\left(x\left(n_{1}+p\right), \ldots, x\left(n_{m}+p\right)\right) .
\end{aligned}
$$

COROLLARY 2.9. Let $\{x(n): n \in \mathbb{Z}\}$ be $p$-periodic in distribution in $B$ and

$$
\mathbf{E}|| x(k) \|^{\alpha}<+\infty, \quad 1 \leq k \leq p,
$$

where $\alpha>0$.

Then the function

$$
\mathbb{Z} \ni n \longmapsto \mathbf{E}\|x(n)\|^{\alpha}
$$

is $p$-periodic on $\mathbb{Z}$. 
THEOREM 2.10. The following assertions are equivalent:

(i) a process $\{x(n): n \in \mathbb{Z}\}$ is $p$-periodic in distribution in $B$;

(ii) for all $m \in \mathbb{N}$, for all $\left\{n_{1}, \ldots, n_{m}\right\} \subset \mathbb{Z}$, for all $F \in C\left(B^{m}\right)$, $\sup _{B^{m}}|F|<+\infty$, the function

$$
\mathbb{Z} \ni n \longmapsto \mathbf{E} F\left(x\left(n_{1}+n\right), \ldots, x\left(n_{m}+n\right)\right)
$$

is $p$-periodic.

Proof. (i) implies (ii). This follows directly from Theorem 2.8.

(ii) implies (i). It is enough to show that the measures $\mu\left(n_{1}, \ldots, n_{m} ; \cdot\right)$ and $\mu\left(n_{1}+\right.$ $\left.p, \ldots, n_{m}+p\right)$ are equal for every $m \in \mathbb{N}$ and $\left\{n_{1}, \ldots, n_{m}\right\} \subset \mathbb{Z}$. Therefore, we may equivalently prove the equality of the corresponding characteristic functions, see [2]. However, by Theorem 2.8, it follows that for every $g \in\left(B^{m}\right)^{*}$,

$$
\begin{aligned}
\hat{\mu}\left(n_{1}, \ldots, n_{m}\right)(g): & =\operatorname{Eexp}\left(i \operatorname{Re}\left\langle g,\left(x\left(n_{1}\right), \ldots, x\left(n_{m}\right)\right)\right\rangle\right) \\
& =\exp \left(i \operatorname{Re}\left\langle g,\left(x\left(n_{1}+p\right), \ldots, x\left(n_{m}+p\right)\right)\right\rangle\right) \\
& =: \hat{\mu}\left(n_{1}+p, \ldots, n_{m}+p\right)(g) .
\end{aligned}
$$

COROLlaRY 2.11. A random process $\{x(n): n \in \mathbb{Z}\}$ in $B$ is $p$-periodic in distribution, if and only if the functions

$$
\begin{aligned}
& \mathbb{Z} \ni n \longmapsto \mathrm{E} \cos \left(\sum_{j=1}^{m} \operatorname{Re}\left\langle g_{j}, x\left(n_{j}+n\right)\right\rangle\right), \\
& \mathbb{Z} \ni n \longmapsto \mathrm{E} \sin \left(\sum_{j=1}^{m} \operatorname{Re}\left\langle g_{j}, x\left(n_{j}+n\right)\right\rangle\right)
\end{aligned}
$$

are $p$-periodic on $\mathbb{Z}$ for every

$$
m \in \mathbb{N}, \quad\left\{n_{1}, \ldots, n_{m}\right\} \subset \mathbb{Z}, \quad\left\{g_{1}, \ldots, g_{m}\right\} \subset B^{*} .
$$

THEOREM 2.12. Let $\{x(n): n \in \mathbb{Z}\}$ be a p-periodic in distribution process in $B$ and let a function $f: B^{\mathbb{Z}} \rightarrow B_{1}$ be $\mathscr{B}^{\mathbb{Z}}-\mathscr{B}_{B}\left(B_{1}\right)$ be measurable.

Then the process

$$
\{f(\{x(n+k): k \in \mathbb{Z}\}): n \in \mathbb{Z}\}
$$

is $p$-periodic in distribution in $B_{1}$.

Proof. Given

$$
m \in \mathbb{N}, \quad\left\{n_{1}, \ldots, n_{m}\right\} \subset \mathbb{Z}, \quad\left\{A_{1}, \ldots, A_{m}\right\} \subset \mathscr{B}\left(B_{1}\right),
$$

define

$$
C_{k}:=f^{-1}\left(A_{k}\right), \quad C_{k} \in \mathscr{B}^{\mathbb{Z}}, 1 \leq k \leq m .
$$


It is obvious that

$$
\begin{aligned}
\mathbf{P}\left[\bigcap_{k=1}^{m}\{\omega \mid f\right. & \left.\left.\left.\left\{x\left(n_{k}+j\right): j \in \mathbb{Z}\right\}\right) \in A_{k}\right\}\right] \\
& =\mathbf{P}\left[\bigcap_{k=1}^{m}\left\{\omega \mid\left\{x\left(n_{k}+j\right): j \in \mathbb{Z}\right\} \in C_{k}\right\}\right] \\
& =\mathbf{P}\left[\bigcap_{k=1}^{m}\left\{\omega \mid \theta\left(n_{k}\right)\{x(j): j \in \mathbb{Z}\} \in C_{k}\right\}\right] \\
& =\mathbf{P}\left[\bigcap_{k=1}^{m}\left\{\omega \mid\{x(j): j \in \mathbb{Z}\} \in \theta\left(-n_{k}\right) C_{k}\right\}\right] \\
& =\mathbf{P}\left[\left\{\omega \mid\{x(j): j \in \mathbb{Z}\} \in \bigcap_{k=1}^{m} \theta\left(-n_{k}\right) C_{k}\right\}\right] \\
& =: \mu_{x}\left(\bigcap_{k=1}^{m} \theta\left(-n_{k}\right) C_{k}\right) .
\end{aligned}
$$

By Lemma 2.4, we have

$$
\mu_{x}\left(\bigcap_{k=1}^{m} \theta\left(-n_{k}\right) C_{k}\right)=\mu_{x}\left(\bigcap_{k=1}^{m} \theta\left(-n_{k}-p\right) C_{k}\right) ;
$$

and, it follows from (2.56), that

$$
\begin{aligned}
\mathbf{P}\left[\bigcap_{k=1}^{m}\left\{\omega \mid f\left(\left\{x\left(n_{k}+j\right): j \in \mathbb{Z}\right\}\right) \in A_{k}\right\}\right] \\
=\mathbf{P}\left[\bigcap_{k=1}^{m}\left\{\omega \mid f\left(\left\{x\left(n_{k}+p+j\right): j \in \mathbb{Z}\right\}\right) \in A_{k}\right\}\right] .
\end{aligned}
$$

EXAMPLE 2.13. Let $\{x(n): n \in \mathbb{Z}\}$ be a $p$-periodic in distribution process such that

$$
\mathbf{E}|| x(k) \|<+\infty, \quad 1 \leq k \leq p .
$$

Assume that

$$
\{A(k): k \in \mathbb{Z}\} \subset \mathscr{L}(B), \quad \sum_{k \in \mathbb{Z}}\|A(k)\|<+\infty .
$$

Then, for every $n \in \mathbb{Z}$, the series

$$
y(n):=\sum_{k \in \mathbb{Z}} A(k) x(n-k)
$$

converges almost surely in $B$-norm and $\{y(n): n \in \mathbb{Z}\}$ is $p$-periodic in distribution.

THEOREM 2.14. Let $\left\{x_{t}(n): n \in \mathbb{Z}\right\}, t \in \mathbb{N}$, be a sequence of $p$-periodic in distribution processes and let $\{x(n): n \in \mathbb{Z}\}$ be a process such that, for every $n \in \mathbb{Z}$,

$$
\forall \varepsilon>0: \mathbf{P}\left[\left\|x_{t}(n)-x(n)\right\| \geq \varepsilon\right] \longrightarrow 0, \quad t \rightarrow \infty .
$$


Then $\{x(n): n \in \mathbb{Z}\}$ is $p$-periodic in distribution in $B$.

ProOF. Let

$$
\mu_{t}\left(n_{1}, \ldots, n_{m} ; \cdot\right), \quad \mu\left(n_{1}, \ldots, n_{m} ; \cdot\right)
$$

be the distributions of the random elements

$$
\left(x_{t}\left(n_{1}\right), \ldots, x_{t}\left(n_{m}\right)\right), \quad\left(x\left(n_{1}\right), \ldots, x\left(n_{m}\right)\right),
$$

respectively, for $m \in \mathbb{N},\left\{n_{1}, \ldots, n_{m}\right\} \subset \mathbb{Z}$. Then

$$
\mu_{t}\left(n_{1}, \ldots, n_{m} ; \cdot\right) \rightarrow \mu\left(n_{1}, \ldots, n_{m} ; \cdot\right), \quad t \rightarrow \infty
$$

in distribution. Hence, for every

$$
F \in C\left(B^{m}, \mathbb{C}\right), \quad \sup _{B^{m}}|F|<+\infty,
$$

we have

$$
\mathbf{E} F\left(x_{t}\left(n_{1}\right), \ldots, x_{t}\left(n_{m}\right)\right) \rightarrow \mathbf{E} F\left(x\left(n_{1}\right), \ldots, x\left(n_{m}\right)\right), \quad t \rightarrow \infty .
$$

By (2.67) and by Theorem 2.8,

$$
\mathbf{E} F\left(x\left(n_{1}+p\right), \ldots, x\left(n_{m}+p\right)\right)=\mathbf{E} F\left(x\left(n_{1}\right), \ldots, x\left(n_{m}\right)\right) .
$$

Therefore, by Theorem $2.10\{x(n): n \in \mathbb{Z}\}$ is $p$-periodic in distribution.

EXAMPLE 2.15. Let $\{x(n): n \in \mathbb{Z}\}$ be a $p$-periodic in distribution process in $B$ such that

$$
\mathbf{E}\|x(k)\|<+\infty, \quad 1 \leq k \leq p .
$$

Let $\left\{\xi_{k}: k \in \mathbb{Z}\right\}$ be a stationary process in $\mathbb{C}$ such that $\mathbf{E}\left|\xi_{0}\right|<+\infty$. If the processes $\{x(n)\}$ and $\left\{\xi_{k}\right\}$ are independent, then the processes

$$
y(n):=\sum_{k=-\infty}^{n} \frac{\xi_{n-k}}{1+(n-k)^{2}} x(k), \quad z(n):=\sum_{k \in \mathbb{Z}} \frac{\xi_{k}}{1+k^{2}} x(n-k) ; \quad n \in \mathbb{Z},
$$

are $p$-periodic in distribution.

2.2. Second-order periodic random process in Hilbert spaces. In this section, we develop the concept of a second-order periodic random process and give some of its basic properties. Let $(\Omega, \mathscr{F}, \mathbf{P})$ be a complete probability space, and let $(H,(\cdot, \cdot))$ be a complex separable Hilbert space with inner product $(\cdot, \cdot)$ and corresponding norm $\|\cdot\|$.

Let $x(k)$ and $x(n)$ be $H$-valued random variables such that

$$
\mathbf{E}\|x(k)\|^{2}<+\infty, \quad \mathbf{E}\|x(n)\|^{2}<+\infty .
$$


Then the Bochner integrals

$$
\mathbf{E} x(k)=\int_{H} x(k) d \mathbf{P}, \quad \mathbf{E} x(n)=\int_{H} x(n) d \mathbf{P}
$$

are well defined. Define

$$
r(k, n):=\mathbf{E}(x(k)-\mathbf{E} x(k), x(n)-\mathbf{E} x(n))
$$

The function

$$
b_{k, n}(u, v):=\mathbf{E}((x(k)-\mathbf{E} x(k), u) \overline{(x(n)-\mathbf{E} x(n), v)}), \quad\{u, v\} \subset H
$$

is a continuous bilinear mapping. By the Riesz theorem, there is a unique linear bounded operator $S_{k, n}$ such that

$$
b_{k, n}(u, v)=\left(u, S_{k, n} v\right), \quad\{u, v\} \subset H .
$$

The operator $S_{k, n}$ is called joint covariance of $x(k)$ and $x(n)$. It is known (see, e.g., $[28,39])$ that

(i) $S_{k, n}^{*}=S_{n, k}$;

(ii) the operator $S_{n, n}$ is selfadjoint nonnegative and nuclear;

(iii) the following relations hold:

$$
\begin{gathered}
\operatorname{tr} S_{n, n}=\mathbf{E}\|x(n)-\mathbf{E} x(n)\|^{2}, \quad \operatorname{tr}\left(S_{k, n}^{*} S_{k, n}\right)^{1 / 2}<+\infty, \\
\left|\left(u, S_{k, n} v\right)\right|^{2} \leq\left(S_{k, k} u, u\right)\left(S_{n, n} v, v\right) ; \quad\{u, v\} \subset H ;
\end{gathered}
$$

(iv) for every orthonormal basis $\left\{e_{j}: j \geq 1\right\}$, the equality

$$
r(k, n)=\sum_{j=1}^{\infty}\left(e_{j}, S_{k, n} e_{j}\right)
$$

holds.

DEFINITION 2.16. Let $\{x(n): n \in \mathbb{Z}\}$ be an $H$-valued random process such that

$$
\forall n \in \mathbb{Z}: \mathbf{E}\|x(n)\|^{2}<+\infty .
$$

The process $\{x(n): n \in \mathbb{Z}\}$ is called weakly second-order (WSO) $p$-periodic, if

(i) for all $n \in \mathbb{Z}, \mathrm{E} x(n+p)=\mathrm{E} x(n)$;

(ii) for all $\{k, n\} \subset \mathbb{Z}, r(k+p, n+p)=r(k, n)$.

A WSO 1-periodic process is called WSO stationary.

LEMMA 2.17. If $\{x(n): n \in \mathbb{Z}\}$ is WSO p-periodic in $H$, then the $H^{p}$-valued process

$$
\vec{x}(n):=(x(n p), x(n p+1), \ldots, x(n p+p-1)), \quad n \in \mathbb{Z}
$$

is WSO stationary. 
Proof. Let $\{x(n): n \in \mathbb{Z}\}$ be a WSO $p$-periodic process in $H$. It can be easily checked that $\mathrm{E} \vec{x}(n)=\mathrm{E} \vec{x}(0), n \in \mathbb{Z}$ and

$$
\begin{aligned}
\mathbf{E}(\vec{x}(n)-\mathbf{E} \vec{x}(n), \vec{x}(m)-\mathbf{E} \vec{x}(m)) & =\sum_{j=0}^{p-1} r(n p+j, m p+j) \\
& =\sum_{j=0}^{p-1} r((n-m) p+j, j) .
\end{aligned}
$$

REMARK 2.18. If $p \geq 2$ then the converse to Lemma 2.17 is not true. Indeed, let $H=\mathbb{R}^{2}$ and $\left\{\xi_{n}: n \in \mathbb{Z}\right\}$ be a sequence of independent identically distributed real random variables with $\mathbf{E} \xi_{0}=0, \mathbf{E} \xi_{0}^{2}=1$. Define

$$
\vec{x}(n):=(x(2 n), x(2 n+1)), \quad n \in \mathbb{Z},
$$

where

$$
x(2 n):=\xi_{n} ; \quad x(2 n+1):=\xi_{n}, \quad n \in \mathbb{Z} \backslash\{1,2\}, \quad x(3):=\xi_{2}, \quad x(5):=\xi_{1} .
$$

Then the process $\{\vec{x}(n): n \in \mathbb{Z}\}$ is WSO stationary since $\mathbf{E} \vec{x}(n)=(0,0)$ for all $n \in \mathbb{Z}$, and

$$
\begin{aligned}
\mathrm{E}(\vec{x}(n), \vec{x}(m)) & =\mathbf{E} x((2 n) x(2 m))+\mathbf{E}(x(2 n+1) x(2 m+1)) \\
& =2 \delta(n-m), \quad\{n, m\} \subset \mathbb{Z} .
\end{aligned}
$$

However, the process $\{x(n): n \in \mathbb{Z}\}$ is not WSO 2-periodic since

$$
\mathbf{E}(x(0) x(1))=1, \quad \mathbf{E} x(4) x(5)=0 .
$$

DEFINITION 2.19. Let $\{x(n): n \in \mathbb{Z}\}$ be a random process in $H$ such that

$$
\mathbf{E}\|x(n)\|^{2}<+\infty, \quad n \in \mathbb{Z} .
$$

The process $\{x(n): n \in \mathbb{Z}\}$ is called second-order (SO) $p$-periodic if

(i) for all $n \in \mathbb{Z}, \mathrm{E} x(n+p)=\mathrm{E} x(n)$;

(ii) for all $\{k, n\} \subset \mathbb{Z}, S_{k+p, n+p}=S_{k, n}$.

An SO 1-periodic process is second-order stationary.

REMARKS. (1) By (2.77), it follows that SO $p$-periodic process is WSO $p$-periodic.

(2) By Theorem 2.8, it follows that a $p$-periodic process $x$ such that $\mathbf{E}\|x(k)\|^{2}<+\infty$, $1 \leq k \leq p$, is SO $p$-periodic.

LEMMA 2.20. Let $\{x(n): n \in \mathbb{Z}\}$ be a process in $H$ such that

$$
\forall n \in \mathbb{Z}: \mathbf{E}\|x(n)\|^{2}<+\infty .
$$

The process $\{x(n): n \in \mathbb{Z}\}$ is SO p-periodic if and only if the $H^{p}$-valued process

$$
\vec{x}(n):=(x(n p), x(n p+1), \ldots, x(n p+p-1)), \quad n \in \mathbb{Z}
$$

is SO stationary. 
Proof. We first assume that $\{x(n): n \in \mathbb{Z}\}$ is SO $p$-periodic. Then from condition (i) of Definition 2.19, we have $\mathbf{E} \vec{x}(n)=\mathbf{E} \vec{x}(0), n \in \mathbb{Z}$. Let $\tilde{S}_{n, m}$ be the joint covariance of elements $\vec{x}(n)$ and $\vec{x}(m)$. It can be easily checked that

$$
\tilde{S}_{n, m}=\left(S_{n p+j, m p+l}\right)_{j, l=0}^{p-1}, \quad\{n, m\} \subset \mathbb{Z} .
$$

By condition (ii) of Definition 2.19, it follows that function $\tilde{S}_{n, m}$ is a function of $n-$ $m$ only.

Now assume that $\{\vec{x}(n): n \in \mathbb{Z}\}$ is SO stationary in $H^{p}$. Then the equality

$$
\mathbf{E} \vec{x}(n+1)=\mathbf{E} \vec{x}(n), \quad n \in \mathbb{Z}
$$

implies (i) from Definition 2.19. Since $\tilde{S}_{n, m}$ is a function only of $n-m$, for $S_{n p+j, m p+l}$, we have

$$
\left(u, S_{n p+j, m p+l} v\right)=\mathbf{E}((x(n p+j), u) \overline{(x(m p+l), v)})=\left(\vec{u}, \tilde{S}_{n-m, 0} \vec{v}\right),
$$

where $\vec{u}=\left(u \delta_{j, k}\right)_{k=0}^{k=p}$ and $\vec{v}=\left(v \delta_{l, k}\right)_{k=0}^{k=p}$.

THEOREM 2.21. An $H$-valued process $\{x(n): n \in \mathbb{Z}\}$ satisfying

$$
\mathbf{E}|| x(n) \|^{2}<+\infty, \quad n \in \mathbb{Z}
$$

is SO $p$-periodic, if and only if the $\mathbb{C}^{2}$-valued process

$$
\{((x(n), u),(x(n), v)): n \in \mathbb{Z}\}
$$

is SO p-periodic for every $\{u, v\} \subset H$.

Proof. First, suppose that $\{x(n): n \in \mathbb{Z}\}$ is SO $p$-periodic. It is obvious that

$$
\mathrm{E}((x(n+p), u),(x(n+p), v))=\mathbf{E}((x(n), u),(x(n), v)), \quad n \in \mathbb{Z} .
$$

It can be verified that the joint covariance of vectors

$$
((x(k), u),(x(k), v)), \quad((x(n), u),(x(n), v))
$$

is the matrix

$$
\left(\sigma_{p, q}(k, n)\right)_{p, q=1}^{2}
$$

where

$$
\begin{gathered}
\sigma_{1,1}=\left(u, S_{k, n} u\right), \quad \sigma_{1,2}=\left(u, S_{k, n} v\right)=\bar{\sigma}_{2,1}, \quad \sigma_{2,2}=\left(v, S_{k, n} v\right) ; \\
\sigma_{p, q}(k+p, n+p)=\sigma_{p, q}(k, n), \quad p, q=1,2,\{k, n\} \subset \mathbb{Z} .
\end{gathered}
$$

Now let process (2.92) be SO $p$-periodic. Then

$$
\forall n \in \mathbb{Z} \forall u \in H: \mathrm{E}(x(n+p), u)=\mathrm{E}(x(n), u),
$$


so that

$$
\forall n \in \mathbb{Z}, \forall u \in H:(\mathbf{E} x(n+p)-\mathbf{E} x(n), u)=0 .
$$

Therefore,

$$
\forall n \in \mathbb{Z}: \mathbf{E} x(n+p)=\mathbf{E} x(n) .
$$

Then, using equality

$$
\sigma_{1,2}(k+p, n+p)=\sigma_{1,2}(k, n), \quad\{u, v\} \subset H,
$$

we see that

$$
S_{k+p, n+p}=S_{k, n}, \quad\{k, n\} \subset \mathbb{Z} .
$$

COROLlary 2.22. Let $\{x(n): n \in \mathbb{Z}\}$ be SO $p$-periodic process in $H$ and

$$
m \in \mathbb{N}, \quad\left\{A_{1}, A_{2}, \ldots, A_{m}\right\} \subset \mathscr{L}(H), \quad\left\{n_{1}, n_{2}, \ldots, n_{m}\right\} \subset \mathbb{Z} .
$$

Then the process in $H$

$$
\left\{y(n):=\sum_{j=1}^{m} A_{j} x\left(n+n_{j}\right): n \in \mathbb{Z}\right\}
$$

is SO p-periodic.

Proof. It is simple. The joint covariance of the elements $y(k)$ and $y(n)$ is the operator

$$
\sum_{p, q=1}^{m} A_{p} S_{k+n_{p}, n+n_{q}} A_{q}^{*} .
$$

THEOREM 2.23. Let $\left\{x_{t}(n): n \in \mathbb{Z}\right\}, t \geq 1$, be a sequence of $H$-valued SO $p$-periodic processes and $\{x(n): n \in \mathbb{Z}\}$ be a process in $H$ such that

$$
\forall n \in \mathbb{Z}: \mathbf{E}\left\|x_{t}(n)-x(n)\right\|^{2} \longrightarrow 0, \quad t \rightarrow \infty .
$$

Then the process $\{x(n): n \in \mathbb{Z}\}$ is SO p-periodic.

PROof. It is enough to prove that, for every $n \in \mathbb{Z}$,

$$
\begin{aligned}
& \mathrm{E} x_{t}(n) \rightarrow \mathrm{E} x(n), \quad t \rightarrow \infty, \\
& \mathrm{E}\left(\left(x_{t}(k)-\mathrm{E} x_{t}(k), u\right), \overline{\left(x_{t}(n)-\mathrm{E} x_{t}(n), v\right)}\right) \\
& \quad \rightarrow \mathrm{E}((x(k)-\mathrm{E} x(k), u) \overline{(x(n)-\mathrm{E} x(n), v)}), \quad t \rightarrow \infty ;\{u, v\} \subset H .
\end{aligned}
$$

From the Cauchy inequality, this follows in an obvious way.

REMARK 2.24. About the harmonic analysis of WSO stationary and WSO periodic processes in $H$, see [9, Chapter 2, Section 2.4]. The covariance of a Gaussian stationary Markov process in $H$ is also described in [9, Chapter 2, Section 2.7]. 
3. Stationary and periodic solutions of difference equations. This section contains the conditions for existence of stationary or periodic in distribution solutions to the linear difference equations in the spectral terms. It is proved that these conditions are stable under perturbations of input random process and under bounded perturbation of operator coefficients. The equations with unbounded operator coefficient and nonlinear equations are also considered.

3.1. Linear difference equations. A stochastic process with discrete time in a complex separable Banach space $B$ by definition is a collection of $B$-valued random elements $\{x(n): n \in \mathbb{Z}\}$ defined on a complete probability space $(\Omega, \mathscr{F}, \mathbf{P})$.

Two processes $\{x(n): n \in \mathbb{Z}\}$ and $\{y(n): n \in \mathbb{Z}\}$ are said to be stochastic equivalent if

$$
\forall n \in \mathbb{Z}: \mathbf{P}[x(n)=y(n)]=1 .
$$

Two processes which are stochastic equivalent are in fact almost surely equal. Further, we shall consider equivalent processes as equal.

THEOREM 3.1 (see [7, 9]). Let $A \in \mathscr{L}(B)$. The following statements are equivalent:

(i) the equation

$$
x(n+1)=A x(n)+y(n), \quad n \in \mathbb{Z}
$$

has a unique stationary solution $\{x(n): n \in \mathbb{Z}\}$ in $B$ with $\mathbf{E}\|x(0)\|<+\infty$ for every stationary process $\{y(n): n \in \mathbb{Z}\}$ in $B$ with $\mathbf{E}\|y(0)\|<+\infty$;

(ii) $\sigma(A) \cap S=\varnothing$.

Proof. (i) implies (ii). Let $z \in B, z \neq 0$, and $t \in S$ be fixed. Let $\theta$ be the random variable with the uniform distribution over $[0,2 \pi]$. The process

$$
\left\{y(n):=-z e^{i \theta} t^{n}: n \in \mathbb{Z}\right\}
$$

is stationary in $B$. Let $\{x(n): n \in \mathbb{Z}\}$ be a unique solution for (3.2) corresponds to (3.3). By Hahn-Banach theorem, there is an element $f \in B^{*}$ such that $\langle f, z\rangle=1$. Then, by (3.2),

$$
t^{n} e^{i \theta}=\langle f, A x(n)-x(n+1)\rangle, \quad n \in \mathbb{Z},
$$

therefore the $B \times \mathbb{C}$-valued process

$$
\left\{\left(x(n), e^{i \theta} t^{n}\right): n \in \mathbb{Z}\right\}
$$

is stationary. Hence the process

$$
\left\{x(n) t^{-n} e^{-i \theta}: n \in \mathbb{Z}\right\}
$$

is also stationary, moreover,

$$
\mathbf{E}|| x(n) t^{-n} e^{-i \theta}\|=\mathbf{E}|| x(0)\|<+\infty .
$$


Put

$$
u:=\mathbf{E}\left(x(n) t^{-n} e^{-i \theta}\right), \quad n \in \mathbb{Z} .
$$

Since, by (3.2),

$$
x(n+1) t^{-n} e^{-i \theta}=A x(n) t^{-n} e^{-i \theta}-z, \quad n \in \mathbb{Z},
$$

we have

$$
t u=A u-z
$$

Therefore, for every $z \in B$, (3.10) has the solution $u \in B$. This solution is unique. Indeed, if $v \in B, v \neq u$ is also a solution for (3.10), then the process

$$
\left\{x(n)+t^{n} e^{i \theta}(u-v): n \in \mathbb{Z}\right\}
$$

is a stationary solution for (3.2) which is not equal to $\{x(n): n \in \mathbb{Z}\}$.

Thus $A-t I$ is a one-to-one mapping on $B$ and $t \in(\mathbb{C} \backslash \sigma(A))$.

(ii) implies (i). Let $\{y(n): n \in \mathbb{Z}\}$ be a stationary process such that $\mathbf{E}\|y(0)\|<+\infty$. Then with notations from Section 1 , we define the random element

$$
x(n):=\sum_{j=0}^{\infty}\left(A P_{-}\right)^{j} y(n-1-j)-\sum_{j=-\infty}^{-1}\left(A P_{+}\right)^{j} y(n-1-j) .
$$

The series for $x(n)$ converges almost surely in $B$-norm since by (ii),

$$
\sum_{j=0}^{\infty}\left\|\left(A P_{-}\right)^{j}\right\|+\sum_{j=-\infty}^{-1}\left\|\left(A P_{+}\right)^{j}\right\|<+\infty
$$

By Theorem 2.12, the process $\{x(n): n \in \mathbb{Z}\}$ is stationary and $\mathbf{E}\|x(0)\|<+\infty$. It can be easily checked, by boundedness of $A$, that this process satisfies (3.2).

To prove uniqueness, we consider two stationary solutions $\{x(n): n \in \mathbb{Z}\}$ and $\{u(n): n \in \mathbb{Z}\}$ of (3.2) such that $\mathbf{E}\|x(0)\|<+\infty, \mathbf{E}\|u(n)\|<+\infty$. Then, for every $n \in \mathbb{Z}$, we have

$$
\begin{aligned}
\mathbf{E}\left\|P_{-}(x(n)-u(n))\right\| & \leq\left\|\left(A P_{-}\right)^{m} \sup _{k \in \mathbb{Z}} \mathbf{E}\right\| P_{-}(x(k)-u(k)) \| \\
& \leq\left\|P_{-}\right\| \mathbf{E}(\|x(0)\|+\|u(0)\|)\left\|\left(A P_{-}\right)^{m}\right\|, \\
\mathbf{E}\left\|P_{+}(x(n)-u(n))\right\| & \leq\left\|P_{+}\right\| \mathbf{E}(\|x(0)\|+\|u(0)\|)\left\|\left(A P_{+}\right)^{-m}\right\|
\end{aligned}
$$

for every $m \geq 1$. Therefore, for every $n \in \mathbb{Z}, x(n)=u(n)$ almost surely.

REMARKS. (1) Theorem 3.1 is not a direct consequence of the deterministic Theorem 1.3 and the moment condition of Theorem 3.1 is essential.

(2) If $\sigma(A) \cap\{z|| z \mid<1\} \neq \varnothing$ and $\sigma(A) \cap\{z|| z \mid>1\} \neq \varnothing$, then the stationary solution of (3.2) is not stable for $n \rightarrow+\infty$ or for $n \rightarrow-\infty$. About the concepts of the stochastic stability see [17, 38]. 
(3) If $\sigma(A) \subset\{z|| z \mid<1\}$ then the stationary solution of (3.2) is stable in the mean for $n \rightarrow+\infty$.

(4) If $\{y(n): n \in \mathbb{Z}\}$ is a sequence of independent identically distributed $B$-valued random elements and $\mathscr{F}_{n}$ is the $\sigma$-algebra generated by $y(k), k \leq n$ for $n \in \mathbb{Z}$, then the stationary solution of (3.2), in remark (3), is anticipative with respect to $\left\{\mathscr{F}_{n}, n \in \mathbb{Z}\right\}$ and, in remark (2), is nonanticipative with respect to $\left\{\mathscr{F}_{n}, n \in \mathbb{Z}\right\}$.

THEOREM 3.2. Let $A \in \mathscr{L}(B)$. The following statements are equivalent:

(i) equation (3.2) has a unique $p$-periodic solution $\{x(n): n \in \mathbb{Z}\}$ such that

$$
\mathbf{E}\|x(k)\|<+\infty, \quad 1 \leq k \leq p
$$

for every p-periodic process $\{y(n): n \in \mathbb{Z}\}$, such that

$$
\mathbf{E}\|y(k)\|<+\infty, \quad 1 \leq k \leq p
$$

(ii) $\sigma(A) \cap S=\varnothing$.

Proof. The proof is analogous to that of Theorem 3.1 and is omitted.

REMARK 3.3. (5) The deterministic Theorem 1.5 for (3.2) is proved by weakly condition then condition (ii) of Theorem 3.2.

Let the operators $A(n) \in \mathscr{L}(B), n \in \mathbb{Z}$, be such that

$$
\forall n \in \mathbb{Z}: A(n+p)=A(n) .
$$

Define

$$
\begin{aligned}
B_{0} & :=A(p-1) A(p-2) \cdots A(1) A(0), \\
B_{1} & :=A(0) A(p-1) \cdots A(2) A(1), \\
& \vdots \\
B_{p-1} & :=A(p-2) A(p-3) \cdots A(0) A(p-1) .
\end{aligned}
$$

THEOREM 3.4. The following statements are equivalent:

(i) the equation

$$
x(n+1)=A(n) x(n)+y(n), \quad n \in \mathbb{Z}
$$

has a unique $p$-periodic solution $\{x(n): n \in \mathbb{Z}\}$, such that

$$
\mathbf{E}\|x(k)\|<+\infty, \quad 1 \leq k \leq p,
$$

for every p-periodic random process $\{y(n): n \in \mathbb{Z}\}$ in $B$, such that

$$
\mathbf{E}\|y(k)\|<+\infty, \quad 1 \leq k \leq p
$$

(ii) there is $k \in\{0,1, \ldots, p-1\}: \sigma\left(B_{k}\right) \cap S=\varnothing$;

(iii) for all $k \in\{0,1, \ldots, p-1\}: \sigma\left(B_{k}\right) \cap S=\varnothing$. 
Proof. (i) implies (iii). Let $\{z(k): k \in \mathbb{Z}\}$ be a $B$-valued stationary process such that $\mathrm{E}\|z(0)\|<+\infty$. Fix $j \in\{1,2, \ldots, p\}$ and define

$$
\begin{gathered}
y(k p+j-1):=z(k-1), \\
y(k p+s):=\overline{0}, \quad s \in\{0,1, \ldots, p-1\} \backslash\{j-1\}
\end{gathered}
$$

for $k \in \mathbb{Z}$. By Lemma 2.5, $\{y(n): n \in \mathbb{Z}\}$ is $p$-periodic in $B$. It is evident that

$$
\mathbf{E}\|y(k)\|<+\infty, \quad 1 \leq k \leq p .
$$

Let $\{x(n): n \in \mathbb{Z}\}$ be a unique $p$-periodic solution for (3.19) with the above constructed process $\{y(k): k \in \mathbb{Z}\}$. By Lemma 2.5 , the process $\{x(k p+j): k \in \mathbb{Z}\}$ is stationary; and it can be easily checked that this process satisfies the equation

$$
x((k+1) p+j)=B_{j} x(k p+j)+z(k), \quad k \in \mathbb{Z}, B_{p}=B_{0} .
$$

By Theorem 3.1, $\sigma\left(B_{j}\right) \cap S=\varnothing$.

(ii) is equivalent to (iii). See Remark 1.13.

(ii) implies (i). Let $\sigma\left(B_{0}\right) \cap S=\varnothing$ and $\{y(n): n \in \mathbb{Z}\}$ be a $p$-periodic process in $B$ such that

$$
\mathrm{E}\|y(k)\|<+\infty, \quad 1 \leq k \leq p
$$

Define

$$
z_{0}(k):=\sum_{t=1}^{p-1} A(p-1) A(p-2) \cdots A(p-t) y((k+1) p-t-1)+y((k+1) p-1), \quad k \in \mathbb{Z} .
$$

By Lemma 2.5, the process $\left\{z_{0}(k): k \in \mathbb{Z}\right\}$ is stationary, moreover, $\mathbf{E}\left\|z_{0}(0)\right\|<+\infty$. By Theorem 3.1, there is a unique stationary $B$-valued process $\left\{x_{0}(k): k \in \mathbb{Z}\right\}$ satisfies the equation

$$
x_{0}(k+1)=B_{0} x_{0}(k)+z_{0}(k), \quad k \in \mathbb{Z} ; \quad \mathbf{E}\left\|x_{0}(0)\right\|<+\infty .
$$

Now, we consider the process $\{x(n): n \in \mathbb{Z}\}$ defined by

$$
\begin{aligned}
x(k p):= & x_{0}(k), \\
x(k p+1):= & A(0) x_{0}(k)+y(k p), \\
x(k p+2):= & A(1) x(k p+1)+y(k p+1) \\
= & A(1) A(0) x_{0}(k)+A(1) y(k p)+y(k p+1), \\
\vdots & \\
x(k p+p-1):= & A(p-2) x(k p+p-2)+y(k p+p-2) \\
= & A(p-2) A(p-3) \cdots A(0) x_{0}(k) \\
& +A(p-2) A(p-3) \cdots A(1) y(k p) \\
& +A(p-2) \cdots A(2) y(k p+1) \\
& +A(p-2) \cdots A(3) y(k p+2) \\
& +\cdots+y(k p+p-2), \quad k \in \mathbb{Z} .
\end{aligned}
$$


It can be easily verified that the $B^{p}$-valued process

$$
\{(x(k p), x(k p+1), \ldots, x(k p+p-1)): n \in \mathbb{Z}\}
$$

is stationary and therefore, by Lemma 2.5 , the process $\{x(n): n \in \mathbb{Z}\}$ is $p$-periodic in $B$. The equality

$$
x(n+1)=A(n) x(n)+y(n),
$$

holds for the value $n=k p+j, k \in \mathbb{Z}, 0 \leq j \leq p-2$, by the definition of process $\{x(n): n \in \mathbb{Z}\}$. To prove (3.30) for values $n=k p+p-1, k \in \mathbb{Z}$, we have

$$
\begin{aligned}
A(p-1) x(k p+p-1)= & B_{0} x_{0}(k)+A(p-1) \cdots A(1) y(k p) \\
& +A(p-1) \cdots A(2) y(k p+1) \\
& +A(p-1) \cdots A(3) y(k p+2) \\
& +\cdots+A(p-1) y(k p+p-2) \\
= & B_{0} x_{0}(k)+z_{0}(k)-y((k+1) p-1) \\
= & x_{0}(k+1)-y((k+1) p-1) .
\end{aligned}
$$

We consider the equation

$$
A x(n)=x(n+1)-2 x(n)+x(n-1)+y(n), \quad n \in \mathbb{Z},
$$

which can be found in various applications, see [26, 32, 35]. The following existence stationary solution theorem is based on some modification of the method used in Section 1 .

Let $A$ be a closed linear operator with dense domain $D(A)$ in $B$. It is known that $D(A) \in \mathscr{B}(B)$ see [29, Chapter 3 , Section IV]. Let $\{y(n): n \in \mathbb{Z}\}$ be a random process.

DEFINITION 3.5. A random process $\{x(n): n \in \mathbb{Z}\}$ is called a solution of (3.32) if, for every $n \in \mathbb{Z}$,

(i) $\mathbf{P}[x(n) \in D(A)]=1$,

(ii) equality (3.32) holds with the probability one.

THEOREM 3.6 (see $[8,20])$. The following statements are equivalent:

(i) there is a sequence of operators $\{c(k): k \in \mathbb{Z}\} \in \mathcal{M}$, and (3.32) has a unique stationary solution $\{x(n): n \in \mathbb{Z}\}$ such that

$$
x(n)=\sum_{k \in \mathbb{Z}} c(n-k) y(k), \quad n \in \mathbb{Z} ; \quad \mathbf{E}\|x(0)\|<+\infty,
$$

for every stationary process in $B\{y(n): n \in \mathbb{Z}\}$ with $\mathbf{E}\|y(0)\|<+\infty$;

(ii) $\sigma(A) \cap[-4,0]=\varnothing$.

Proof. (i) implies (ii). By definition of the family $\mathcal{M}$, the series

$$
\sum_{k \in \mathbb{Z}} c(n-k) y(k), \quad \sum_{k \in \mathbb{Z}} A c(n-k) y(k)
$$


converges almost surely in $B$-norm for every $n \in \mathbb{Z}$. By the closedness of operator $A$, we have

$$
A x(n)=\sum_{k \in \mathbb{Z}} A c(n-k) y(k), \quad n \in \mathbb{Z} .
$$

Hence, (3.32) implies that

$$
\sum_{k \in \mathbb{Z}} U(k) y(k)=\overline{0}
$$

where

$$
U(k):=A c(n-k)-c(n+1-k)+2 c(n-k)-c(n-1-k)-\delta_{n, k} I, \quad k \in \mathbb{Z} .
$$

We observe that $U(k) \in \mathscr{L}(B), k \in \mathbb{Z}$. Now let $\{y(n): n \in \mathbb{Z}\}$ be a sequence of independent identically distributed random elements such that $\mathbf{E} y(0)=\overline{0}$. Fixing $n \in \mathbb{Z}$ and taking the conditional expectation of (3.36) gives the $\sigma$-algebra generated by $y(k)$, $k \neq n$, we have

$$
U(n) y(n)=\overline{0}
$$

Hence, it follows that $U(n)=\Theta$. Indeed, let $\{u(j): j \geq 1\}$ be a countable dense set in $B$. The random element $y(n)$, by definition, is such that

$$
\begin{gathered}
\mathbf{P}[y(n)=u(j)]=\left(2^{j+1}(1+\|u(j)\|)\right)^{-1}, \quad j \geq 1 ; \\
\mathbf{P}[y(n)=\overline{0}]=1-\sum_{j=1}^{\infty}\left(2^{j+1}(1+\|u(j)\|)\right)^{-1} .
\end{gathered}
$$

Then $U(n) u(j)=\overline{0}, j \geq 1$. Therefore, $U(n)=\Theta$.

Thus we have

$$
A c(n-k)=c(n+1-k)-2 c(n-k)+c(n-1-k)+\delta_{n, k} I .
$$

Let

$$
\Phi_{-}(z):=\sum_{k \in \mathbb{Z}} c(k) z^{k}, \quad z \in K:=\left\{z \in \mathbb{C}\left|t_{0} \leq\right| z \mid \leq t_{1}\right\} ; \Phi_{-}(z) \in \mathscr{L}(B),
$$

where $t_{0}, t_{1}$ are the numbers corresponding to the sequence $\{c(k): k \in \mathbb{Z}\}$ in the definition of $\mathcal{M}$, see Section 1 . By the definition of $\mathcal{M}$, the series

$$
\sum_{k \in \mathbb{Z}} A c(k) z^{k}, \quad z \in K
$$

also converges in the operator norm; and by the closedness of $A$, it follows that

$$
\Phi_{-}(z) x \in D(A), \quad A \Phi_{-}(z)=\sum_{k \in \mathbb{Z}} A c(k) z^{k},
$$

for all $x \in B$. Now, by (3.40), we obtain

$$
\left(A-\left(\frac{1}{z}-2+z\right) I\right) \Phi_{-}(z)=I, \quad z \in K .
$$


Therefore, for every $y \in B, z \in K$, the equation

$$
\left(A-\left(\frac{1}{z}-2+z\right) I\right) x=y
$$

has the solution $x:=\Phi_{-}(z) y \in D(A)$, moreover,

$$
\|x\| \leq \sum_{k \in \mathbb{Z}}\|c(k)\| \cdot\|y(k)\| .
$$

For $z \in K$, fixed (3.45) has a unique solution $x$ for every $y \in B$. Indeed, if a solution of (3.45) is not unique, then there is $u \in D(A), u \neq \overline{0}$, such that

$$
\left(A-\left(\frac{1}{z}-2+z\right) I\right) u=\overline{0}
$$

Now put $y=u$ in (3.45). Then we find

$$
u=\left(A-\left(\frac{1}{z}-2+z\right) I\right) \Phi_{-}(z) u=\Phi_{-}(z)\left(A-\left(\frac{1}{z}-2+z\right) I\right) u=\overline{0},
$$

a contradiction. Thus $1 / z-2+z$ lies in the resolvent set of operator $A$. It can be easily checked that

$$
[-4,0] \subset\left\{\frac{1}{z}-2+z: z \in K\right\} .
$$

We note that function $\Phi_{-}$is uniquely defined by (3.45) and the sequence $\{c(k): k \in \mathbb{Z}\}$ is uniquely defined by the Laurent series of $\Phi_{-}$.

(ii) implies (i). Let $\sigma(A) \cap[-4,0]=\varnothing$. Since the set $\rho(A):=\mathbb{C} \backslash \sigma(A)$ is an open set, there are numbers $t_{0}$ and $t_{1}$ such that $0<t_{0}<1<t_{1},(1 / z-2+z) \in \rho(A), z \in K$. For $z \in K$, put

$$
\Phi_{-}(z):=\left(A-\left(\frac{1}{z}-2+z\right) I\right)^{-1} .
$$

The operator-valued function $\Phi_{-}$is analytic in $K$; and for every $x \in B$, the relation $\Phi_{-}(z) x \in D(A)$ is true. Hence, the following expansion in Laurent series

$$
\Phi_{-}(z)=\sum_{k \in \mathbb{Z}} c(k) z^{k}, \quad z \in K
$$

is valid, where

$$
c(k)=\frac{1}{2 \pi i} \oint_{|s|=1} \Phi_{-}(s) s^{-k-1} d s, \quad c(k) \in \mathscr{L}(B), k \in \mathbb{Z} .
$$

This series absolutely converges in $K$ in operator norm. By definition of $\Phi_{-}$,

$$
\left(A-\left(\frac{1}{z}-2+z\right) I\right) \Phi_{-}(z)=I, \quad z \in K
$$

and therefore (3.40) is true. Moreover,

$$
A \Phi_{-}(z)=\left(\frac{1}{z}-2+z\right) \Phi_{-}(z)+I, \quad z \in K .
$$


Hence, the function $A \Phi_{-}$also is analytic in $K$; and by the closedness of $A$, we have for every $k \in \mathbb{Z}$,

$$
A c(k)=\frac{1}{2 \pi i} \oint_{|s|=1} A \Phi_{-}(s) s^{-k-1} d s .
$$

Thus, $\{c(k): k \in \mathbb{Z}\} \in \mathcal{M}$ and

$$
A \Phi_{-}(z)=\sum_{k \in \mathbb{Z}} A c(k) z^{k}, \quad z \in K .
$$

By the relation $\{c(k): k \in \mathbb{Z}\} \in \mathcal{M}$, the series

$$
\sum_{k \in \mathbb{Z}} c(n-k) y(k), \quad \sum_{k \in \mathbb{Z}} A c(n-k) y(k)
$$

converge almost surely in $B$-norm. Since operator $A$ is closed, it follows that

$$
x(n) \in D(A), \quad A x(n)=\sum_{k \in \mathbb{Z}} A c(n-k) y(k)
$$

for every $n \in \mathbb{Z}$. By Theorem 2.12, process $\{x(n): n \in \mathbb{Z}\}$ is stationary. It can be easily verified that relations (3.40) imply (3.32). Uniqueness is obvious.

We now take up an important extension of Theorem 3.1. Suppose that $\omega$ is a function which satisfies Condition 1.6 from Section 1 and let $\left\{a_{k}: k \in \mathbb{Z}\right\}$ be the Laurent series coefficients for $\omega$.

THEOREM 3.7 (see [20]). Let A be a closed operator. The following statements are equivalent:

(i) there is a sequence of operators $\{c(k): k \in \mathbb{Z}\} \in \mathcal{M}$; and the equation

$$
\sum_{k \in \mathbb{Z}} a_{k} x(n+k)=A x(n)+y(n), \quad n \in \mathbb{Z}
$$

has a unique stationary solution $\{x(n): n \in \mathbb{Z}\}$ such that

$$
x(n)=\sum_{k \in \mathbb{Z}} c(n-k) y(k), \quad n \in \mathbb{Z} ; \quad \mathbf{E}\|x(0)\|<+\infty,
$$

for every stationary process in $B\{y(n): n \in \mathbb{Z}\}$ with $\mathbf{E}\|y(0)\|<+\infty$;

(ii) $\sigma(A) \cap \omega(S)=\varnothing$.

Proof. The proof is analogous to that of Theorem 3.6 and is omitted. See also the deterministic Theorem 1.8 for comparison.

Just in the same way, we also get the following result.

THEOREM 3.8 (see [9]). Let $A$ be a closed operator. The following statements are equivalent:

(i) there is a sequence of operators $\{c(k): k \in \mathbb{Z}\} \in M$; and (3.59) has a unique $p$-periodic solution $\{x(n): n \in \mathbb{Z}\}$ such that

$$
x(n)=\sum_{k \in \mathbb{Z}} c(n-k) y(k), \quad n \in \mathbb{Z} ; \quad \mathbf{E}\|x(k)\|<+\infty, \quad 1 \leq k \leq p,
$$

for every $p$-periodic process in $B\{y(n): n \in \mathbb{Z}\}$ with $\mathrm{E}\|y(k)\|<+\infty, 1 \leq k \leq p$; 
(ii) $\sigma(A) \cap \omega(S)=\varnothing$.

TheOREM 3.9 (see [9]). Let

$$
p \in(\mathbb{N} \cup\{0\}), \quad\left\{A_{0}, A_{1}, \ldots, A_{p}\right\} \subset \mathscr{L}(B) .
$$

The following statements are equivalent:

(i) the equation

$$
A_{0} x(n)+A_{1} x(n-1)+\cdots+A_{p}(n-p)=y(n), \quad n \in \mathbb{Z}
$$

has a unique stationary solution $\{x(n): n \in \mathbb{Z}\}$ such that $\mathbf{E}\|x(0)\|<+\infty$ for every stationary process $\{y(n): n \in \mathbb{Z}\}$ with $\mathbf{E}\|y(0)\|<+\infty$;

(ii) for every $\lambda \in S$, the operator

$$
A_{0} \lambda^{p}+A_{1} \lambda^{p-1}+\cdots+A_{p}
$$

has a bounded inverse.

Proof. (i) implies (ii). It can be established by the use of an argument similar to that of the first part of Theorem 3.1.

(ii) implies (i). Suppose that, for every $\lambda \in S$, operator (3.64) has an inverse defined on $B$. Then, for every $\lambda \in S$, there is $r(\lambda)>0$ such that the operator

$$
A_{0} z^{p}+A_{1} z^{p-1}+\cdots+A_{p}
$$

has a bounded inverse for all $z$ with $|z-\lambda|<r(\lambda)$. Since $S$ is compact in $\mathbb{C}$, there is an annulus

$$
K=\left\{z \in \mathbb{C}\left|t_{0}<\right| z \mid<t_{1}\right\}, \quad 0<t_{0}<1<t_{1}<2,
$$

such that, for every $z \in K$, operator (3.65) has bounded inverse $R(z)$. Now we prove that the function $R$ is analytic in $K$. Let $z_{0} \in K$ be a fixed point. By definition of $R$, it follows that

$$
\begin{aligned}
R(z)= & R(z)\left(A_{0} z_{0}^{p}+A_{1} z_{0}^{p-1}+\cdots+A_{p}\right) R\left(z_{0}\right) \\
= & R(z)\left(A_{0} z^{p}+A_{1} z^{p-1}+\cdots+A_{p}-A_{0}\left(z^{p}-z_{0}^{p}\right)\right. \\
& \left.-A_{1}\left(z^{p-1}-z_{0}^{p-1}\right)-\cdots-A_{p-1}\left(z-z_{0}\right)\right) R\left(z_{0}\right) \\
= & R\left(z_{0}\right)-R(z) \sum_{j=0}^{p-1} A_{j}\left(z^{p-j}-z_{0}^{p-j}\right) R\left(z_{0}\right) .
\end{aligned}
$$

Therefore,

$$
R(z)\left(I+\sum_{j=0}^{p-1} A_{j} R\left(z_{0}\right)\left(z^{p-j}-z_{0}^{p-j}\right)\right)=R\left(z_{0}\right) .
$$

By equality (3.68), we obtain

$$
R(z)=R\left(z_{0}\right) \sum_{n=0}^{\infty}\left(\sum_{j=0}^{p-1} A_{j} R\left(z_{0}\right)\left(z_{0}^{p-j}-z^{p-j}\right)\right)^{n},
$$


for every value $z$, such that

$$
\left|z-z_{0}\right| \sum_{j=0}^{p-1}\left\|A_{j} R\left(z_{0}\right)\right\| 2^{2(p-j)}<1 .
$$

Hence

$$
\begin{gathered}
R(z)=R\left(z_{0}\right) \sum_{n=0}^{\infty}\left(-\sum_{j=0}^{p-1} A_{j} R\left(z_{0}\right) \sum_{k=1}^{p-j} C_{p-j}^{k} z_{0}^{p-j-k}\left(z-z_{0}\right)^{k}\right)^{n}, \\
\sum_{n=0}^{\infty}\left(\sum_{j=0}^{p-1}\left\|A_{j} R\left(z_{0}\right)\right\| \sum_{k=1}^{p-j} C_{p-j}^{k} 2^{p-j-k}\left|z-z_{0}\right|^{k}\right)^{n}<+\infty
\end{gathered}
$$

if, in addition, $\left|z-z_{0}\right|<1$. Let

$$
R(z)=\sum_{k \in \mathbb{Z}} c(k) z^{k}, \quad z \in K
$$

be the Laurent series for $R$. Note that

$$
\begin{gathered}
c(k):=\frac{1}{2 \pi i} \oint_{S} R(z) z^{-k-1} d z, \quad c(k) \in \mathscr{L}(B), k \in \mathbb{Z}, \\
\sum_{k \in \mathbb{Z}}\|c(k)\|<+\infty .
\end{gathered}
$$

Suppose that $\{y(n): n \in \mathbb{Z}\}$ is a stationary process with $\mathbf{E}\|y(0)\|<+\infty$ and put

$$
x(n):=\sum_{k \in \mathbb{Z}} c(k-n-p) y(k), \quad k \in \mathbb{Z} .
$$

By (3.74), the series for $x(n)$ converges almost surely in the $B$-norm. By Theorem 2.12, the process $\{x(n): n \in \mathbb{Z}\}$ is stationary. The process $\{x(n): n \in \mathbb{Z}\}$ satisfies (3.63). To establish this, note that for every $n \in \mathbb{Z}$, we have

$$
\begin{aligned}
A_{0} x(n) & +A_{1} x(n-1)+\cdots+A_{p} x(n-p) \\
& =\sum_{k \in \mathbb{Z}}\left(A_{0} c(k-n-p)+A_{1} c(k-n-p+1)+\cdots+A_{p} c(k-n)\right) y(k) .
\end{aligned}
$$

By (3.72) and the identity

$$
\left(A_{0} z^{p}+A_{1} z^{p-1}+\cdots+A_{p}\right) R(z)=I, \quad z \in K,
$$

we have

$$
\begin{aligned}
& \sum_{k \in \mathbb{Z}}\left(A_{0} c(k) z^{k+p}+A_{1} c(k) z^{k+p-1}+\cdots+A_{p} c(k) z^{k}\right) \\
&=\sum_{k \in \mathbb{Z}}\left(A_{0} c(k-p)+A_{1} c(k-p+1)+\cdots+A_{p} c(k)\right) z^{k}=I .
\end{aligned}
$$

By uniqueness expansion in a Laurent series, it follows that

$$
\begin{aligned}
A_{0} c(-p)+A_{1} c(-p+1)+\cdots+A_{p} c(0) & =I, \\
A_{0} c(k-p)+A_{1} c(k-p+1)+\cdots+A_{p} c(k) & =\Theta, \quad k \neq 0 .
\end{aligned}
$$

From this and (3.76), (3.63) follows. 
Now we prove uniqueness. Let $\{u(n): n \in \mathbb{Z}\}$ be a stationary solution of (3.63) with $\mathrm{E}\|u(0)\|<+\infty$ and

$$
w(n):=x(n)-u(n), \quad n \in \mathbb{Z} .
$$

Then

$$
A_{0} w(n)+A_{1} w(n-1)+\cdots+A_{p} w(n-p)=\overline{0}, \quad n \in \mathbb{Z} .
$$

By (3.79) for the process

$$
v(n):=\sum_{k \in \mathbb{Z}} c(k-n-p) w(k), \quad n \in \mathbb{Z},
$$

we have

$$
A_{0} v(n)+A_{1} v(n-1)+\cdots+A_{p} v(n-p)=w(n), \quad n \in \mathbb{Z} .
$$

In addition, by identity

$$
\left(A_{0} z^{p}+A_{1} z^{p-1}+\cdots+A_{p}\right) R(z)=R(z)\left(A_{0} z^{p}+A_{1} z^{p-1}+\cdots+A_{p}\right), \quad z \in K,
$$

it follows that

$$
\begin{aligned}
& A_{0} c(k-p)+A_{1} c(k-p+1)+\cdots+A_{p} c(k) \\
& \quad=c(k-p) A_{0}+c(k-p+1) A_{1}+\cdots+c(k) A_{p}, \quad k \in \mathbb{Z} .
\end{aligned}
$$

Therefore,

$$
\begin{array}{rl}
A_{0} & v(n)+A_{1} v(n-1)+\cdots+A_{p} v(n-p) \\
& =\sum_{k \in \mathbb{Z}}\left(A_{0} c(k-n-p)+A_{1} c(k-n-p+1)+\cdots+A_{p} c(k-n)\right) w(n) \\
& =\sum_{k \in \mathbb{Z}}\left(c(k-n-p) A_{0}+c(k-n-p+1) A_{1}+\cdots+c(k-n) A_{p}\right) w(n) \\
& =\sum_{k \in \mathbb{Z}} c(k-n-p)\left(A_{0} w(n)+A_{1} w(n-1)+\cdots+A_{p} w(n-p)\right)=\overline{0}, \quad n \in \mathbb{Z} .
\end{array}
$$

Stationary solutions of a two-dimensional stochastic difference equation in a Banach space have been developed in [19].

3.2. Perturbed equation. In many problems of applications, a given system is subject to deterministic and random influences that must be taken into account in the appropriate mathematical models. Usually, mathematical models are only approximation to real behavior of systems. Therefore, mathematical models must be stable under perturbation of its parameters. From the viewpoint of applications, stability is just as important as existence. We give such results in this section. From the mathematical viewpoint, many of such results are the theorems about the continuous dependence of solution in given norm from equation coefficients. 
THEOREM 3.10 (see $[11,12])$. Let the operators

$$
A \in \mathscr{L}(B), \quad\left\{A_{m}(n), n \in \mathbb{Z}, m \geq 1\right\} \subset \mathscr{L}(B)
$$

satisfy the following conditions:

(i) $\sigma(A) \cap S=\varnothing$;

(ii) $\delta_{m}:=\sup \left\{\left\|A_{m}(n)-A\right\| \mid n \in \mathbb{Z}\right\} \rightarrow 0, m \rightarrow \infty$.

Then for each stationary $B$-valued process $\{y(n): n \in \mathbb{Z}\}$ with $\mathbf{E}\|y(0)\|<+\infty$, we have the equation

$$
x(n+1)=A x(n)+y(n), \quad n \in \mathbb{Z}
$$

and for every $m$ greater than some $m_{0} \in \mathbb{N}$, the equation

$$
x_{m}(n+1)=A_{m}(n) x_{m}(n)+y(n), \quad n \in \mathbb{Z}
$$

has a unique stationary solution $\{x(n): n \in \mathbb{Z}\}$ and a unique solution $\left\{x_{m}(n): n \in \mathbb{Z}\right\}$, respectively, for which

$$
\begin{gathered}
\mathbf{E}\|x(0)\|<+\infty ; \quad \sup _{n \in \mathbb{Z}} \mathbf{E}\left\|x_{m}(n)\right\|<+\infty, \quad n \in \mathbb{Z}, \\
\sup _{n \in \mathbb{Z}} \mathrm{E}\left\|x_{m}(n)-x(n)\right\| \longrightarrow 0, \quad m \longrightarrow \infty .
\end{gathered}
$$

Proof. By Theorem 3.1, it follows that condition (i) is equivalent to the existence of a unique stationary solution $\{x(n): n \in \mathbb{Z}\}$ with $\mathbf{E}\|x(0)\|<+\infty$ of (3.88) for every stationary process $\{y(n): n \in \mathbb{Z}\}$ with $\mathbf{E}\|y(0)\|<+\infty$. Moreover, as in Theorem 3.1, let

$$
\sigma_{-}(A):=\sigma(A) \cap\{z \in \mathbb{C}|| z \mid<1\}, \quad \sigma_{+}(A):=\sigma(A) \backslash \sigma_{-}(A),
$$

and let $P_{-}$and $P_{+}$be the spectral projectors corresponding to the spectral sets $\sigma_{-}(A)$ and $\sigma_{+}(A)$, respectively. Then, for every $n \in \mathbb{Z}$, we have

$$
x(n)=\sum_{j \in \mathbb{Z}} G(j) y(n-j-1),
$$

where, for $j \in \mathbb{Z}$,

$$
G(j):= \begin{cases}\left(A P_{-}\right)^{j}, & j \geq 0, \\ -\left(A P_{+}\right)^{j}, & j \leq-1 .\end{cases}
$$

The series for $x(n)$ converges almost surely in $B$-norm and $\mathbf{E}\|x(0)\|<+\infty$, moreover,

$$
L:=\sum_{j \in \mathbb{Z}}\|G(j)\|<+\infty .
$$

Let $m_{0} \in \mathbb{Z}$ be such that $L \delta_{m}<1$ for $m>m_{0}$. Now let $m>m_{0}$. We prove the existence of solution for (3.89) by showing that the sequence $\left\{x_{m}^{j} \mid j \geq 1\right\}$, defined by

$$
\begin{gathered}
x_{m}^{0}(n):=\overline{0}, \quad n \in \mathbb{Z}, \\
x_{m}^{j+1}(n+1)=A x_{m}^{j+1}(n)+\left(A_{m}(n)-A\right) x_{m}^{j}(n)+y(n), \quad n \in \mathbb{Z}, j \geq 0,
\end{gathered}
$$


converges as $j \rightarrow \infty$ to a solution of (3.89). First, we have

$$
\Delta_{m}^{j} \leq L \delta_{m} \Delta_{m}^{j-1}, \quad j \geq 1
$$

for

$$
\Delta_{m}^{j}:=\sup _{n \in \mathbb{Z}} \mathbf{E}\left\|x_{m}^{j+1}(n)-x_{m}^{j}(n)\right\| .
$$

Then by [27], for every $n \in \mathbb{Z}$, there is a random element $x_{m}(n)$ such that

$$
x_{m}^{j}(n) \longrightarrow x_{m}(n), \quad j \rightarrow \infty
$$

almost surely in B-norm. In addition,

$$
\sup _{m \geq 1} \sup _{n \in \mathbb{Z}} \mathbf{E}\left\|x_{m}(n)\right\|<+\infty
$$

and taking the limit in $j$ in both sides of the equality (3.96), we obtain that the process $\left\{x_{m}(n): n \in \mathbb{Z}\right\}$ satisfies (3.89). From (3.88) and (3.89), it follows that

$$
\begin{aligned}
& \sup _{n \in \mathbb{Z}} \mathbf{E}\left\|x(n)-x_{m}(n)\right\| \leq L \delta_{m} \mathbf{E}\left\|x_{m}(n)\right\|, \\
& \sup _{n \in \mathbb{Z}} \mathbf{E}\left\|x(n)-x_{m}(n)\right\| \leq \frac{L \delta_{m}}{1-L \delta_{m}} \mathbf{E}\|y(0)\| .
\end{aligned}
$$

REMARK 3.11. Theorem 3.10 may be generalized to the more general case of perturbations. Let

$$
\begin{gathered}
\left\{A_{m}^{v}(n) \mid n \in \mathbb{Z}, v \geq 0, m \geq 1\right\} \subset \mathscr{L}(B), \\
\delta_{m}^{\prime}:=\sup _{n \in \mathbb{Z}}\left\|A_{m}^{0}(n)-A\right\|+\sum_{v=1}^{\infty} \sup _{n \in \mathbb{Z}}\left\|A_{m}^{v}(n)\right\| \longrightarrow 0, \quad m \longrightarrow \infty .
\end{gathered}
$$

Then, the statement of Theorem 3.10 holds for the solutions of the following equations:

$$
x_{m}(n+1)=\sum_{v=0}^{\infty} A_{m}^{v}(n) x_{m}(n-v)+y(n), \quad n \in \mathbb{Z} ; m \geq 1 .
$$

We now return to the linear stochastic equation (3.19). Let $\{A(n): n \in \mathbb{Z}\} \subset \mathscr{L}(B)$; and, for a fixed $p \in \mathbb{N}$, let

$$
A(n+p)=A(n), \quad n \in \mathbb{Z} .
$$

Put $B_{0}:=A(p-1) A(p-2) \cdots A(1) A(0)$.

Condition 3.12. The operators $\left\{A(n), A_{m}(n), m \geq 1 ; n \in \mathbb{Z}\right\} \subset \mathscr{L}(B)$ satisfy the following conditions:

(i) $\sigma\left(B_{0}\right) \cap S=\varnothing$;

(ii) $\sup _{n \in \mathbb{Z}}\left\|A_{m}(n)-A(n)\right\| \rightarrow 0, m \rightarrow \infty$.

THEOREM 3.13. Let Condition 3.12 hold and let $\{y(n): n \in \mathbb{Z}\}$ be a $p$-periodic in distribution process with $\mathbf{E}\|y(k)\|<+\infty, k=1,2, \ldots, p$. 
Then, the equation

$$
x(n+1)=A(n) x(n)+y(n), \quad n \in \mathbb{Z}
$$

and, for every $m$ greater than some $m_{0} \in \mathbb{N}$, the equation

$$
x_{m}(n+1)=A_{m}(n) x_{m}(n)+y(n), \quad n \in \mathbb{Z}
$$

have a unique $p$-periodic in distribution solution $\{x(n): n \in \mathbb{Z}\}$, and a unique solution $\left\{x_{m}(n): n \in \mathbb{Z}\right\}$, respectively, for which

$$
\begin{gathered}
\mathrm{E}\|x(k)\|<+\infty, \quad k=1,2, \ldots, p ; \quad \sup _{n \in \mathbb{Z}} \mathrm{E}\left\|x_{m}(n)\right\|<+\infty ; \\
\sup _{n \in \mathbb{Z}} \mathrm{E}\left\|x_{m}(n)-x(n)\right\| \longrightarrow 0, \quad m \longrightarrow \infty .
\end{gathered}
$$

Proof. The proof of Theorem 3.13 is similar to that of Theorem 3.10 and we give only new features. First notice that, for each process $\{y(n): n \in \mathbb{Z}\}$ with

$$
\sup _{n \in \mathbb{Z}} \mathrm{E}\|y(n)\|<+\infty
$$

equation (3.88), under condition (i) of Theorem 3.10, has a unique solution $\{x(n)$ : $n \in \mathbb{Z}\}$ with

$$
\sup _{n \in \mathbb{Z}} \mathrm{E}\|x(n)\|<+\infty .
$$

The proof of this statement follows along the lines of the proof of Theorem 3.10.

It is easily seen that the solution $\{x(n): n \in \mathbb{Z}\}$ for (3.105) satisfies the equation

$$
x((v+1) p)=B_{0} x(v p)+z(v), \quad v \in \mathbb{Z}
$$

with

$z(v):=\sum_{t=1}^{p-1} A(p-1) A(p-2) \cdots A(p-t) y((v+1) p-t-1)+y((v+1) p-1), \quad v \in \mathbb{Z}$.

Then, using the above statement, we define $\{x(\nu p): \nu \in \mathbb{Z}\}$ as the solution for (3.110) and put

$$
\begin{aligned}
x(v p+1) & :=A(0) x(v p)+y(v p), \\
x(v p+2) & :=A(1) x(v p+1)+y(v p+1), \\
& \vdots \\
x(v p+p-1) & :=A(p-2) x(v p+p-2)+y(v p+p-2),
\end{aligned}
$$

we have the solution $\{x(n): n \in \mathbb{Z}\}$ for (3.105).

Now, using the approximating method of Theorem 3.10, it is easy to prove the existence of a solution for (3.106) for every $m$ greater than some $m_{0} \in \mathbb{N}$.

The study of a random perturbation is complicated and more interesting. In the sequel, we investigate this question. The following result will be useful in applications. 
Theorem 3.14 (see [5, Chapter 10, Section 1], [9]). Let $\{A, C\} \subset \mathscr{L}(B)$ and let $\{(\varepsilon(n)$, $y(n)): n \in \mathbb{Z}\}$ be a stationary metrically transitive random process in $\mathbb{C} \times B$. Suppose that

(i) $\operatorname{Eln}^{+}\|y(0)\|<+\infty$;

(ii) $\mu:=\mathrm{E} \ln \|A+\varepsilon(0) C\|<+\infty$.

Then the equation

$$
x(n+1)=(A+\varepsilon(n) C) x(n)+y(n), \quad n \in \mathbb{Z}
$$

has a unique stationary solution $\{x(n): n \in \mathbb{Z}\}$.

Proof. Let $n \in \mathbb{Z}$ be fixed. We show that the series

$$
x(n):=y(n-1)+\sum_{j=1}^{\infty}(A+\varepsilon(n-1) C)(A+\varepsilon(n-2) C) \cdots(A+\varepsilon(n-j) C) y(n-j-1)
$$

converges almost surely in $B$-norm. It is obvious that

$$
\begin{aligned}
(\|(A+\varepsilon(n-1) C) & (A+\varepsilon(n-2) C) \cdots(A+\varepsilon(n-j)) C y(n-j-1) \|)^{1 / j} \\
\leq & \exp \left(\frac{1}{j} \sum_{t=n-j-1}^{n-1} \ln \|A+\varepsilon(t) C\|+\frac{1}{j} \ln ^{+}\|y(n-j-1)\|\right) .
\end{aligned}
$$

Since $\varepsilon$ is metrically transitive, we have, by the ergodic theorem, (see [5, Chapter X, Section 2])

$$
\frac{1}{j} \sum_{t=n-j-1}^{n-1} \ln \|A+\varepsilon(t) C\| \longrightarrow \mu, \quad j \longrightarrow \infty
$$

almost surely. For any $a>1$, it follows from (i) that

$$
\sum_{j=1}^{\infty} \mathbf{P}\left[\ln ^{+}\|y(n-j-1)\|>j \ln a\right]<+\infty .
$$

Therefore,

$$
\mathbf{P}\left[\limsup _{j \rightarrow \infty}\left(\ln ^{+}\|y(n-j-1)\|>j \ln a\right)\right]=0
$$

and hence

$$
\limsup _{j \rightarrow \infty}\|(A+\varepsilon(n-1) C) \cdots(A+(n-j) C) y(n-j-1)\|^{1 / j} \leq e^{\mu}<1 .
$$

Thus, the sequence of the partial sums of series for $x(n)$ is the Cauchy sequence almost surely in $B$-norm. Hence, (see [27]) there is a random element $x(n)$ which is the limit of these sums.

By the boundedness of the operators $A$ and $C$, we find

$$
\begin{aligned}
(A+\varepsilon(n) C) x(n) & =\sum_{j=0}^{\infty}(A+\varepsilon(n) C)(A+\varepsilon(n-1) C) \cdots(A+\varepsilon(n-j) C) y(n-j-1) \\
& =x(n+1)-y(n), \quad n \in \mathbb{Z} .
\end{aligned}
$$


By Theorem 2.12, $\{x(n): n \in \mathbb{Z}\}$ is stationary. In the same manner we get the uniqueness.

Analogous to Theorem 3.14, this result can be proved for $p$-periodic processes. Instead, we give the following theorem with the moment conditions.

THEOrem 3.15 (see [9]). Let operators $\{A, C\} \subset \mathscr{L}(B)$. Suppose that $\{\varepsilon(n): n \in \mathbb{Z}\}$ is a sequence of independent identically distributed random variables in $\mathbb{C},\{y(n): n \in \mathbb{Z}\}$ is a p-periodic in distribution random process in $B$ which is independent from $\{\varepsilon(n)$ : $n \in \mathbb{Z}\}$. Let

(i) $\mathrm{E}\|y(k)\|<+\infty, 1 \leq k \leq p$;

(ii) $\mathrm{E}\|A+\varepsilon(0) C\|<1$.

Then there is a unique $p$-periodic in distribution process $\{x(n): n \in \mathbb{Z}\}$ in $B$ that satisfies (3.113) such that

$$
\mathbf{E}\|x(k)\|<+\infty, \quad 1 \leq k \leq p .
$$

Proof. Since, for every $n \in \mathbb{Z}, j \geq 1$,

$$
\begin{aligned}
\mathbf{E} \|(A+\varepsilon(n-1) C) \cdots & (A+\varepsilon(n-j) C) y(n-j-1) \| \\
& \leq(\mathbf{E}\|A+\varepsilon(0) C\|)^{j} \max _{1 \leq k \leq p} \mathbf{E}\|y(k)\|,
\end{aligned}
$$

the series

$$
x(n):=y(n-1)+\sum_{j=1}^{\infty}(A+\varepsilon(n-1) C) \cdots(A+\varepsilon(n-j) C) y(n-j-1)
$$

converges almost surely in $B$-norm and

$$
\mathbf{E}\|x(k)\|<+\infty, \quad 1 \leq k \leq p .
$$

By Theorem 2.12, the process $\{x(n): n \in \mathbb{Z}\}$ is $p$-periodic in distribution.

3.3. Nonlinear equation. In this section, we consider some nonlinear equations, which are disturbed by stochastic processes, and give conditions for existence stationary or periodic in distribution solutions to these equations.

3.3.1. A nonlinearity satisfying Lipschitz condition. Let $p \in \mathbb{N}$ be given.

THEOREM 3.16 (see [15]). Let $G: \mathbb{Z} \times B \rightarrow B$ be a function such that

(i) $G(n+p, x)=G(n, x), x \in B, n \in \mathbb{N}$;

(ii) there is $\lambda \in[0,1):\|G(n, x)-G(n, y)\| \leq \lambda\|x-y\|,\{x, y\} \subset B$;

(iii) there is $\mu \in[0,1):\|G(n, x)\| \leq \mu(1+\|x\|)$ for all $n \in \mathbb{Z},\{x, y\} \subset B$.

Let $\{y(n): n \in \mathbb{Z}\}$ be a p-periodic in distribution process in $B$ such that

$$
\mathbf{E}\|y(k)\|<+\infty, \quad 1 \leq k \leq p .
$$

Then the equation

$$
x(n+1)=G(n, x(n))+y(n), \quad n \in \mathbb{Z}
$$


has a unique $p$-periodic in distribution solution $\{x(n): n \in \mathbb{Z}\}$ satisfying

$$
\mathbf{E}\|x(k)\|<+\infty, \quad 1 \leq k \leq p .
$$

Proof. Given $n_{0} \in \mathbb{Z}$ and $x_{0} \in B$, define the sequence $\left\{x\left(n ; n_{0}, x_{0}\right): n \geq n_{0}\right\}$ by

$$
x\left(n_{0} ; n_{0}, x_{0}\right):=x_{0} ; \quad x\left(n+1 ; n_{0}, x_{0}\right):=G\left(n, x\left(n ; n_{o}, x_{0}\right)\right)+y(n), \quad n \geq n_{0} .
$$

Now let $n \in \mathbb{Z}$ and $x_{0}$ be fixed. To prove that the sequence $\left\{x\left(n ; n_{0}, x_{0}\right): n_{0} \leq n\right\}$ is the Cauchy sequence almost surely in $B$-norm, we obtain for $n_{1}<n_{2}<n$ the following inequality:

$$
\begin{aligned}
\left\|x\left(n ; n_{1}, x_{0}\right)-x\left(n ; n_{2}, x_{0}\right)\right\| & =\left\|x\left(n ; n_{2}, x\left(n_{2} ; n_{1}, x_{0}\right)\right)-x\left(n ; n_{2}, x_{0}\right)\right\| \\
& \leq \lambda\left\|x\left(n-1 ; n_{2}, x\left(n_{2} ; n_{1}, x_{0}\right)\right)-x\left(n-1 ; n_{2}, x_{0}\right)\right\| \\
& \leq \cdots \leq \lambda^{n-n_{2}}\left\|x\left(n_{2} ; n_{1}, x_{0}\right)-x_{0}\right\|
\end{aligned}
$$

almost surely. In addition,

$$
\begin{aligned}
\left\|x\left(n_{2} ; n_{1}, x_{0}\right)-x_{0}\right\|= & \left\|G\left(n_{2}-1, x\left(n_{2}-1 ; n_{1}, x_{0}\right)\right)+y\left(n_{2}-1\right)-x_{0}\right\| \\
\leq & \left\|x_{0}\right\|+\left\|y\left(n_{2}-1\right)\right\|+\mu+\mu\left\|x\left(n_{2}-1 ; n_{1}, x_{0}\right)\right\| \\
\leq & \left\|x_{0}\right\|+\left\|y\left(n_{2}-1\right)\right\|+\mu+\mu\left\|y\left(n_{2}-2\right)\right\| \\
& +\mu^{2}+\mu^{2}\left\|x\left(n_{2}-2 ; n_{1}, x_{0}\right)\right\| \\
\leq & \left\|x_{0}\right\|+\sum_{j=1}^{\infty} \mu^{j-1}\left\|y\left(n_{2}-j\right)\right\|+\frac{\mu}{1-\mu}+\mu^{n_{2}-n_{1}}\left\|x_{0}\right\|
\end{aligned}
$$

almost surely. The series in (3.130) converges almost surely by periodicity of the process $\{y(n): n \in \mathbb{Z}\}$ and the moment condition. By (3.130), it follows that

$$
\begin{aligned}
& \sum_{n_{2}=-\infty}^{n} \lambda^{n-n_{2}} \mathbf{E}\left(\sup _{n_{1}<n_{2}}\left\|x\left(n_{2} ; n_{1}, x_{0}\right)-x_{0}\right\|\right) \\
& \quad \leq \frac{2}{1-\lambda}\left\|x_{0}\right\|+\frac{\mu}{(1-\mu)(1-\lambda)}+\frac{1}{(1-\mu)(1-\lambda)} \max _{1 \leq k \leq p} \mathbf{E}\|y(k)\|
\end{aligned}
$$

and therefore, the series

$$
\sum_{n_{2}=-\infty}^{n} \lambda^{n-n_{2}} \sup _{n_{1}<n_{2}}\left\|x\left(n_{2} ; n_{1}, x_{0}\right)-x_{0}\right\|
$$

converges almost surely. Hence, for $n_{1}<n_{2}$,

$$
\lambda^{n-n_{2}}\left\|x\left(n_{2} ; n_{1}, x_{0}\right)-x_{0}\right\| \rightarrow 0, \quad n_{2} \longrightarrow-\infty
$$

almost surely. By (3.129), the sequence $\left\{x\left(n ; n_{0}, x_{0}\right): n_{0} \leq n\right\}$ is the Cauchy sequence. Thus there is (see [27]) a random element $x\left(n ; x_{0}\right)$ such that

$$
x\left(n ; x_{0}\right)=\lim _{n_{0} \rightarrow-\infty} x\left(n ; n_{0}, x_{0}\right)
$$

in the $B$-norm almost surely. 
Now we prove that for every $n \in \mathbb{Z}$, the element $x\left(n ; x_{0}\right)$ is independent from $x_{0}$ with probability one. Let $z_{0} \in B$. Then

$$
\begin{aligned}
& \left\|x\left(n ; n_{0}, x_{0}\right)-x\left(n ; n_{0}, z_{0}\right)\right\| \\
& \quad=\left\|G\left(n-1, x\left(n-1 ; n_{0}, x_{0}\right)\right)-G\left(n-1, x\left(n-1 ; n_{0}, z_{0}\right)\right)\right\| \\
& \quad \leq \lambda\left\|x\left(n-1 ; n_{0}, x_{0}\right)-x\left(n-1 ; n_{0}, z_{0}\right)\right\| \leq \cdots \leq \lambda^{n-n_{0}}\left\|x_{0}-z_{0}\right\|
\end{aligned}
$$

and therefore,

$$
\mathbf{P}\left[x\left(n ; x_{0}\right)=x\left(n ; z_{0}\right)\right]=1 .
$$

We, further, write $x(n)$ instead of $x\left(n ; x_{0}\right)$. Using (3.130) for $n_{2}=n, n_{1}=n_{0},(3.134)$ and Fatou's lemma, we obtain

$$
\mathbf{E}\|x(n)\|<+\infty .
$$

It follows from (3.128) and (3.134) that the process $\{x(n): n \in \mathbb{Z}\}$ satisfies (3.126).

To prove that the process $\{x(n): n \in \mathbb{Z}\}$ is periodic in distribution, we consider the following Borel set in $B^{\mathbb{Z}}$

$$
\begin{aligned}
\mathscr{D}:=\left\{\left\{u_{k}: k\right.\right. & \in \mathbb{Z}\}: \forall n \in \mathbb{Z}: \sum_{j=1}^{\infty} \mu^{j-1}|| u_{n-j} \|<+\infty \\
& \text { exists in } \left.B \text {-norm the limit } v_{n}:=\lim _{n_{0} \rightarrow-\infty} v_{n}\left(n_{0}, \overline{0}\right)\right\},
\end{aligned}
$$

where, for every $k \in \mathbb{Z}$, the element $v_{k}\left(n_{0}, \overline{0}\right)$ is defined for $n_{0} \leq k$ by

$$
v_{n_{0}}\left(n_{0}, \overline{0}\right):=\overline{0} ; \quad v_{k+1}\left(n_{0}, \overline{0}\right):=G\left(k, v_{k}\left(n_{0}, \overline{0}\right)\right)+u_{k}, \quad k \geq n_{0} .
$$

By definition of $\mathscr{D}$ for the sequence $\left\{u_{k}: k \in \mathbb{Z}\right\}$ and corresponding to it the sequence $\left\{v_{k}: k \in \mathbb{Z}\right\}$, it follows that

$$
v_{k+1}=G\left(k, v_{k}\right)+u_{k}, \quad k \in \mathbb{Z} .
$$

Let $T: \mathscr{D} \rightarrow B^{\mathbb{Z}}$ be the mapping defined by

$$
T\left(\left\{u_{k}: k \in \mathbb{Z}\right\}\right):=\left\{v_{k}: k \in \mathbb{Z}\right\}, \quad\left\{u_{k}: k \in \mathbb{Z}\right\} \in \mathscr{D} .
$$

The mapping $T$ is measurable and for mapping $\theta(p)$, we have

$$
T \theta(p)=\theta(p) T \text {. }
$$

Indeed, let for $\left\{u_{k}: k \in \mathbb{Z}\right\} \in \mathscr{D}$

$$
\left\{w_{k}: k \in \mathbb{Z}\right\}:=T \theta(p)\left\{u_{k}: k \in \mathbb{Z}\right\}, \quad\left\{\tilde{w}_{k}: k \in \mathbb{Z}\right\}:=\theta(p) T\left\{u_{k}: k \in \mathbb{Z}\right\} .
$$

Then

$$
\begin{aligned}
w_{k+1} & =G\left(k, w_{k}\right)+u_{k+p} ; \\
\tilde{w}_{k+p+1} & =G\left(k+p, \tilde{w}_{k+p}\right)+u_{k+p}
\end{aligned}
$$


for all $k \in \mathbb{Z}$. According to (ii), we have

$$
\left\|w_{k+1}-\tilde{w}_{k+p+1}\right\| \leq \lambda\left\|w_{k}-\tilde{w}_{k+p}\right\| \leq \cdots \leq \lambda^{j}\left\|w_{k-j+1}-\tilde{w}_{k+p-j+1}\right\|
$$

for $j \geq 1$. Hence by inequality, that is analogous to (3.130), it follows that

$$
w_{k+1}=\tilde{w}_{k+p+1}, \quad k \in \mathbb{Z} .
$$

By the previous argument, we have

$$
\mathbf{P}[\{y(n): n \in \mathbb{Z}\} \in \mathscr{D}]=1 .
$$

Let $\mu_{x}$ and $\mu_{y}$ be the measures on $\mathscr{B}\left(B^{\mathbb{Z}}\right)$ generating by the processes $\{x(n): n \in \mathbb{Z}\}$ and $\{y(n): n \in \mathbb{Z}\}$, respectively. By Lemma 2.4,

$$
\mu_{x}=\mu_{y} T^{-1}=\mu_{y} \theta(p)^{-1} T^{-1}=\mu_{y} T^{-1} \theta(p)^{-1}=\mu_{x} \theta(p)^{-1} .
$$

By Lemma 2.4, the process $\{x(n): n \in \mathbb{Z}\}$ is $p$-periodic in distribution.

To prove the uniqueness, we consider a $p$-periodic in distribution process $\{z(n)$ : $n \in \mathbb{Z}\}$ which is the solution of (3.126), such that $\mathbf{E}\|z(k)\|<+\infty, 1 \leq k \leq p$. Then

$$
\begin{aligned}
\mathbf{E}\|x(n)-z(n)\| & \leq \lambda \mathbf{E}\|x(n-1)-z(n-1)\| \leq \cdots \leq \lambda^{j} \mathbf{E}\|x(n-j)-z(n-j)\| \\
& \leq \lambda^{j} \max _{1 \leq j \leq p}(\mathbf{E}\|x(k)\|+\mathbf{E}\|z(k)\|), \quad j \geq 1 .
\end{aligned}
$$

Thus

$$
\mathbf{P}[x(n)=z(n), n \in \mathbb{Z}]=1 .
$$

REMARK 3.17. Given $x_{0} \in B$, the sequence $\{z(n): n \geq 0\}$ is defined by

$$
z(0):=x_{0} ; \quad z(n+1)=G(n, z(n))+y(n), \quad n \geq 0 .
$$

By the conditions of Theorem 3.16, the sequence $\{z(n): n \geq 0\}$ is asymptotically $p$-periodic, that is,

$$
\|z(n)-x(n)\| \longrightarrow 0, \quad n \rightarrow \infty
$$

almost surely, where $\{x(n): n \in \mathbb{Z}\}$ is $p$-periodic in distribution solution of (3.126).

EXAMPLE 3.18. Let $B=H=L_{2}([0,1])$ and $K:[0,1]^{2} \times \mathbb{R} \rightarrow \mathbb{R}$ be a measurable function satisfying the conditions:

$$
\begin{aligned}
& \exists \lambda \in[0,1):|K(s, t ; x)-K(s, t ; y)| \leq \lambda|x-y|, \\
& \exists \mu \in[0,1):|K(s, t ; x)| \leq \mu(1+|x|)
\end{aligned}
$$

for all $\{s, t\} \subset[0,1]^{2},\{x, y\} \subset \mathbb{R}$. Let $\{y(n): n \in \mathbb{Z}\}$ be stationary sequence in $L_{2}([0,1])$ such that

$$
\text { Ey }(n, t)=0, \quad t \in[0,1] ; n \in \mathbb{Z} .
$$

For every $f \in L_{2}([0,1])$, there is a stationary $L_{2}([0,1])$-valued sequence $\{x(n): n \in \mathbb{Z}\}$ satisfying the equation

$$
x(n+1, t)=\int_{0}^{1} K(t, s ; x(n, s)) d s+f(t)+y(n, t), \quad t \in[0,1], n \in \mathbb{Z} .
$$


This result is important for applications. By a fixed point theorem, the sequence, defined by

$$
z_{0} \in L_{2}([0,1]) ; \quad z_{n+1}(t)=\int_{0}^{1} K\left(t, s ; z_{n}(s)\right) d s+f(t), \quad t \in[0,1], n \geq 0
$$

converges in $L_{2}([0,1])$ to a unique solution of the integral equation

$$
z(t)=\int_{0}^{1} K(t, s ; z(s)) d s+f(t) \quad t \in[0,1] .
$$

If each function $z_{n+1}$ from (3.156) is defined with the random error $y(n)$ as in (3.155), and errors $\{y(n)\}$ forms a stationary process, then the sequence $\{x(n)\}$ also is stationary process and consequently does not converge to solution of (3.157). However, there exist methods for estimation of solution for (3.157) in this situation, see [4, 13].

3.3.2. Equations near to linear. We apply the techniques described above to study the existence stationary and periodic in distribution solution of nonlinear stochastic difference equations, which are near to linear equations. Of course, these conditions are only sufficient.

If $A \in \mathscr{L}(B)$ is an operator such that $\sigma(A) \cap S=\varnothing$, let $P_{-}$and $P_{+}$be the spectral projectors corresponding to the spectral sets that lie inside and outside of $S$, respectively.

THEOREM 3.19 (see [15]). Let $G: \mathbb{Z} \times B \rightarrow B$ be a function such that

(i) for all $n \in \mathbb{Z}$, for all $x \in B, G(n+p, x)=G(n, x)$;

(ii) there are an operator $A \in \mathscr{L}(B)$ and a number $L \geq 0$ such that

$$
\begin{gathered}
\sigma(A) \cap S=\varnothing ; \\
\forall k \in\{1,2, \ldots, p\}, \forall\{x, y\} \subset B:\|G(k, x)-G(k, y)-A(x-y)\| \leq L\|x-y\| ; \\
L_{1}:=L\left(\sum_{j=0}^{\infty}\left\|\left(A P_{-}\right)^{j}\right\|+\sum_{j=-\infty}^{-1}\left\|\left(A P_{+}\right)^{j}\right\|\right)<1 .
\end{gathered}
$$

Let $\{y(n): n \in \mathbb{Z}\}$ be a p-periodic in distribution process in $B$, such that

$$
\mathbf{E}\|y(k)\|<\infty, \quad 1 \leq k \leq p .
$$

Then the equation

$$
x(n+1)=G(n, x(n))+y(n), \quad n \in \mathbb{Z}
$$

has a unique p-periodic in distribution solution $\{x(n): n \in \mathbb{Z}\}$ with $\mathbf{E}\|x(k)\|<+\infty$, $1 \leq k \leq p$.

Proof. Let $x_{0} \in B$. By Theorem 3.2, there is a unique $p$-periodic in distribution random process $\left\{x_{1}(n): n \in \mathbb{Z}\right\}$ in $B$, such that

$$
\begin{aligned}
x_{1}(n+1)= & A x_{1}(n)+G\left(n, x_{0}\right)-A x_{0}+y(n), \quad n \in \mathbb{Z} ; \\
& \mathbf{E}\left\|x_{1}(k)\right\|<+\infty, \quad 1 \leq k \leq p .
\end{aligned}
$$


Given for $j \geq 1$ the $p$-periodic in distribution process $\left\{x_{j}(n): n \in \mathbb{Z}\right\}$, by Theorem 3.2 there is a unique $p$-periodic in distribution solution $\left\{x_{j+1}(n): n \in \mathbb{Z}\right\}$ of

$$
x_{j+1}(n+1)=A x_{j+1}(n)+G\left(n, x_{j}(n)\right)-A x_{j}(n)+y(n), \quad n \in \mathbb{Z},
$$

in addition, $\mathbf{E}\left\|x_{j+1}(k)\right\|<+\infty, 1 \leq k \leq p$. We also have

$$
\begin{aligned}
x_{m+1}(n)= & \sum_{j=0}^{\infty}\left(A P_{-}\right)^{j}\left(G\left(n-1-j, x_{m}(n-1-j)\right)-A x_{m}(n-1-j)+y(n-1-j)\right) \\
& -\sum_{j=-\infty}^{-1}\left(A P_{+}\right)^{j}\left(G\left(n-j-1, x_{m}(n-1-j)\right)-A x_{m}(n-1-j)+y(n-1-j)\right)
\end{aligned}
$$

for any $n \in \mathbb{Z}$ and $m \geq 1$. By (ii) of Theorem 3.19, we obtain

$$
\mathbf{E}\left\|x_{m+1}(n+1)-x_{m}(n+1)\right\| \leq L_{1} \max _{1 \leq k \leq p} \mathbf{E}\left\|x_{m}(k)-x_{m-1}(k)\right\|, \quad m \geq 1 .
$$

Therefore,

$$
\max _{1 \leq k \leq p} \mathbf{E}\left\|x_{m+1}(k)-x_{m}(k)\right\| \leq L_{1}^{m} \max _{1 \leq k \leq p} \mathbf{E}\left\|x_{1}(k)-x_{0}\right\|, \quad m \geq 1 .
$$

From (3.164) and (3.165), we have that for every $n \in \mathbb{Z}$, the sequence of the random elements $\left\{x_{m}(n): m \geq 1\right\}$ converges in the first-order mean [27] to a random element $x(n)$ with $\mathbf{E}\|x(n)\|<+\infty$. According to Theorem 2.14, $\{x(n): n \in \mathbb{Z}\}$ is $p$-periodic in distribution in $B$. In addition, from (3.162), we have (3.160).

To prove the uniqueness, we suppose that $\{z(n): n \in \mathbb{Z}\}$ is a $p$-periodic in the distribution solution of (3.160). By Theorem 3.2, we have

$$
\begin{aligned}
x(n)= & \sum_{j=0}^{\infty}\left(A P_{-}\right)^{j}(G(n-1-j, x(n-1-j))-A x(n-1-j)+y(n-1-j)) \\
& -\sum_{j=-\infty}^{-1}\left(A P_{+}\right)^{j}(G(n-1-j, x(n-1-j))-A x(n-1-j)+y(n-1-j))
\end{aligned}
$$

for any $n \in \mathbb{Z}$ and for the analogous representation of $z(n)$. Then

$$
\mathbf{E}\|x(n+1)-z(n+1)\| \leq L_{1} \max _{1 \leq k \leq p} \mathbf{E}\|x(k)-z(k)\| .
$$

Since

$$
\mathbf{E}\|x(k)-z(k)\| \leq \max _{1 \leq k \leq p}(\mathbf{E}\|x(k)\|+\mathbf{E}\|z(k)\|),
$$

we obtain

$$
\mathbf{E}\|x(n)-z(n)\|=0, \quad n \in \mathbb{Z} .
$$

Theorems like Theorem 3.19 may be proved for other difference equations. An analogous proof leads to the following results. 
THEOREM 3.20. Let $A$ be a closed linear operator with domain $D(A)$ such that

$$
\sigma(A) \cap[-4,0]=\varnothing .
$$

Suppose that a function $f: \mathbb{Z} \times B^{3} \rightarrow B$ satisfies the following conditions:

(i) $f(n+p, u, v, w)=f(n, u, v, w)$;

(ii) the following inequality holds:

$$
\left\|f\left(n, u_{1}, v_{1}, w_{1}\right)-f\left(n, u_{2}, v_{2}, w_{2}\right)\right\| \leq L\left(\left\|u_{1}-u_{2}\right\|+\left\|v_{1}-v_{2}\right\|+\left\|w_{1}-w_{2}\right\|\right)
$$

for all $n \in \mathbb{Z},\left\{u, v, w, u_{j}, v_{j}, w_{j}, j=1,2\right\} \subset B$ with a number $L$ such that

$$
L_{2}:=3 L \sum_{k \in \mathbb{Z}}\|c(k)\|<1,
$$

where $\{c(k)\}$ are the Laurent series coefficients of the function

$$
\left(A-\left(\frac{1}{z}-2+z\right) I\right)^{-1} \text {. }
$$

Let $\{y(n): n \in \mathbb{Z}\}$ be a p-periodic in distribution process such that

$$
\mathbf{E}\|y(k)\|<+\infty, \quad 1 \leq k \leq p .
$$

Then the equation

$$
A x(n)=x(n+1)-2 x(n)+x(n-1)+f(n, x(n+1), x(n), x(n-1))+y(n), \quad n \in \mathbb{Z}
$$

has a unique p-periodic in distribution solution $\{x(n): n \in \mathbb{Z}\}$ and

$$
\mathbf{P}[x(n) \in D(A), n \in \mathbb{Z}]=1, \quad \mathbf{E}\|x(k)\|<+\infty, \quad 1 \leq k \leq p .
$$

THEOREM 3.21. Let $A_{0}, A_{1}, \ldots, A_{p}$ be an operator from $\mathscr{L}(B)$ such that, for every $z \in S$, the operator

$$
A_{0} z^{p}+A_{1} z^{p-1}+\cdots+A_{p}
$$

has the bounded inverse $R(z)$. Suppose that $b: B^{2} \rightarrow B$ is a function which satisfies the conditions:

(i) there is $L_{1}>0:\|b(u, v)\| \leq L_{1}(1+\|u\|+\|v\|)$;

(ii) there is $L_{2}>0:\left\|b\left(u_{1}, v\right)-b\left(u_{2}, v\right)\right\| \leq L_{2}\left\|u_{1}-u_{2}\right\|$ for all $\left\{u, v, u_{1}, u_{2}\right\}$ and

$$
L_{2} \sum_{k \in \mathbb{Z}}\|c(k)\|<1
$$

where $\{c(k)\}$ are the Laurent series coefficients of $R$.

Let $\{y(n): n \in \mathbb{Z}\}$ be a stationary process such that

$$
\mathbf{E}\|y(0)\|<+\infty \text {. }
$$

Then the equation

$$
A_{0} x(n)+A_{1} x(n-1)+\cdots+A_{p} x(n-p)=b(x(n), y(n)), \quad n \in \mathbb{Z}
$$

has a unique stationary solution $\{x(n): n \in \mathbb{Z}\}$ such that $\mathbf{E}\|x(0)\|<+\infty$. 
3.4. Second-order stationary and periodic solutions. Let $B=H$ be a Hilbert space over $\mathbb{R}$. The existence problems of SO stationary and SO periodic in distribution solutions for difference equations are more complicated than those from previous sections. Finite-dimensional stochastic equations with constant coefficients are studied in detail, see Arató [1]. The aim of this section is to prove criteria for the existence of SO stationary and SO periodic solutions for linear stochastic difference equations in a Hilbert space [6].

3.4.1. Second-order stationary solutions. Let $x$ and $y$ be the $H$-valued random elements with $\mathbf{E}\|x\|^{2}<+\infty, \mathbf{E}\|y\|^{2}<+\infty$. If

$$
\forall\{u, v\} \subset H: \mathbf{E}[(x, u)(y, v)]=0,
$$

then $x$ and $y$ are said to be orthogonal to each other. We note that mutual correlation operator of orthogonal elements is the zero operator, and that orthogonality of mutually Gaussian elements implies their independence.

Given sequence $\{y(n): n \in \mathbb{Z}\}$ of random elements such that $\mathbf{E}\|y(n)\|^{2}<+\infty$, $n \in \mathbb{Z}$ and $m \in \mathbb{Z}$, let $\mathbf{L}_{m}^{y}$ be mean-square closure of linear combination

$$
A_{1} y\left(n_{1}\right)+A_{2} y\left(n_{2}\right)+\cdots+A_{k} y\left(n_{k}\right)
$$

where $k \geq 1,\left\{A_{1}, A_{2}, \ldots, A_{k}\right\} \subset \mathscr{L}(H), n_{j} \leq m, 1 \leq j \leq k$.

THEOREM 3.22. Let $A \in \mathscr{L}(H)$. The equation

$$
x(n+1)=A x(n)+y(n), \quad n \in \mathbb{Z}
$$

has a unique SO stationary solution $\left\{x(n) \in \mathbf{L}_{n-1}^{y}: n \in \mathbb{Z}\right\}$ for every SO stationary sequence $\{y(n): n \in \mathbb{Z}\}$ of pairwise orthogonal $H$-valued random elements, if and only if, for every orthonormal basis $\left\{e_{j}: j \geq 1\right\}$ in $H$, the inequality

$$
\sup _{j \geq 1} \sum_{k=0}^{\infty}\left\|A^{k} e_{j}\right\|^{2}<+\infty
$$

is valid.

\section{PROOF}

NeCESSITY. Let $\left\{x(n) \in \mathbf{L}_{n-1}^{y}: n \in \mathbb{Z}\right\}$ be an SO stationary solution of (3.183) corresponding to an SO stationary process $\{y(n): n \in \mathbb{Z}\}$ with a correlation operator $S_{y}$ of $y(0)$. From (3.183), we have

$$
\mathrm{E} x(0)=A \mathrm{E} x(0)+\mathbf{E} y(0)
$$

This equation has a unique solution $\mathbf{E} x(0)$ for every $\mathbf{E} y(0)$. Hence $1 \in(\mathbb{C} \backslash \sigma(A))$. It can be supposed that $\mathbf{E} y(0)=\mathbf{E} x(0)=\overline{0}$. Taking into account (3.183), we have

$$
\begin{aligned}
& \mathbf{E}[(x(n+1), u)(x(n+1), v)] \\
&= \mathbf{E}[(A x(n), u)(A x(n), v)]+\mathbf{E}[(y(n), u)(y(n), v)] \\
&+\mathbf{E}[(A x(n), u)(y(n), v)]+\mathbf{E}[(y(n), u)(A x(n), v)] .
\end{aligned}
$$


Since $x(n) \in \mathbf{L}_{n-1}^{y}$, we obtain that the correlation operator $S_{x}$ of the element $x(0)$ satisfies the equality

$$
S_{x}=A S_{x} A^{*}+S_{y}
$$

where $A^{*}$ is the adjoint operator of $A$. From (3.187), for every $n \in \mathbb{N}$, we have

$$
S_{x}=A^{n+1} S_{x} A^{*(n+1)}+\sum_{k=0}^{n} A^{k} S_{y} A^{* k} .
$$

Hence, taking into account the properties of the correlation operators, the convergence of the following series is obtained

$$
\sum_{k=0}^{\infty}\left(A^{k} S_{y} A^{* k} z, z\right), \quad z \in H
$$

By the condition of theorem, series (3.189) is convergent for an arbitrary correlation operator $S_{y}$. Thus,

$$
\forall z \in H:\left(A^{n+1} S_{x} A^{*(n+1)} z, z\right) \longrightarrow 0, \quad n \longrightarrow \infty ;
$$

and from (3.188), the following equality is obtained

$$
\left(S_{x} z, z\right)=\sum_{k=0}^{\infty}\left(A^{k} S_{y} A^{* k} z, z\right), \quad z \in H .
$$

Now let $\left\{e_{j}: j \geq 1\right\}$ be an arbitrary orthonormal basis in $H$. For every sequence of positive numbers such that

$$
\sum_{j=1}^{\infty} \lambda_{j}<+\infty
$$

the operator

$$
S_{y}:=\sum_{j=1}^{\infty} \lambda_{j}\left(\cdot, e_{j}\right) e_{j}
$$

is a correlation operator. For the corresponding operator $S_{x}$, equality (3.191) is true, which implies the following equality for the trace of operator $S_{x}$

$$
\operatorname{tr} S_{x}=\sum_{j=1}^{\infty}\left(S_{x} e_{j}, e_{j}\right)=\sum_{j=1}^{\infty} \sum_{k=0}^{\infty}\left(A^{k} S_{y} A^{* k} e_{j}, e_{j}\right)=\sum_{j=1}^{\infty} \lambda_{j}\left(\sum_{k=0}^{\infty}\left\|A^{k} e_{j}\right\|^{2}\right) .
$$

From the convergence of the last series for every sequence of positive numbers $\left\{\lambda_{j}\right.$ : $j \geq 1$ \} satisfying (3.192), it follows that

$$
\sup _{j \geq 1} \sum_{k=0}^{\infty}\left\|A^{k} e_{j}\right\|^{2}<+\infty .
$$


SUFFICIENCY. If $1 \in(\mathbb{C} \backslash \sigma(A))$, the expectation $\mathbf{E} x(0)$ is a unique solution of

$$
\mathbf{E} x(0)=A \mathbf{E} x(0)+\mathbf{E} y(0)
$$

for every $\mathbf{E} y(0)$. To prove that $1 \notin \sigma(A)$, we first note that by the condition of Theorem 3.22,

$$
\forall x \in H: \sum_{k=0}^{\infty}\left\|A^{k} x\right\|^{2}<+\infty .
$$

Therefore, on $H$ is well-defined the linear operator $\mathscr{A}: H \rightarrow l_{2}(H)$, where

$$
\begin{aligned}
& \mathscr{A} x:=\left(x, A x, A^{2} x, \ldots\right), \quad x \in H, \\
& l_{2}(H):=\left\{\left(u_{n}: n \geq 0\right) \mid u_{n} \in H, n \geq 0 ; \sum_{n=0}^{\infty}\left\|u_{n}\right\|^{2}<+\infty\right\}
\end{aligned}
$$

is a Hilbert space. The operator $\mathscr{A}$ is closed. Indeed, if $x_{j} \rightarrow x$ in $H$ and $\mathscr{A} x_{j} \rightarrow u=$ $\left(u_{n}: n \geq 0\right)$ in $l_{2}(H)$, then for every $n \geq 0$, we obtain

$$
A^{n} x_{j} \rightarrow A^{n} x, \quad A^{n} x_{j} \rightarrow u_{n}, \quad j \rightarrow \infty .
$$

Hence $u_{n}=A^{n} x, n \geq 0$ and $u=\mathscr{A} x$. By the closed graph theorem, the operator $\mathscr{A}$ is bounded and

$$
\forall x \in H: \sum_{n=0}^{\infty}\left\|A^{n} x\right\|^{2} \leq\|\mathscr{A}\|^{2}\|x\|^{2}
$$

it follows that

$$
\sum_{n=0}^{\infty}\left\|A^{n+k} x\right\|^{2} \leq\|\mathscr{A}\|^{2}\left\|A^{k} x\right\|^{2}, \quad k \geq 0 .
$$

Therefore,

$$
\sum_{k=0}^{\infty} \sum_{n=0}^{\infty}\left\|A^{n+k} x\right\|^{2}=\sum_{n=0}^{\infty}(n+1)\left\|A^{n} x\right\|^{2} \leq\|\mathscr{A}\|^{4}\|x\|^{2} .
$$

In the same way, it follows that

$$
\sum_{n=0}^{\infty}(n+1)(n+2)\left\|A^{n} x\right\|^{2} \leq 2\|\mathscr{A}\|^{6}\|x\|^{2} .
$$

By Cauchy's inequality, we obtain

$$
\sum_{n=0}^{\infty}\left\|A^{n} x\right\|=\sum_{n=0}^{\infty} \frac{1}{n}\left(n\left\|A^{n} x\right\|\right) \leq\left(\sum_{n=0}^{\infty} \frac{1}{n^{2}} \sum_{n=0}^{\infty} n^{2}\left\|A^{n} x\right\|^{2}\right)^{1 / 2}<+\infty .
$$

Thus, the operator

$$
I+A+A^{2}+\cdots+A^{n}+\cdots
$$

is bounded inverse to $I-A$. There is no loss of generality in assuming that $\mathbf{E} y(0)=\overline{0}$. 
Fix arbitrary $n \in \mathbb{Z}$. Consider the following sequence of random elements in $H$ :

$$
\xi(m):=\sum_{j=0}^{m} A^{j} y(n-1-j) \in \mathbf{L}_{n-1}^{y}, \quad m \geq 1 .
$$

The element $\xi(n)$ has the correlation operator

$$
T(m)=\sum_{j=0}^{m} A^{j} S_{y} A^{* j}, \quad m \geq 1
$$

and for $1 \leq m<k$, the following equality

$$
\mathbf{E}\|\xi(k)-\xi(m)\|^{2}=\operatorname{tr}\left(\sum_{j=m+1}^{k} A^{j} S_{y} A^{* j}\right)
$$

holds. For the orthonormal basis $\left\{e_{j}: j \geq 1\right\}$ in $H$ consisting of the eigenvectors of the operator $S_{y}$, we obtain, from representation (3.208),

$$
\mathbf{E}\|\xi(k)-\xi(m)\|^{2}=\sum_{t=1}^{\infty} \lambda_{t} \sum_{j=m+1}^{k}\left\|A^{j} e_{t}\right\|^{2},
$$

where $\left\{\lambda_{j}: j \geq 1\right\}$ are the eigenvalues of operator $S_{y}$ corresponding to the eigenvectors $\left\{e_{j}: j \geq 1\right\}$. By the conditions of Theorem 3.22, for every $t \geq 1$,

$$
\sum_{j=m+1}^{\infty}\left\|A^{j} e_{t}\right\|^{2} \longrightarrow 0, \quad m \rightarrow \infty
$$

Furthermore, for $m \geq 1$,

$$
\sum_{j=m+1}^{\infty}\left\|A^{j} e_{t}\right\|^{2} \leq \sup _{t \geq 1} \sum_{j=0}^{\infty}\left\|A^{j} e_{t}\right\|^{2}<+\infty .
$$

Hence, the sequence $\{\xi(m): m \geq 1\}$ is a Cauchy sequence in the quadratic mean; and there is a random element $x(n)$ such that

$$
\mathbf{E}\|x(n)\|^{2}<+\infty, \quad x(n)=\sum_{j=0}^{\infty} A^{j} y(n-j-1) \in \mathbf{L}_{n-1}^{y},
$$

see [27]. The last series is convergent in the quadratic mean, with the correlation operator $S_{x}$ of the element $x(0)$ being

$$
S_{x}=\sum_{j=0}^{\infty} A^{j} S_{y} A^{* j},
$$

the series in (3.213) converging in the trace norm. Also, for every $n \in \mathbb{Z}, \mathbf{E} x(n)=\overline{0}$, the mutual correlation operator $S_{k, n}$ of the elements $x(k)$ and $x(n)$ has the following 
representation:

$$
\left(S_{k, n} u, v\right)=\sum_{j=0}^{\infty}\left(A^{n-k+j} S_{y} A^{* j} u, v\right), \quad\{u, v\} \subset H .
$$

Hence,

$$
S_{k, n}=A^{n-k} S_{x}, \quad\{k, n\} \subset \mathbb{Z}, k \leq n .
$$

Thus, the process $\{x(n): n \in \mathbb{Z}\}$ is SO stationary in $H$. Moreover, since $A \in \mathscr{L}(H)$ with probability one,

$$
A x(n)+y(n)=\sum_{j=0}^{\infty} A^{j+1} y(n-j-1)+y(n)=x(n+1),
$$

for every $n \in \mathbb{Z}$.

To complete the proof of sufficiency, we need only to show uniqueness. Let $\{z(n)$ : $n \in \mathbb{Z}\}$ be an SO stationary solution of (3.183) satisfying the conditions of Theorem 3.22. Then for every $n \in \mathbb{Z}$, we have

$$
x(n)-z(n)=A^{n-m}(x(m)-z(m)), \quad m \leq n-1 .
$$

It is easy to see that

$$
\begin{aligned}
\mathbf{E}\|x(n)-z(n)\|^{2} & \leq 2 \mathbf{E}\left\|A^{n-m} x(m)\right\|^{2}+2 \mathbf{E}\left\|A^{n-m} z(m)\right\|^{2} \\
& =2 \operatorname{tr}\left(A^{n-m} S_{x} A^{*(n-m)}\right)+2 \operatorname{tr}\left(A^{n-m} S_{z} A^{*(n-m)}\right),
\end{aligned}
$$

where $S_{z}$ is the correlation operator of $z(0)$. By (3.191) we have $S_{x}=S_{z}$. Combining (3.188) and (3.191), we claim that

$$
\begin{aligned}
\operatorname{tr}\left(A^{n-m} S_{x} A^{*(n-m)}\right) & =\operatorname{tr}\left(\sum_{j=n-m}^{\infty} A^{j} S_{y} A^{* j}\right) \\
& =\sum_{t=1}^{\infty} \lambda_{t} \sum_{j=n-m}^{\infty}\left\|A^{j} e_{t}\right\|^{2}, \quad m \leq n-1,
\end{aligned}
$$

where $\lambda_{t}, t \geq 1$ and $e_{t}, t \geq 1$ are the eigenvalues and eigenvectors of operator $S_{y}$ such that $e_{t}, t \geq 1$ is orthonormal basis in $H$. By the conditions of Theorem 3.22, we infer that

$$
\sum_{t=1}^{\infty} \lambda_{t} \sum_{j=n-m}^{\infty}\left\|A^{j} e_{t}\right\|^{2} \longrightarrow 0, \quad m \longrightarrow-\infty,
$$

and that $x(n)=z(n)$ with probability one for each $n \in \mathbb{Z}$.

COROLlARY 3.23. Let $\{y(n): n \in \mathbb{Z}\}$ be a sequence of Gaussian independent identically distributed random elements in $H$ with expectation $\overline{0}$ and correlation operator $S_{y}$. Let the operator $A \in \mathscr{L}(H)$ satisfy the conditions of Theorem 3.22. 
Then there is a unique stationary Gaussian Markov process $\left\{x(n) \in \mathbf{L}_{n-1}^{y}: n \in \mathbb{Z}\right\}$ satisfying (3.183).

Proof. It is a direct consequence of the previous theorem and the known properties of the Gaussian random elements, see [38, 9] for the full proof.

REMARKS. (1) We mention simple sufficient conditions ensuring the conditions of Theorem 3.22. If the spectral radius of the operator $A$ is

$$
r(A):=\lim _{n \rightarrow \infty}\left\|A^{n}\right\|^{1 / n}<1,
$$

then the conditions of Theorem 3.22 are satisfied. Indeed,

$$
\sup _{j \geq 1} \sum_{k=0}^{\infty}\left\|A^{k} e_{j}\right\|^{2} \leq \sum_{k=0}^{\infty}\left\|A^{k}\right\|^{2}
$$

with the series in the right converging by the Cauchy's theorem.

(2) If $\|A\|<1$ then conditions of Theorem 3.22 are satisfied.

(3) If $H$ is the finite-dimensional space then every stationary Gaussian Markov process satisfies an equation such as (3.183), see [1, Chapter 2, Section 2.1] and [30]. For the infinite-dimensional space, this is not true, see [9, Chapter 2, Section 2.7] for the details.

COROLLARY 3.24. Let the conditions of Theorem 3.22 be satisfied and $\mathbf{E} y(0)=\overline{0}$. For $z_{0} \in \mathbf{L}_{-1}^{y}$, the sequence $\{z(n) ; n \in \mathbb{Z}\}$ is defined by

$$
\begin{aligned}
z(n+1) & :=A z(n)+y(n), \quad n \geq 0 ; \\
z(0) & :=z_{0} .
\end{aligned}
$$

Then

$$
\mathrm{E}\|z(n)-x(n)\|^{2} \longrightarrow 0, \quad n \rightarrow \infty
$$

where $\left\{x(n) \in \mathbf{L}_{n-1}^{y}: n \in \mathbb{Z}\right\}$ is a unique SO stationary solution to (3.183).

Proof. The proof follows the lines of the proof of Theorem 3.22 and is omitted.

Corollary 3.25. Let $A \in \mathscr{L}(H)$ be such that

$$
\sup _{j \geq 1} \sum_{k=0}^{\infty}\left\|A^{k} e_{j}\right\|^{2}<+\infty
$$

for every orthonormal basis $\left\{e_{j}: j \geq 1\right\}$ in $H$.

Then the equation

$$
S_{x}=A S_{x} A^{*}+S_{y}
$$

has, in the class of all correlation operators, a unique solution $S_{x}$ for every correlation operator $S_{y}$. 
Proof. See the proof of Theorem 3.22.

3.4.2. Second-order periodic solutions. Let $p \in \mathbb{N}$ be a natural number and let $\{A(n): n \in \mathbb{Z}\}$ be the bounded linear operators such that

$$
\forall n \in \mathbb{Z}: A(n+p)=A(n) .
$$

Set

$$
B_{0}:=A(p-1) A(p-2) \cdots A(1) A(0) .
$$

THEOREM 3.26 (see [6]). The equation

$$
x(n+1)=A(n) x(n)+y(n), \quad n \in \mathbb{Z}
$$

has a unique SO $p$-periodic solution $\left\{x(n) \in \mathbf{L}_{n-1}^{y}: n \in \mathbb{Z}\right\}$ for every SO p-periodic process $\{y(n): n \in \mathbb{Z}\}$ with orthogonal values and with $\mathrm{E} y(n)=\overline{0}, n \in \mathbb{Z}$, if and only if, for every orthonormal basis $\left\{e_{j}: j \geq 1\right\}$ in $H$, the following inequality holds

$$
\sup _{j \geq 1} \sum_{k=0}^{\infty}\left\|B_{0}^{k} e_{j}\right\|^{2}<+\infty .
$$

\section{PROOF}

SUFFICIENCY. Let $p>1$. Suppose that condition (3.230) holds. Let $\{y(n): n \in \mathbb{Z}\}$, $\mathrm{E} y(n)=\overline{0}, n \in \mathbb{Z}$, be an SO $p$-periodic process with orthogonal values. Now define

$$
\begin{aligned}
\xi(k) & :=\sum_{t=0}^{p-1} C_{t} y((k+1) p-1-t), \quad k \in \mathbb{Z} ; \\
C_{0} & :=I, \quad C_{t}:=A(p-1) \cdots A(p-t), \quad 1 \leq t \leq p-1 .
\end{aligned}
$$

The elements $\{\xi(k): k \in \mathbb{Z}\}$ are pairwise orthogonal. They have expectation $\overline{0}$ and the same correlation operator

$$
\sum_{t=0}^{p-1} C_{t} S_{y,-t-1} C_{t}^{*},
$$

where $S_{y,-k}:=S_{y, k}, 0 \leq k \leq p-1$, and $S_{y, 0}, S_{y, 1}, \ldots, S_{y, p-1}$ are the correlation operators of the elements $y(0), y(1), \ldots, y(p-1)$, respectively. For every SO $p$-periodic sequence $\{u(k): k \in \mathbb{Z}\}, \mathbf{E} u(k)=\overline{0}, k \in \mathbb{Z}$, of pairwise orthogonal $H$-valued random elements, an SO $p$-periodic process $\{y(n): n \in \mathbb{Z}\}$ with orthogonal values and with $\mathrm{E} y(n)=\overline{0}, n \in \mathbb{Z}$, can be chosen in such a way that $\xi(k)=u(k), k \in \mathbb{Z}$.

By Theorem 3.22, there is a unique SO stationary solution $\left\{x_{0}(k) \in \mathbf{L}_{k-1}^{\xi}: k \in \mathbb{Z}\right\}$ of the following equation:

$$
x_{0}(k+1)=B_{0} x_{0}(k)+\xi(k), \quad k \in \mathbb{Z},
$$

and this solution has the following representation:

$$
x_{0}(k)=\sum_{j=0}^{\infty} B_{0}^{j} \xi(k-j-1), \quad k \in \mathbb{Z} .
$$


The series for $x_{0}(k)$ converges in the quadratic mean. Now define the process $\{x(n)$ : $n \in \mathbb{Z}\}$ in $H$ in the following way:

$$
\begin{aligned}
x(k p) & :=x_{0}(k), \\
x(k p+1) & :=A(k p) x(k p)+y(k p)=A(0) x_{0}(k)+y(k p), \\
x(k p+2):= & A(k p+1) x(k p+1)+y(k p+1), \\
= & A(1) A(0) x_{0}(k)+A(1) y(k p)+y(k p+1), \\
& \vdots \\
x(k p+j):= & A(k p+j-1) x(k p+j-1)+y(k p+j-1) \\
= & A(j-1) A(j-2) \cdots A(0) x_{0}(k) \\
& +\sum_{l=0}^{j-2} A(j-1) \cdots A(l+1) y(k p+l)+y(k p+j-1), \quad 2 \leq j \leq p-1 .
\end{aligned}
$$

Clearly, $x(n) \in \mathbf{L}_{n-1}^{y}$. We now show that, for each $0 \leq j \leq p-1$, the process $\{x(k p+j)$ : $k \in \mathbb{Z}\}$ is SO stationary; and by Lemma 2.5 the process $\{x(n): n \in \mathbb{Z}\}$ is SO $p$-periodic. This process satisfies (3.229). For the proof, it suffices to prove the equality

$$
x(k p+p)=A(k p+p-1) x(k p+p-1)+y(k p+p-1), \quad k \in \mathbb{Z} .
$$

For fixed $k$, we have

$$
\begin{aligned}
A(k p+ & p-1) x(k p+p-1)+y(k p+p-1) \\
= & A(p-1) A(p-2) \cdots A(0) x_{0}(k) \\
& +\sum_{j=0}^{p-3} A(p-1) \cdots A(j+1) y(k p+j) \\
& +A(p-1) y(k p+p-2)+y(k p+p-1) \\
= & B_{0} x_{0}(k)+\xi(k)=x_{0}(k+1)=x(k p+p) .
\end{aligned}
$$

The process $\{x(n): n \in \mathbb{Z}\}$ is a unique solution for (3.229). Indeed, let $\{z(n) \in$ $\left.\mathbf{L}_{n-1}^{y}: n \in \mathbb{Z}\right\}$ be an SO $p$-periodic solution for (3.229). Then

$$
\begin{aligned}
x((k+1) p) & =B x(k p)+\xi(k), \\
z((k+1) p) & =B z(k p)+\xi(k), \quad k \in \mathbb{Z}, \\
x(k p)-z(k p) & =B(x((k-1) p)-z((k-1) k p)) \\
& =B^{k-m}(x(m p)-z(m p)), \quad m \leq k-1 .
\end{aligned}
$$

Thus

$$
\begin{aligned}
\mathbf{E}\|x(k p)-z(k p)\|^{2} & \leq 2 \mathbf{E}\left\|B^{k-m} x(m)\right\|^{2}+2 \mathbf{E}\left\|B^{k-m} z(m)\right\|^{2} \\
& =2 \operatorname{tr}\left(B^{k-m} S_{x} B^{*(k-m)}\right)+2 \operatorname{tr}\left(B^{k-m} S_{z} B^{*(k-m)}\right),
\end{aligned}
$$

and as in the proof of Theorem 3.22, we have $\mathbf{P}[x(k p)=z(k p), k \in \mathbb{Z}]=1$. It follows that $\mathbf{P}[x(n)=z(n), n \in \mathbb{Z}]=1$. 
NeCESSITY. Let $\left\{x(n) \in \mathbf{L}_{n-1}^{y},: n \in \mathbb{Z}\right\}$ be a unique SO $p$-periodic solution to (3.229) for arbitrary but fixed SO $p$-periodic process $\{y(n): n \in \mathbb{Z}\}$ with orthogonal values and with $\mathrm{E} y(n)=\overline{0}, n \in \mathbb{Z}$.

It is easy to verify that, for an SO $p$-periodic process $\{x(n): n \in \mathbb{Z}\}$, for every $j, 0 \leq j \leq p-1$, the process

$$
\{x(k p+j): k \in \mathbb{Z}\}
$$

is SO stationary. Moreover, if the process $\{x(n): n \in \mathbb{Z}\}$ satisfies (3.229), then

$$
x((k+1) p)=B_{0} x(k p)+\xi(k), \quad k \in \mathbb{Z} .
$$

Let $x_{0}(k):=\mathbf{E}(x(k p) / \xi(j), j \leq k-1), k \in \mathbb{Z}$. Then $x_{0}(k) \in \mathbf{L}_{k-1}^{\xi}, k \in \mathbb{Z}$, and from (3.241), we have

$$
x_{0}(k+1)=B_{0} x_{0}(k)+\xi(k), \quad k \in \mathbb{Z} .
$$

The process $\left\{x_{0}(k): k \in \mathbb{Z}\right\}$ is a unique solution for (3.242). Indeed, if (3.242) had two different solutions, then we can construct two different solutions to (3.229) by repeating the arguments from the proof of sufficiency.

Now applying Theorem 3.22 to (3.242), we find that

$$
\sup _{j \geq 1} \sum_{k=0}^{\infty}\left\|B_{0}^{k} e_{j}\right\|^{2}<+\infty
$$

for each orthonormal basis in $H$.

COROLlary 3.27. Let the operators $\{A(n)\}$ satisfy the conditions of Theorem 3.26, $\{y(n): n \in \mathbb{Z}\}$ be a Gaussian $p$-periodic process in $H$ with independent values, and $\mathrm{E} y(n)=\overline{0}, n \in \mathbb{Z}$. Then there is a unique p-periodic Gaussian Markov process $x(n) \in$ $\mathbf{L}_{n-1}^{y}, n \in \mathbb{Z}$, satisfying (3.229).

Theorem 3.26 admits the following generalization. Let $p \in \mathbb{N} \cup\{0\}$ and let $\mathscr{P}$ be the class of all $H$-valued SO $p$-periodic processes $\{y(n): n \in \mathbb{Z}\}$, such that $\mathbf{E} y(n)=\overline{0}$, $n \in \mathbb{Z}$; the random elements $y(k)$ and $y(n)$ are orthogonal if $|k-n|>p$.

THEOREM 3.28. Let $\{A(n)\}$ be the operators from Theorem 3.26. Equation (3.229) has a unique SO p-periodic solution $\left\{x(n) \in \mathbf{L}_{n-1}^{y}: n \in \mathbb{Z}\right\}$ for every process $\{y(n)$ : $n \in \mathbb{Z}\} \in \mathscr{P}$ if and only if, for every orthonormal basis $\left\{e_{j}: j \geq 1\right\}$ in $H$, inequality (3.230) holds.

Proof. The proof is similar to that of Theorem 3.26 and so is omitted.

The following sufficient condition will be useful in the applications.

Condition 3.29. Let

$$
A(n+p)=A(n), \quad n \in \mathbb{Z} ; \quad B_{0}=A(p-1) \cdots A(0) .
$$

Suppose that the spectrum of operator $B_{0}$ consists of two parts $\sigma_{-}$and $\sigma_{+}$, such that

$$
\sup \left(|z|: z \in \sigma_{-}\right)<1, \quad \inf \left(|z|: z \in \sigma_{+}\right)>1 .
$$


THEOREM 3.30. By Condition 3.29, equation (3.229) has a unique SO p-periodic solution $\{x(n): n \in \mathbb{Z}\}$ for every SO p-periodic process $\{y(n): n \in \mathbb{Z}\}$ in $H$ with $\mathbf{E} y(k)=\overline{0}$, $1 \leq k \leq p$.

Proof. See the proof of Theorems 3.1, 3.26, and see [6] for more details.

ACKNOWLEDGMENT. The author expresses his gratitude to the referees for careful reading and helpful suggestions.

\section{REFERENCES}

[1] M. Arató, Linear Stochastic Systems with Constant Coefficients. A Statistical Approach, Lecture Notes in Control and Information Sciences, vol. 45, Springer-Verlag, Berlin, 1982.

[2] A. Araujo and E. Giné, The Central Limit Theorem for Real and Banach Valued Random Variables, Wiley Series in Probability and Mathematical Statistics, John Wiley \& sons, New York, 1980.

[3] P. Bougerol and N. Picard, Strict stationarity of generalized autoregressive processes, Ann. Probab. 20 (1992), no. 4, 1714-1730.

[4] N. A. Denisevskil and A. Y. Dorogovtsev, On the law of large numbers for a linear process in a Banach space, Soviet Math. Dokl. 36 (1988), no. 1, 47-50, [translated from Dokl. Akad. Nauk SSSR 295 (1987), no. 2, 276-279].

[5] J. L. Doob, Stochastic Processes, John Wiley \& sons, New York, 1953.

[6] A. Y. Dorogovtsev, Necessary and sufficient conditions for existence of stationary and periodic solutions of a stochastic difference equation in Hilbert space, Comput. Math. Appl. 19 (1990), no. 1, 31-37.

[7] - Stationary and periodic solutions of a stochastic difference equation in a Banach space, Theory Probab. Math. Statist. 42 (1991), 39-46, [translated from Teor. Veroyatnost. i Mat. Statist. 42 (1990), 35-42].

[8] Stationary and periodic solutions of stochastic difference and differential equations in Banach space, New Trends in Probability and Statistics, Vol. 1 (Bakuriani, 1990), VSP, Utrecht, 1991, pp. 375-390.

[9] _ Periodicheskie i Statsionarnye Rezhimy Beskonechnomernykh Determinirovannykh i Stokhasticheskikh Dinamicheskikh Sistem, Vishcha Shkola, Kiev, 1992 (Russian).

[10] _ On stability for bounded and stationary solutions of equations with operator coefficients under perturbations of coefficients, Uspehi Mat. Nauk 50 (1995), no. 4, 103.

[11] Stability of bounded and stationary solutions of linear equations with respect to perturbations of operator coefficients, Dokl. Akad. Nauk 345 (1995), no. 4, 448-450 (Russian).

[12] _ Stability of stationary and periodic solutions equations in Banach space, J. Appl. Math. Stochastic Anal. 10 (1997), no. 3, 249-255.

[13] A. Y. Dorogovtsev and N. A. Denisevskiū, Successive approximations in models with errors, Mathematics Today '93, No. 8, Vishcha Shkola, Kiev, 1993, pp. 63-77 (Russian).

[14] A. Y. Dorogovtsev and O. A. Lagoda, On bounded solutions of a difference equation in a Banach space, Mathematics Today '95, No. 10, Nauchn. Izdat. “TViMS”, Kiev, 1995, pp. 55-59 (Russian).

[15] A. Y. Dorogovtsev and V. T. Le, The existence of periodic and stationary regimes of discrete dynamical systems in a Banach space, Kibernetika (Kiev) (1988), no. 6, 121-123 (Russian).

[16] N. Dunford and J. T. Schwartz, Linear Operators. I. General Theory, Interscience Publishers, New York, 1958.

[17] I. I. Gikhman and A. V. Skorokhod, Teoriya Sluchainykh Protsessov. Tom I [Theory of Random Processes], Izdat. Nauka, Moscow, 1971 (Russian). 
[18] E. G. Gladyšev, Periodically correlated random sequences, Dokl. Akad. Nauk SSSR 137 (1961), 1026-1029 (Russian).

[19] M. F. Gorodnil and A. Y. Dorogovtsev, Stationary solutions of a two-dimensional stochastic difference equation in a Banach space, Stochastic Analysis and Its Applications, Akad. Nauk Ukrain. SSR Inst. Mat., Kiev, 1989, pp. 25-33 (Russian).

[20] _ Bounded solutions of a class of nonlinear operator difference equations, Ukrainian Math. J. 47 (1995), no. 7, 1022-1029, [translated from Ukrain. Mat. Zh. 47 (1995), no. 7, 890-896].

[21] A. Halanay, T. Morozan, and C. Tudor, Tracking discrete almost periodic signals under white noise perturbations, Internat. J. Control 47 (1988), 381-392.

[22] P. R. Halmos, Measure Theory, D. Van Nostrand, New York, 1950.

[23] E. Hille and R. S. Phillips, Functional Analysis and Semi-groups, American Mathematical Society, Rhode Island, 1957.

[24] A. Jiri, Statistical analysis of periodic autoregression, Apl. Mat. 28 (1983), no. 5, 363-385.

[25] _ On multiple periodic autoregression, Apl. Mat. 32 (1987), no. 1, 63-79.

[26] J. P. Keener, Propagation and its failure in coupled systems of discrete excitable cells, SIAM J. Appl. Math. 47 (1987), no. 3, 556-572.

[27] V. M. Kruglov, Dopolnitelnye Glavy Teorii Veroyatnostei [Supplementary Chapters of Probability Theory], Vyssh. Shkola, Moscow, 1984 (Russian).

[28] H. H. Kuo, Gaussian Measures in Banach Spaces, Springer-Verlag, Berlin, 1975.

[29] K. Kuratowski, Topology. Vol. I, Academic Press, New York, 1966.

[30] J. Lamperti, Stochastic Processes. A Survey of the Mathematical Theory, Applied Mathematical Sciences, vol. 23, Springer-Verlag, New York, 1977.

[31] A. Makagon and H. Salehi, Notes on infinite-dimensional stationary sequences, Probability Theory on Vector Spaces, IV (Łańcut, 1987) (S. Cambanis and A. Weron, eds.), Springer, Berlin, 1989, pp. 200-238.

[32] Y. Morita, A periodic wave and its stability to a circular chain of weakly coupled oscillators, SIAM J. Math. Anal. 18 (1987), 1681-1698.

[33] T. Morozan, Bounded, periodic and almost periodic solutions of affine stochastic discretetime systems, Rev. Roumaine Math. Pures Appl. 32 (1987), no. 8, 711-718.

[34] T. Mourid, Processus autorégressifs banachiques d'ordre supérieur [Banach-valued autoregressive processes ], C. R. Acad. Sci. Paris Sér. I Math. 317 (1993), 1167-1172 (French).

[35] M. O. Otelbaev, Coercive estimates for the solutions of difference equations, Trudy Mat. Inst. Steklov. 181 (1988), 241-249 (Russian).

[36] M. Pagano, On periodic and multiple autoregressions, Ann. Statist. 6 (1978), no. 6, 13101317.

[37] J. Riordan, Combinatorial Identities, John Wiley \& sons, New York, 1968.

[38] A. N. Shiryaev, Probability, Nauka, Moscow, 1980.

[39] A. V. Skorokhod, Integration in a Hilbert Space, Nauka, Moscow, 1975.

[40] B. M. Troutman, Some results in periodic autoregression, Biometrika 66 (1979), no. 2, 219-228.

A. YA. Dorogovtsev: LePSE BouleVARD, 79-A, 7 KIEV-126, 03126, UKRAINE

E-mail address: adoro@math.naverex.kiev.ua 


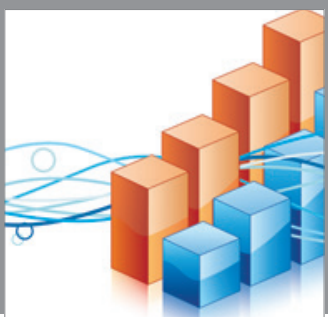

Advances in

Operations Research

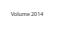

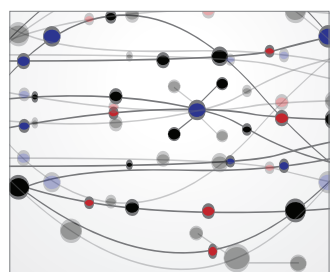

\section{The Scientific} World Journal
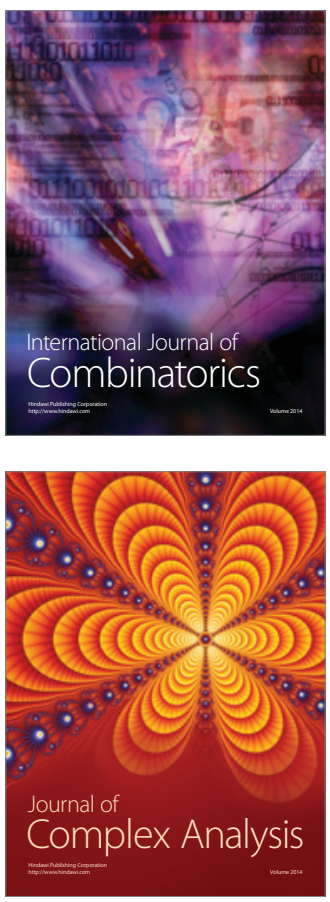

International Journal of

Mathematics and

Mathematical

Sciences
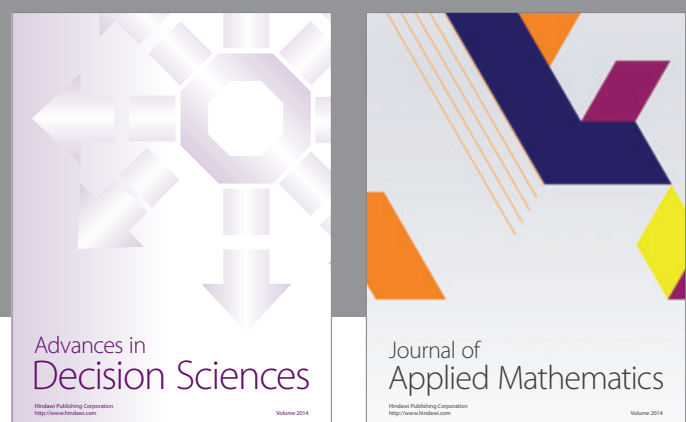

Journal of

Applied Mathematics
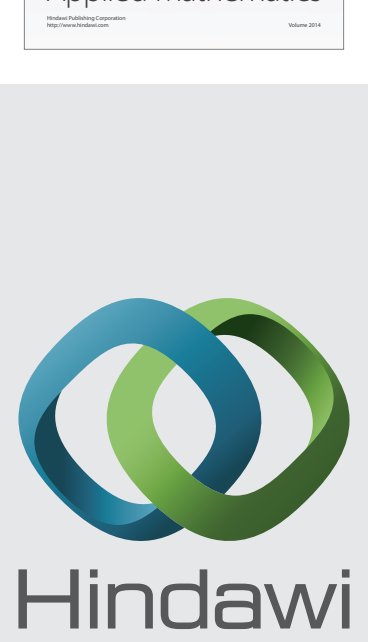

Submit your manuscripts at http://www.hindawi.com
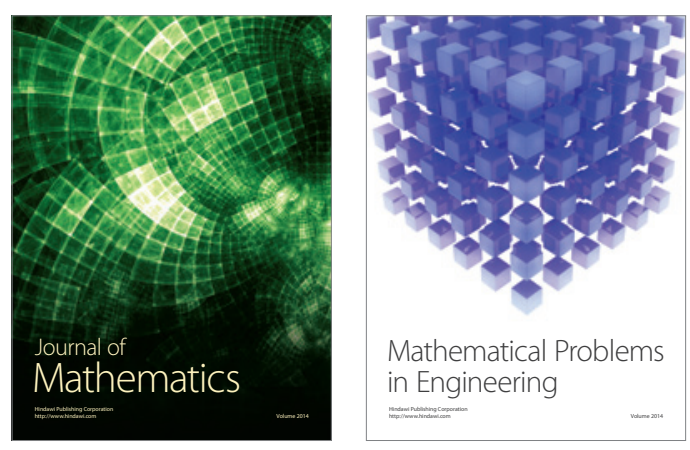

Mathematical Problems in Engineering
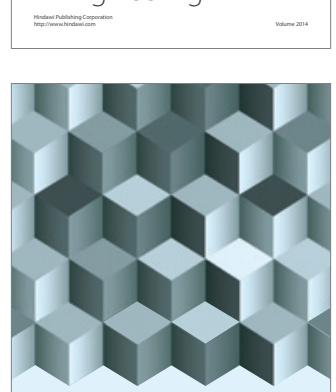

Journal of

Function Spaces
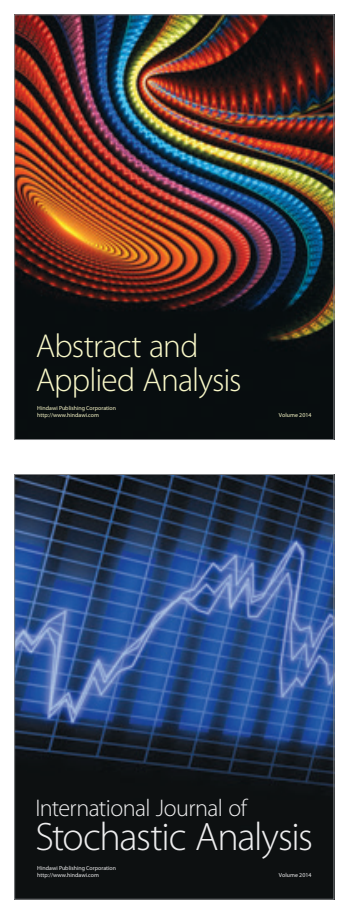

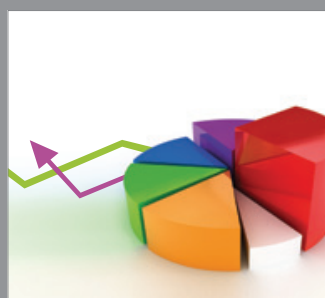

ournal of

Probability and Statistics

Promensencen
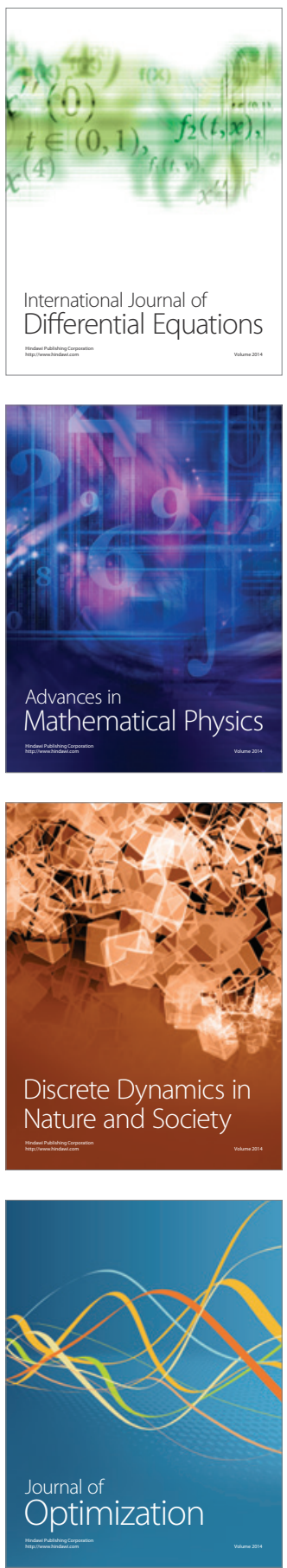